\title{
Design of Reliable and Efficient Flip-Flops for Subthreshold Operation using Multi-Threshold MOSFETs and Transistor Sizing Technique
}

by

\section{Farideh Shiran}

Thesis submitted to the Faculty of Graduate and Postdoctoral Affairs in partial fulfillment of the requirements for the degree of

\section{Master of Applied Science}

\author{
in \\ Electrical and Computer Engineering
}

Ottawa-Carleton Institute for Electrical and Computer Engineering

\author{
Carleton University \\ Ottawa, Ontario, Canada \\ (C) 2015, Farideh Shiran
}


This thesis addresses the challenge of designing reliable and energy-efficient subthreshold flip-flops. A dual-clock-phase flip-flop (TG-FF) and a single-clock-phase flip-flop (SPFF) configurations are considered and their gradual design modifications are reported towards perfecting their yields at minimum cost in terms of energy consumption. In addition, the two optimized flip-flop designs are compared based on the criteria of static and dynamic energy consumptions, delay, and reliability in face of PVT variations.

A $65 \mathrm{~nm}$ and a newer $28 \mathrm{~nm}$ CMOS technology kit were used during the course of this work. Multi-threshold MOSFETs and transistor sizing technique were incorporated for yield optimization. In general we were able to increase the flip-flops reliabilities to $100 \%$ at the nominal temperature and power supply condition, and in the worst-case around $90 \%$ in the extreme cold and $-10 \%$ noise on the power supply. We also prove the overall superiority of the SP-FF compared to TG-FF. 


\section{Acknowledgment}

First and foremost, I would like to express my deep and sincere gratitude to my supervisor Professor Maitham Shams for the continuous support, understanding, kindness, and great guidance. During my studies, he not only helped me in my research but also he taught me in my life.

I would like to thank Professor Niall Tait, Len MacEachern, and Voicu Groza for their careful review and contributions to the thesis.

I would like to thank the staff members at the Department of Electronics, especially, Blazenka Power, Anna Lee, Nagui Mikhail and Scott Bruce.

I would also like to thank my friends Mohammadjavad Sheikhzadeh Nadjar, Sukneet Basuta, Behzad Yadegari, and Roya Dibaj for their technical and moral support.

Last but not least, I owe my deepest gratitude to my mother, who gave me the feelings of warmth, contentment and love; And to my lovely families, for always being there for me. 


\section{Dedication}

This dissertation is dedicated to...

my father's soul,

for believing in me and for his support and encouragement. 


\section{Table of Contents}

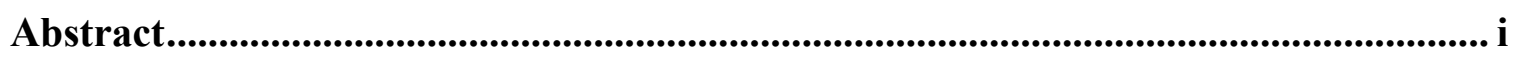

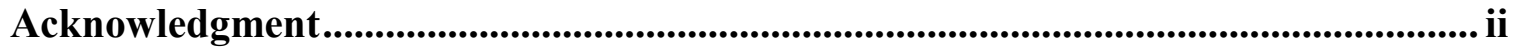

Table of Contents ....................................................................................................................... iv

List of Figures.............................................................................................................................. vii

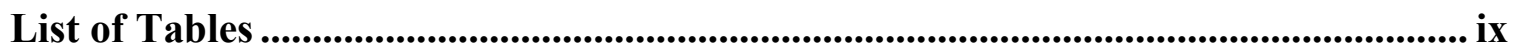

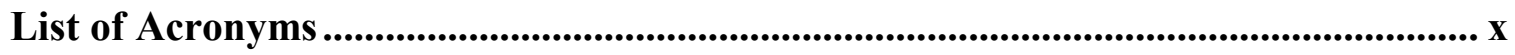

Chapter 1. Introduction ............................................................................................................... 1

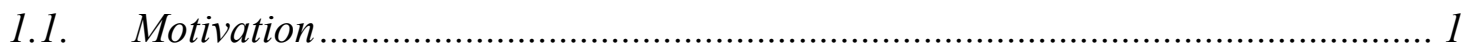

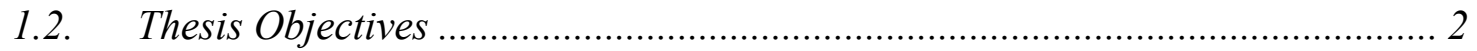

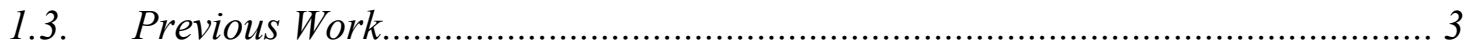

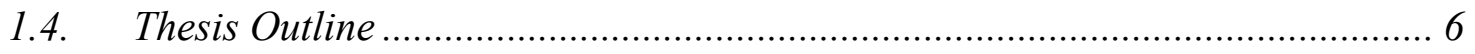

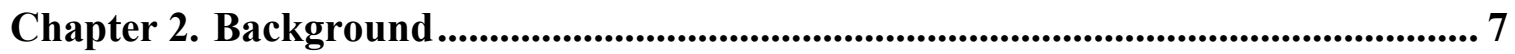

2.1. Source of Power in Digital CMOS Circuit ...................................................... 7

2.1.1 Total Power Reduction Approach ........................................................................... 9

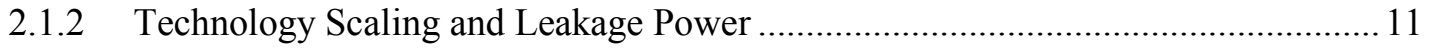

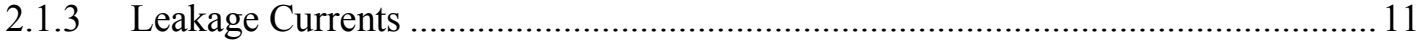

2.2. MOSFET Threshold Voltage ................................................................... 12

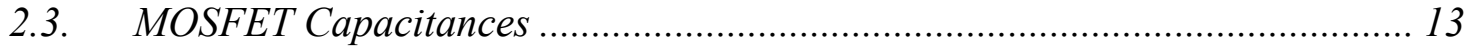

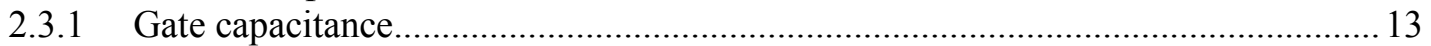

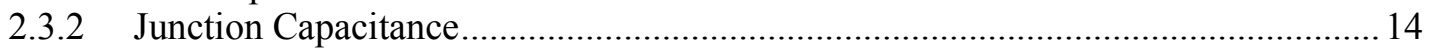

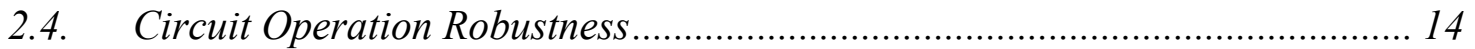

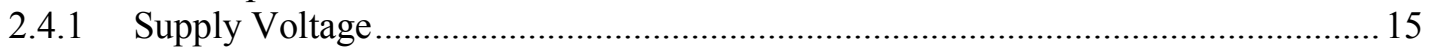

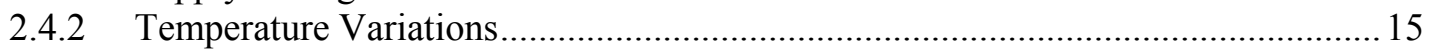

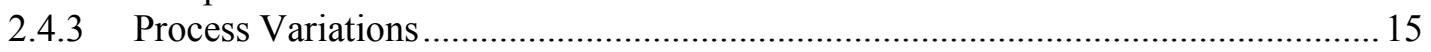

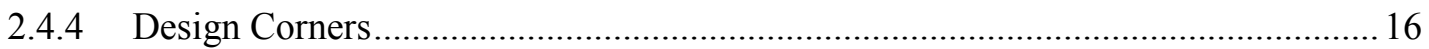

2.5. Effect of Channel Length Variations on Threshold Voltage ............................ 17

2.6. Sequential Computing............................................................................ 17

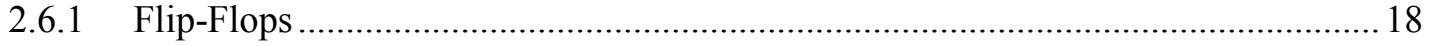

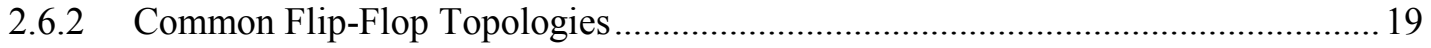

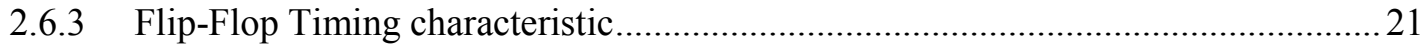

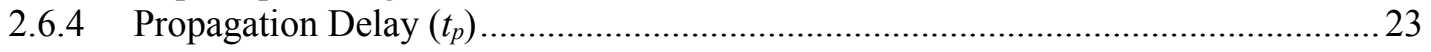




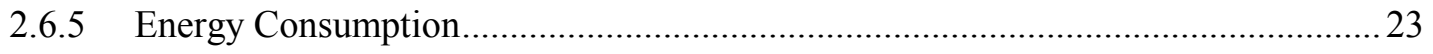

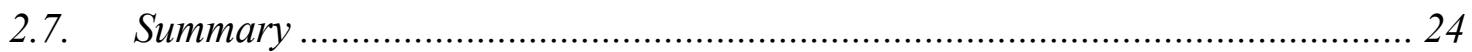

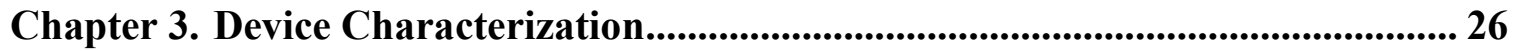

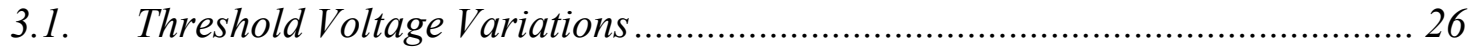

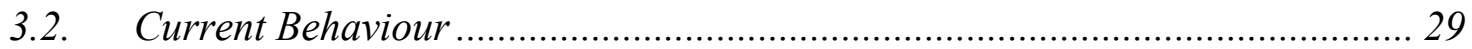

3.3. Comparing Charge and Discharge Currents ........................................... 36

3.4. Improving Robustness of Subthreshold Circuits by Biasing the Body.............. 37

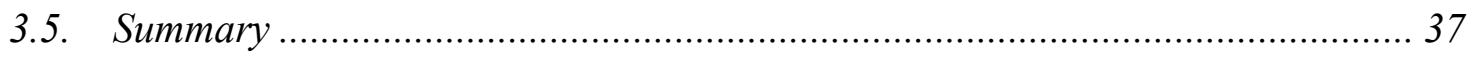

Chapter 4. Proposed Design and Testing Approach ................................................ 38

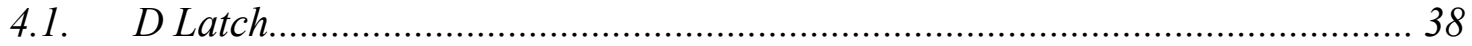

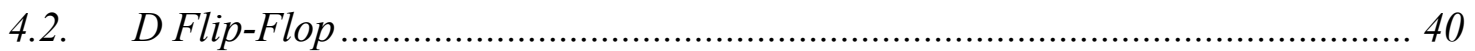

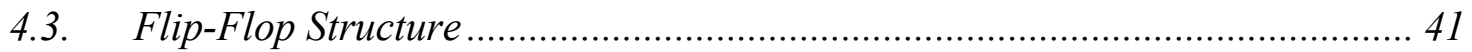

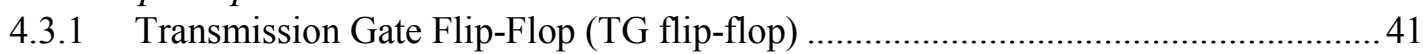

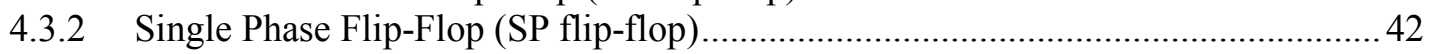

4.4. Multi-Threshold MOSFETs ..................................................................... 43

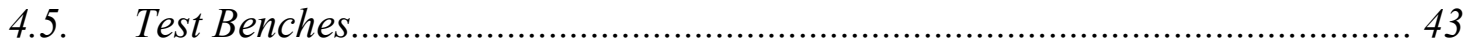

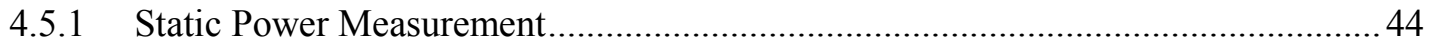

4.5.2 Dynamic Power Consumption Measurement............................................................ 45

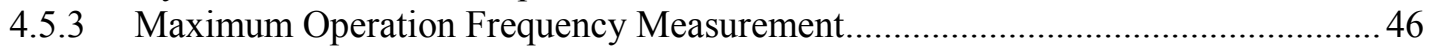

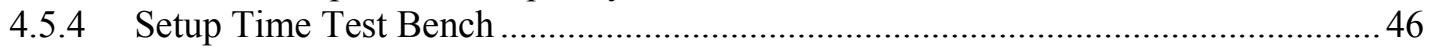

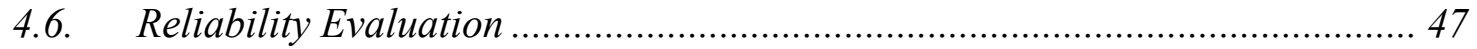

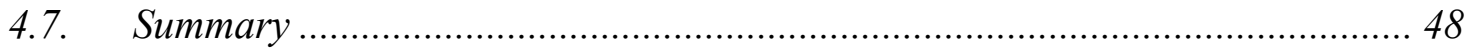

Chapter 5. Reliable Subthreshold Flip-Flop Design Using Multi-Threshold

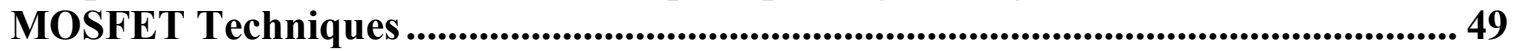

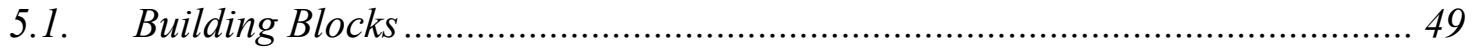

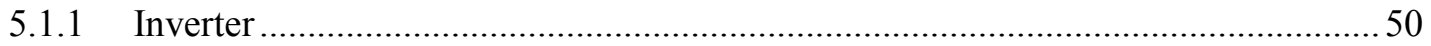

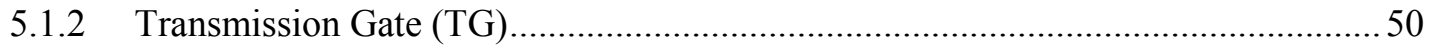

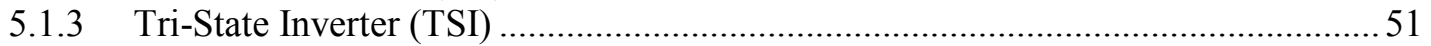

5.1.4 Inverter, Tri-State, and Transmission Gate Inverter Simulation Results ................. 51

5.2. Design of PVT-Tolerant Subthreshold Flip-Flops...................................... 53

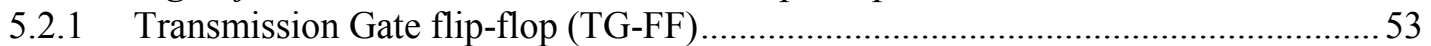

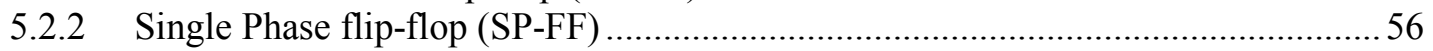

5.3. Comparison between $T G$ and SP flip-flops .............................................. 59

5.4. Effect of Temperature and supply Voltage Variations.................................. 62

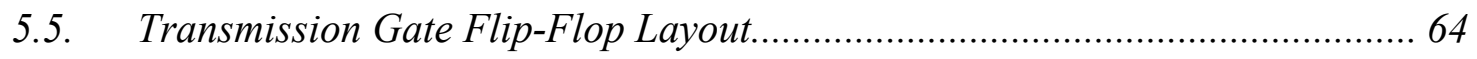

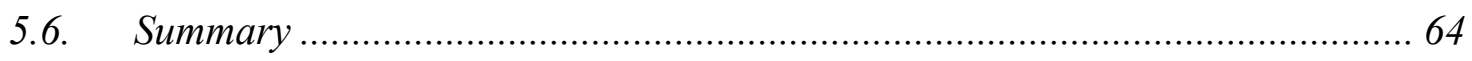


Chapter 6. Improved Device Characterization technique .............................................. 65

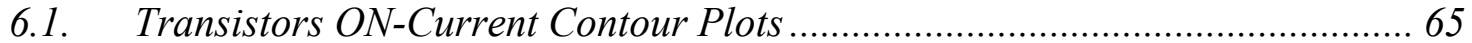

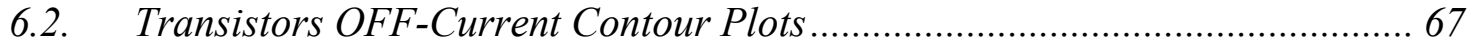

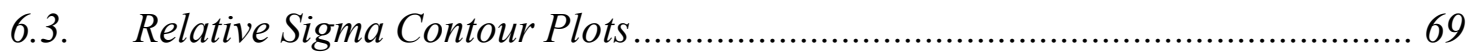

6.4. Low Threshold Voltage Transistors ON and OFF-Current Contour Plots..... 71

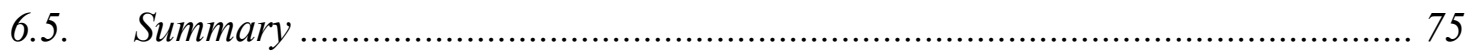

Chapter 7. Reliable Subthreshold Flip-Flop Design by transistor Sizing ................. 76

7.1. Design of PVT-Tolerant Subthreshold Flip-Flops...................................... 76

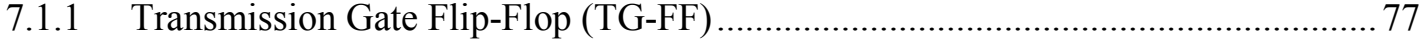

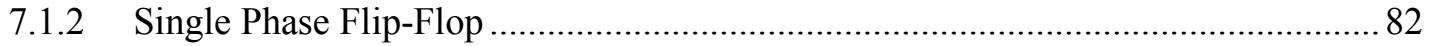

7.2. Comparison between TG flip-flop and SP-flip-flop ................................... 87

7.3. Effect of Temperature and Supply Voltage Variations ................................. 92

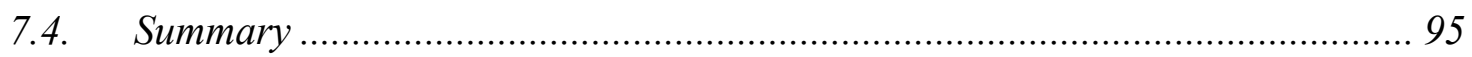

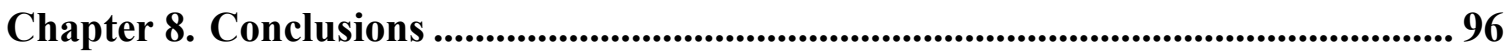

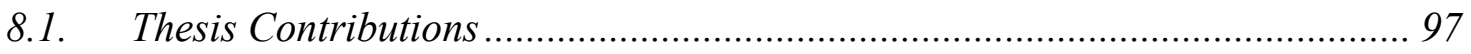

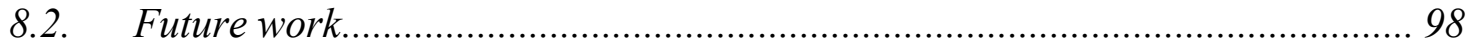

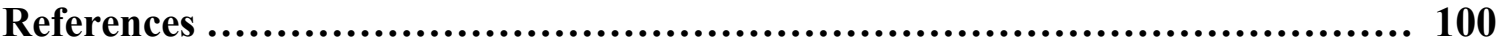

Appendix A: Body-Bias Mechanisms ........................................................................ 104

Appendix B: Layout of Transmission Gate Flip-Flop ........................................... 112 


\section{List of Figures}

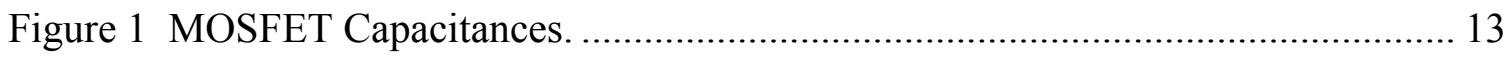

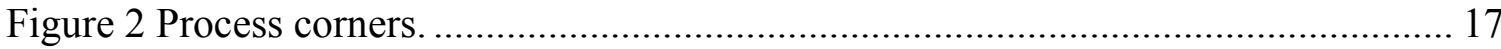

Figure 3 Clocked sequential system. .................................................................. 18

Figure 4 Positive edge-triggered register based on two latches configuration................. 20

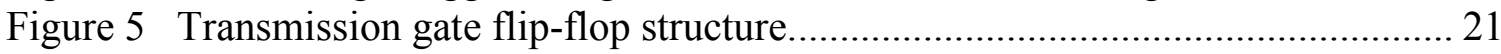

Figure 6 Flip-Flop timing diagram. ....................................................................... 22

Figure $7 \log \left(\mathrm{I}_{\text {on }}\right)$ as a function of $V_{g s}$ for a minimum size hvt-NMOS transistor in the 65 $\mathrm{nm}$ technology. .27

Figure $8 V_{t h}$ versus $L\left(W=W_{\min }=120 \mathrm{~nm}, V_{d d}=200 \mathrm{mV}, 65 \mathrm{~nm}\right.$ process, NMOS). ...... 27

Figure $9 V_{t h}$ versus $L\left(\mathrm{~W}=\mathrm{W}_{\min }=120 \mathrm{~nm}, \mathrm{~V}_{\mathrm{dd}}=200 \mathrm{mV}, 65 \mathrm{~nm}\right.$ process, $\left.\mathrm{PMOS}\right) \ldots \ldots . .28$

Figure $10 V_{t h}$ versus $W\left(L=L_{m i n}=60 \mathrm{~nm}, V_{d d}=200 \mathrm{mV}, 65 \mathrm{~nm}\right.$ process, NMOS $) \ldots \ldots . .28$

Figure $11 V_{t h}$ versus $W\left(L=L_{\min }=60 \mathrm{~nm}, V_{d d}=200 \mathrm{mV}, 65 \mathrm{~nm}\right.$ process, PMOS $)$........ 29

Figure 12 Test Benches for measuring (a) NMOS ON current, (b) NMOS OFF current,

(c) PMOS ON current, and (d) PMOS OFF current. 30

Figure 13 Test benches used for gate currents measurement (a) NMOS gate current (b)

PMOS gate current.

Figure 14 Test benches used for junction currents measurement (a) NMOS junction

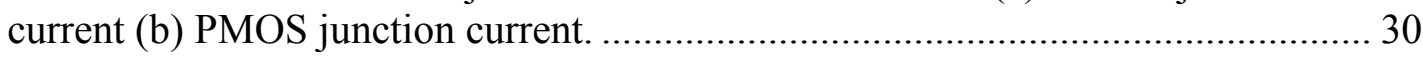

Figure $15 \mathrm{I}_{\mathrm{ON}}$ versus $W\left(L=L_{\min }=60 \mathrm{~nm}, V_{d d}=200 \mathrm{mV}, 65 \mathrm{~nm}\right.$ process $) \ldots \ldots \ldots \ldots \ldots \ldots \ldots . . . . . .32$

Figure $16 I_{\mathrm{oN}}$ versus $L\left(\mathrm{~W}=\mathrm{W}_{\min }=120 \mathrm{~nm}, \mathrm{~V}_{\mathrm{dd}}=200 \mathrm{mV}, 65 \mathrm{~nm}\right.$ process $) \ldots \ldots \ldots \ldots \ldots \ldots . .33$

Figure 17 Ioff versus $W\left(L=L_{\min }=60 \mathrm{~nm}, V_{d d}=200 \mathrm{mV}, 65 \mathrm{~nm}\right.$ process $)$.................. 34

Figure 18 Ioff versus $L\left(\mathrm{~W}=\mathrm{W}_{\min }=120 \mathrm{~nm}, \mathrm{~V}_{\mathrm{dd}}=200 \mathrm{mV}, 65 \mathrm{~nm}\right.$ process $)$.............. 35

Figure 19 Test benches used for charging and discharging of NMOS............................ 36

Figure 20 Logic Diagram for a NAND-based (transparent-high) D latch......................... 39

Figure 21 Logic Diagram for an inverter latch. ............................................................... 39

Figure 22 Diagram for a (transparent-high) D latch based on Transmission Gates (TGs).

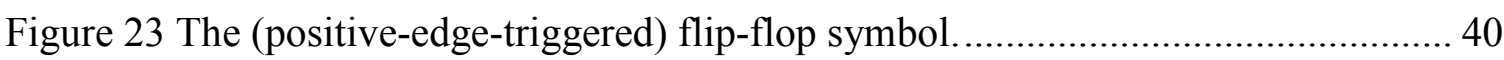

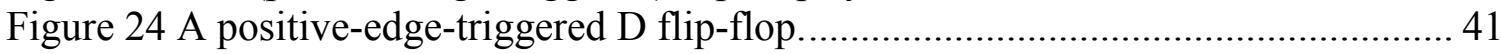

Figure 25 Transmission Gate Flip-Flop circuit......................................................... 41

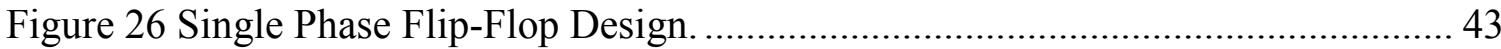

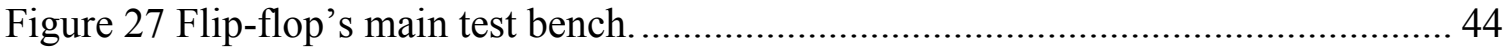

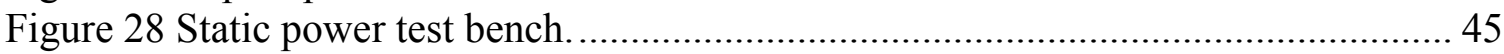

Figure 29 Test bench maximum operation frequency measurement. ................................ 46

Figure 30 The setup-time test bench of TG flip-flop................................................... 47

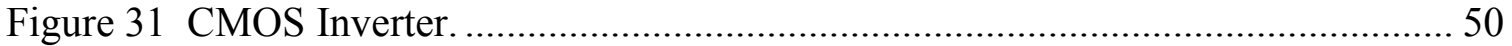

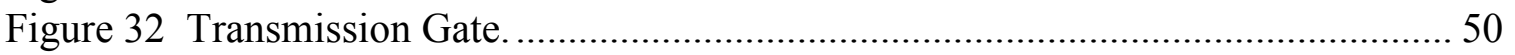

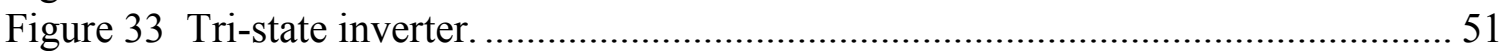

Figure 34 Relative sigma of TSI, and TG $\left(L=L_{\min }=60 \mathrm{~nm}, W=W_{\min }=120 \mathrm{~nm}, V_{d d}=200\right.$

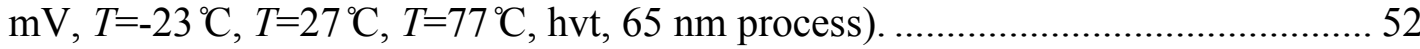

Figure 35 Transmission gate flip-flop schematic.......................................................... 53 
Figure 36 Transient response of minimum size TG flip-flop at (a) SS corner, (b) SF

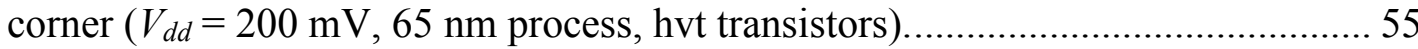

Figure 37 Number of passed of TG flip-flop for different devices ( $V_{d d}=200 \mathrm{mV}, 65 \mathrm{~nm}$

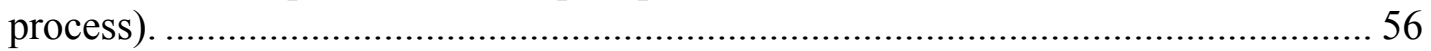

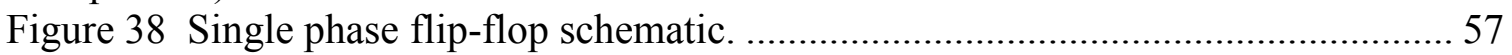

Figure 39. Transient response of minimum size of SP flip-flop at (a) FS corner............. 58

Figure 40 Number of passed of SP flip-flop yield for different devices ........................ 59

Figure 41 Total, static and dynamic power of TG and SP flip-flop versus $V_{d d} \ldots \ldots \ldots \ldots . . . . .59$

Figure 42 Total, static and dynamic power of TG and SP flip-flop versus $T$................. 60

Figure $43 T_{c-q}$ of TG and SP flip-flop versus $V_{d d}(T=100 \mu \mathrm{s}, 65 \mathrm{~nm}$ process, multi-

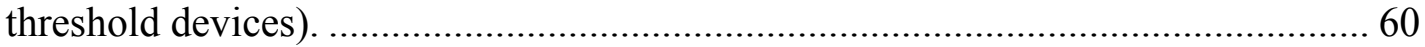

Figure 44 Energy per cycle versus $V_{d d}(65 \mathrm{~nm}$ process, multi-threshold devices).......... 61

Figure $45 I_{O N}$ versus $L$ and $W\left(V_{d d}=200 \mathrm{mV}, 28 \mathrm{~nm}\right.$ process, svt-NMOS $) \ldots \ldots \ldots \ldots \ldots \ldots . . . . . . . .66$

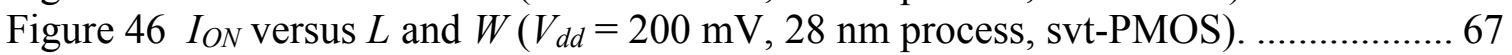

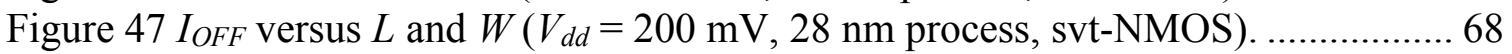

Figure $48 I_{O F F}$ versus $L$ and $W\left(V_{d d}=200 \mathrm{mV}, 28 \mathrm{~nm}\right.$ process, svt-PMOS $) \ldots \ldots \ldots \ldots \ldots \ldots . . . . . . .69$

Figure 49 Relative sigma of NMOS ON-current $\left(V_{d d}=200 \mathrm{mV}, 28 \mathrm{~nm}\right.$ process $)$.......... 70

Figure 50 Relative sigma of PMOS ON-current ( $V_{d d}=200 \mathrm{mV}, 28 \mathrm{~nm}$ process)......... 71

Figure $51 I_{O N}$ versus $L$ and $W\left(V_{d d}=200 \mathrm{mV}, 28 \mathrm{~nm}\right.$ process, lvt-NMOS).................. 72

Figure $52 I_{O N}$ versus $L$ and $W\left(V_{d d}=200 \mathrm{mV}, 28 \mathrm{~nm}\right.$ process, lvt-PMOS $) \ldots \ldots \ldots \ldots \ldots \ldots . . . . . . . . .73$

Figure $53 I_{O F F}$ versus $L$ and $W\left(V_{d d}=200 \mathrm{mV}, 28 \mathrm{~nm}\right.$ process, lvt-NMOS $) \ldots \ldots \ldots \ldots \ldots \ldots . . . . . . . . .74$

Figure $54 I_{O F F}$ versus $L$ and $W\left(V_{d d}=200 \mathrm{mV}, 28 \mathrm{~nm}\right.$ process, lvt-PMOS $) . \ldots \ldots \ldots \ldots \ldots . . . . . .75$

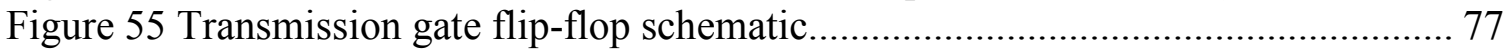

Figure 56 Transient response of minimum size TG flip-flop at FS and SF corner

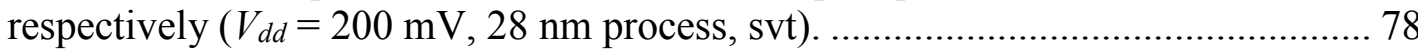

Figure 57 Number of passed of TG flip-flop yield for different sizes ( $V_{d d}=200 \mathrm{mV}, 28$ $\mathrm{nm}$ process, $\mathrm{svt}$ ).

Figure 58 The rise and fall time of TG flip-flop output $\left(V_{d d}=200 \mathrm{mV}, 28 \mathrm{~nm}\right.$ process,

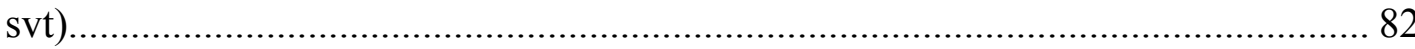

Figure 59 Single phase flip-flop schematic. ......................................................... 83

Figure 60 Transient response of TG flip-flop at SF and FS corner respectively $\left(V_{d d}=200\right.$

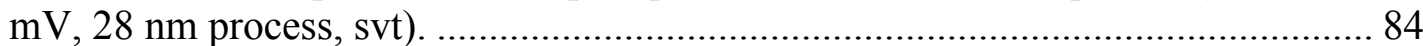

Figure 61 Passed of SP flip-flop yield ( $V_{d d}=200 \mathrm{mV}, 28 \mathrm{~nm}$ process, svt). ................. 85

Figure 62 The output waveform $P$ of first latch of SP flip-flop (a) LN=LN2=45 nm (b)

$\mathrm{WP}=300 \mathrm{~nm}(\mathrm{c}) \mathrm{P}$ of lvt-type PMOS ( $V_{d d}=200 \mathrm{mV}, 28 \mathrm{~nm}$ process, svt)............ 86

Figure 63 Total, static and dynamic power of TG, SP $(\mathrm{WP}=300 \mathrm{~nm})$, and SP (lvt-P) flip-

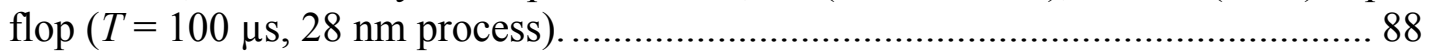

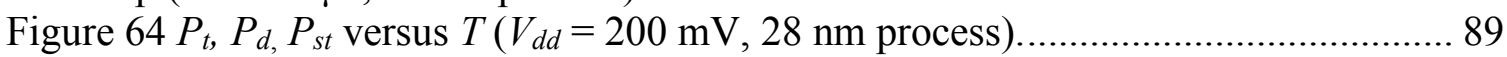

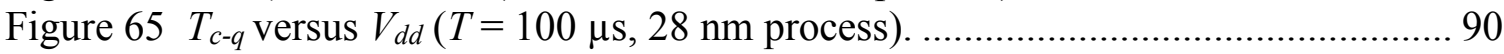

Figure 66 Energy per cycle versus supply voltage in $28 \mathrm{~nm}$ process. .......................... 91 


\section{List of Tables}

Table 1 Summary of some subthreshold circuit applications.

Table 2 NMOS and PMOS transistors leakage currents $\left(L=L_{\min }=60 \mathrm{~nm}, W=W_{\min }=\right.$

$120 \mathrm{~nm}, V_{d d}=200 \mathrm{mV}, 65 \mathrm{~nm}$ process $)$.

Table $3 I_{O N}, I_{O F F}$, and $V_{t h}$ of devices $\left(\left(L=L_{\min }=60 \mathrm{~nm}, W=W_{\min }=120 \mathrm{~nm}, V_{d d}=200\right.\right.$

$\mathrm{mV}, T=27^{\circ} \mathrm{C}, 65 \mathrm{~nm}$ process).

Table 4 Process variation effect on $I_{O N}\left(L=L_{\min }=60 \mathrm{~nm}, W=W_{\min }=120 \mathrm{~nm}, V_{d d}=200\right.$

$\mathrm{mV}, T=27^{\circ} \mathrm{C}, 65 \mathrm{~nm}$ process).

Table 5 Average currents hvt- NMOS and hvt-PMOS $\left(L=L_{\min }=60 \mathrm{~nm}, W=W_{\min }=\right.$

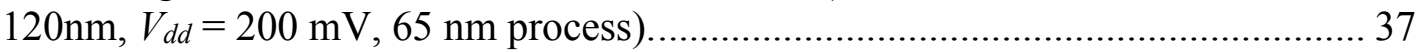

Table $6 T_{c-q}$ and $P_{t}$ of inverter, TSI, and TG $\left(L=L_{\min }=60 \mathrm{~nm}, W=W_{\min }=120 \mathrm{~nm}, V_{d d}=\right.$

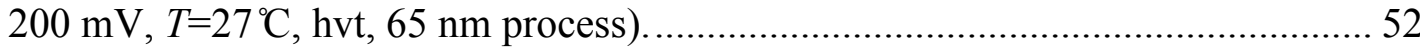

Table 7 Mean and sigma of TSI and TG $\left(L=L_{\min }=60 \mathrm{~nm}, W=W_{\min }=120 \mathrm{~nm}, \ldots \ldots \ldots \ldots . . .52\right.$

Table 8 The simulation results of flip-flops $\left(V_{d d}=200 \mathrm{mV}, T=100 \mu \mathrm{s}, 65 \mathrm{~nm}\right.$ process, multi-threshold devices).

Table 9. The simulation results of flip-flops $\left(V_{d d}=170 \mathrm{mV}, T=100 \mu \mathrm{s}, 65 \mathrm{~nm}\right.$ process,

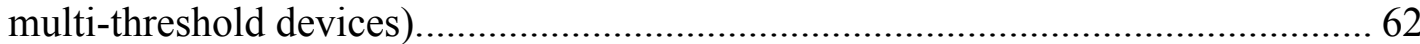

Table 10. Mean, Sigma, minimum, and maximum delay of TG and SP flip-flop $(T=100$ us, $65 \mathrm{~nm}$ process, multi-threshold devices).

Table 11. The power, energy, and frequency of inverter chain $\left(V_{d d}=200 \mathrm{mV}, T=100 \mathrm{us}\right.$, $28 \mathrm{~nm}$ process, svt, TT).

Table 12 The rising and falling of SP flip-flop output ( $V_{d d}=200 \mathrm{mV}, 28 \mathrm{~nm}$ process, svt).

Table 13 Simulation results of flip-flops ( $V_{d d}=200 \mathrm{mV}, T=100 \mu \mathrm{s}, 28 \mathrm{~nm}$ process $) \ldots .92$

Table 14 The simulation results of flip-flop ( $V_{d d}=150 \mathrm{mV}, T=100 \mu \mathrm{s}, 28 \mathrm{~nm}$ process).

Table 15 Mean, Sigma, minimum, and maximum delay of TG and SP flip-flop ( $T=100$ $\mu \mathrm{s}, 28 \mathrm{~nm}$ process). 


\section{List of Acronyms}

$\begin{array}{ll}\text { Acronym } & \text { Definition } \\ \text { ACS } & \text { Add-Compare-Select } \\ \text { CLK } & \text { Clock } \\ \text { CMOS } & \text { Complementary metal-oxide-semiconductor } \\ \text { CPU } & \text { Central Processing Unit } \\ \text { D } & \text { Data } \\ \text { DIBL } & \text { Drain Induced Barrier Lowering } \\ \text { DRO } & \text { Dynamic Ring Oscillator } \\ \text { DTMOS } & \text { Dynamic Threshold Voltage } \\ \text { EDP } & \text { Energy Delay Product } \\ \text { FD-SOI } & \text { Fully Depleted Silicon On Insulator } \\ \text { FF } & \text { Flip-Flop } \\ \text { FFBB } & \text { Flip-Flop with Body Biasing } \\ \text { FFT } & \text { Fast Fourier Transform } \\ \text { FinFET } & \text { Fin shaped Field Effect Transistor } \\ \text { GDI } & \text { Gate Diffusion Input } \\ \text { GIDL } & \text { Gate Induced Drain Leakage } \\ \text { HVT } & \text { High-Voltage-Threshold } \\ \text { INV } & \text { Inverter } \\ \text { INWE } & \text { Inverse Narrow Width Effect } \\ \text { LP } & \text { Low Power } \\ \text { LVT } & \text { Low-Voltage-Threshold } \\ \text { MOSFET } & \text { Metal-Oxide-Semiconductor Field Effect Transistor } \\ \text { MUX } & \text { Multiplexer } \\ \text { NAND } & \text { Logic gate that implements negated AND } \\ \text { NMOS } & \text { n-Channel MOSFET } \\ \text { PBB } & \text { Path Delay with Body Biasing } \\ \text { PDAs } & \text { Personal Digital Assistant } \\ \text { PDP } & \text { Power Delay product } \\ \text { PMOS } & \text { p-Channel MOSFET } \\ \text { PTL } & \text { Pass Transistor Logic } \\ \text { RO } & \text { Ring Oscillator } \\ \text { SP } & \text { Single Phase } \\ \text { SRAM } & \text { Static Random-Access Memory } \\ \text { SR } & \text { Shift Register } \\ \text { SVT } & \text { Standard-Voltage-Threshold } \\ \text { TG } & \text { Transmission Gate } \\ \text { TSI } & \text { Tri-State Inverter } \\ & \\ & \end{array}$




\section{Chapter 1. Introduction}

One of the important design specifications for electronic circuits is power consumptions. As the demand for ultra-low power (ULP) applications, such as sensor networks, medical devices and static random access memory (SRAM) cells continues to grow, subthreshold CMOS circuits have become a subject of interest for researchers [1] [2]. The minimum energy consumption is achieved when the supply voltage is scaled below the threshold of MOSFET, that is, subthreshold operation. Hence, subthreshold circuits are introduced based on the most fundamental method to decrease energy dissipation, which is voltagescaling.

Subthreshold circuits take advantage of the main leakage current in commonly used superthreshold counterparts, known as the subthreshold current, as their driving force. This current is governed by a different equation compared to the saturation and triode currents. Consequently, new challenges are introduced for digital / analog circuits designed to operate in the subthreshold region. Some of these challenges include lower speed and higher sensitivity to process, voltage and temperature (PVT) variations compared to superthreshold circuits. Increasing the reliability at minimal performance and energy cost is essential for subthreshold circuits.

This thesis addresses the design of efficient and reliable flip-flops for subthrehold operation. It compares the reliability, power consumption, and speed of two major CMOS flip-flop topologies to identify the most suitable one. It also recommends specific multithreshold and transistor sizing technique to improve the reliability of flip-flops for subthreshold operation. The circuits design kits used to prove the concept are TSMC $65 \mathrm{~nm}$ LP (low power) CMOS and STM 28nm FD (fully depleted) SOI CMOS technologies.

\subsection{Motivation}

Flip-flops play an important role in power dissipation and performance of sequential digital systems, as they are the building block of such circuits. They are used in abundance with 
many applications, such as, event detection, data synchronization, frequency division, shift registers, counters and finite state machines [3]. Hence, to improve the performance of synchronized sequential logics, it is essential to focus on enhancing the design of flip-flops with regards to reliability, power consumption and delay.

Subthreshold circuits involve design challenges, such as, increased delay and sensitivity to PVT variations. Consequently, addressing these issues for CMOS flip-flops operating in subthreshold region is crucial for robust design of ULP sequential digital circuits. Due to the timing constraint, designing energy and speed efficient as well as reliable flipflops becomes a challenge.

Researchers have proposed different topologies to improve the reliability of CMOS flip-flops operating in subthreshold region [3]. However, a detailed study and comparison of different flip-flop topologies operating in subthreshold region using appropriate device choice or sizing has not been completed. This investigation, which involves increasing reliability while maintaining low energy dissipation and delay, remains a promising research topic.

\subsection{Thesis Objectives}

This thesis addresses the challenge of designing reliable flip-flops for subthreshold operation. The main objective is to introduce circuit techniques to improve the reliability of subthreshold flip-flops without sacrificing power and speed, leading towards identification of the most suitable subthreshold flip-flop topology.

To achieve this objective, the $65 \mathrm{~nm}$ and $28 \mathrm{~nm}$ CMOS kits developed by TSMC are used and the following milestones are set.

1. Investigating the effect of changing the channel length and width of a MOSFET on its threshold voltage, ON-current, and OFF-current.

2. Performing a comparative analysis of major flip-flop topologies for subthreshold operation in terms of reliability, delay, and power consumption.

3. Identifying the preferable transistor sizing technique in terms of tolerating PVT variations.

4. Proposing and investigating the use of multi-threshold and transistor sizing techniques to improve the robustness of subthreshold flip-flops. 
5. Performing Monte Carlo simulation to assess the reliability of the proposed flip-flop designs.

6. Finally, recommending the most suitable flip-flop topology for subthreshold operation.

\subsection{Previous Work}

As supply voltages continue to scale with new generations of CMOS technologies, subthreshold operation is a predictable conclusion in the semiconductor roadmap. Thus, research activity in subthreshold logic has gradually increased since the early nineties. One of the first papers to describe digital logic at such low voltages investigated the basic CMOS inverter operating in subthreshold [1]. This configuration was analytically evaluated and energy delay product (EDP) was identified as the appropriate metric to compare two designs [1]. Some papers have looked into different subthreshold circuit implementations for minimizing power consumption and optimizing device performance [2] [4].

The effect of voltage scaling for reducing the power consumption of a CMOS counter was explored by Vittoz [5]. For a long time, transistor operation in the subthreshold region has been a popular method for reducing the power consumption. In 1972, Swanson reported the lower bounds of supply voltage about $200 \mathrm{mV}$ at room temperature [6].

Calhoun shows that that subthreshold operation can be considered as the most energy efficient solution for ULP applications [3]. In this paper, subthreshold logic and memory design methods were developed and the results were verified using a fast Fourier transform (FFT) processor [7].

Some subthreshold circuit applications are listed in Table 1. Research developments focusing on minimizing energy consumption [4], [8], [7] and optimizing devices performance [5] are summarized in this table. 
Table 1 Summary of some subthreshold circuit applications.

\begin{tabular}{|r|l|r|}
\hline Year & \multicolumn{1}{|c|}{ Application } & Reference \\
\hline 2005 & FFT-Processor at $180 \mathrm{mV}$ & {$[7]$} \\
\hline 2006 & SRAM circuit at $190 \mathrm{mV}$ & {$[9]$} \\
\hline 2007 & Programmable Register file at $200 \mathrm{mV}$ & {$[10]$} \\
\hline 2008 & CPU processor below $200 \mathrm{mV}$ & {$[11]$} \\
\hline 2010 & Carbon- Nanotube for micro sensor at $200 \mathrm{mV}$ & {$[12]$} \\
\hline
\end{tabular}

Some papers have presented ways reduce PVT variations. Soeleman shows superior robustness to temperature and process variations using dynamic threshold MOS (DTMOS) logic [13]. However, due to body biasing of devices, circuits should be implemented in triple well technologies. A novel circuit implementation in subthreshold region using source coupled logic (SCL) in order to increased reliability is presented by Tajali [14]. A novel method for increasing subthreshold frequency of operation by using parallel transistor stacks is presented by Muker and Shams [15]. In this paper, a new shifter design using multi-threshold CMOS (MT-CMOS) techniques in $90 \mathrm{~nm}$ process was recommended [16].

A comparative study on different topologies of CMOS flip-flops operating in the subthreshold region was completed [17]. This paper shows that hybrid latch flip-flop (HLFF) achieves the smallest increase in clock to output delay compared to other structures due to a much smaller increase in setup time with decreased supply voltage. For energy efficient operation at ultra-low voltages, HLFF achieves the smallest EDP during switching. However, reliability of flip-flops was not investigated for these different structures.

Several recent publications have proposed and tested flip-flop designs for operation in the subthreshold region [18]. A transmission gate flip-flop is modified by cutting off the feedback line to eliminate ratio sizing in paper that has been reported by Wang [3] . As a result, non-ratioed circuit styles provide more robust functionality in subthreshold.

Transmission gate flip-flop structure is modified by Rabaey [19]. In this book, a reduced load clock load static flip-flop is proposed, where the feedback transmission gate can be eliminated by directly cross-coupling inverters. The penalty for this design includes 
the reverse conduction in the second stage that can affect the state of the first latch. The solution is introduced by using a weaker inverter in the second latch [3].

A number of flip-flop configurations have been tested and modified by Alstad [18]. In this thesis work, different flip-flop designs, commonly used in superthreshold circuits, are compared to ones operating in subthreshold. According to process corner simulations, a PowerPC 603 type flip-flop operates successfully in all corners in a $65 \mathrm{~nm}$ process down to a power supply voltage of $125 \mathrm{mV}$. Chavan has proposed a number of other topologies [20]. These designs have been compared and optimized for power reduction at the expense of area and complexity.

Weste shows that true single phase clock dynamic latches and flip-flops can replace the inverter transmission gate with a pair of stages requiring a single clock [21]. These dynamic latches were used on ground-breaking Alpha 21064 microprocessor. In any case, the clock must be reasonably sharp to prevent races when both transistors are partially on. Rabaey investigates single phase static flip-flop structures with regards to clock skew, which is a major problem challenge for flip-flop design. Clock skewing, which leads to clock-overlap [19]. However, single phased flip-flops have not been investigated for subthreshold operation. Hence, the power consumption and reliability of these structures are studied and compared to other flip-flop topologies in this thesis work.

Morgenshtein has presented gate diffusion input (GDI) technique for ULP CMOS design [22]. This paper also presents a detailed comparison between gate diffusion input and pass-transistor logic (PTL) flip-flop configurations. The D flip-flop implementation using gate diffusion technique has been presented and developed based on transistor sizing [23]. Two subthreshold flip-flop structures using GDI have been presented and characterized for small area and minimum energy dissipation [23]. However, sensitivities to PVT variations as well as delay have not been examined. Swami has suggested the optimal performance of GDI technique using D flip-flops with regards to power consumption [24]. However, this flip-flop requires tight timing restrictions that do not tolerate process variations.

There is no research reported on the comparison between major flip-flop configurations for subthreshold operation. Furthermore, transistor sizing technique and multithreshold devices have not been incorporated to improve the reliability of flip-flops in the 
literature. In addition, no flip-flop reliability evaluations based on PVT variations and Monte Carlo simulations are addressed in the literature. Decreasing PVT variations for the transmission-gate (TG) and single-phase (SP) flip-flops by using proper device sizes and choosing devices with appropriate threshold voltages is the basis of this work. This achievement has to be fulfilled at minimal energy and delay cost.

\subsection{Thesis Outline}

After this introductory Chapter, the remainder of this thesis is organized as follows: In Chapter 2, the theoretical background of MOSFETs operating in subthreshold region as well as flip-flop topologies is studied. Chapter 3 presents a detailed study on three different types of important transistor parameters by using the $65 \mathrm{~nm}$ CMOS process. In Chapter 4 , the building blocks of flip-flops are presented and compared with regards to power consumption, delay and reliability using the $65 \mathrm{~nm}$ CMOS technology. Chapter 5 explains the details of a reliable subthreshold flip-flop design using multi-threshold technique using the same process.

In Chapter 6, a novel technique based on contour plots for appropriate device sizing is proposed to improve the characteristics of transistors operating in subthreshold using the $28 \mathrm{~nm}$ process. Chapter 7 suggests a transistor sizing technique for reliability improvement of subthreshold flip-flops using the $28 \mathrm{~nm}$ process. Chapter 8 highlights the final remarks and future work based on this thesis. In appendix A, the body biasing methods are presented and compared. In appendix B, the layout of transmission gate flip-flop is implemented and compared to schematics results. 


\section{Chapter 2. Background}

To understand the operation of subthreshold circuits and address their challenges, MOSFET behavior in the subthreshold region needs to be studied. Furthermore, understanding the basics of sequential computations and figures of merits for synchronized circuits is essential for designing flip-flops. In this regard, this chapter is divided into two main sections. The first part presents a theoretical study of MOSFET subthreshold operation characteristics and figures of merit, such as threshold, current, power consumption and reliability. The second section explains the theory behind sequential computing and flipflops.

\subsection{Source of Power in Digital CMOS Circuit}

Low power circuit operation is becoming a gradually important metric for future integrated circuits. Portable battery powered devices, such as, cell phones, pagers, personal digital assistant (PDAs), and portable computers become more complex and prevalent [3]. Hence, the demand for increased battery life requires designers to seek out new technologies and circuit techniques to maintain high performance and long operational lifetimes. In nonportable applications, reducing power dissipation is also becoming an increasingly important issue. In the past, high end microprocessors were engineered, with performance being the primary goal. However, in modern systems, power dissipation can become so large that heat removal becomes a problem.

In modern digital CMOS integrated circuits (IC), power consumption can be attributed to three different components: short circuits, static or leakage power and dynamic or switching power. Short circuit currents occur in CMOS circuits during switching transients, when both NMOS and PMOS devices are "ON". This leakage is usually small in new designed circuits [25]. Dynamic switching power is the dominant component of power consumption and results from the charging and discharging of gate capacitances during signal switching, as shown in Equation (2-1): 


$$
P_{d}=\alpha C_{L} V_{d d}^{2} f_{c l k}
$$

where $P_{d}$ is dynamic power, $\alpha$ is switching activity factor, $C_{L}$ is the total switched capacitance, $V_{d d}$ is the supply voltage, and $f_{c l k}$ is the switching frequency.

The third component of power consumption is static power. Although limited compared to dynamic switching power, static power is becoming more important as scaling trends continue and efforts to achieve ULP circuit operation are become strengthened. The static or leakage power $\left(P_{s}\right)$ in a circuit is given by:

$$
P_{s}=V_{d d} I_{\text {leak }}
$$

where $I_{\text {leak }}$ is the leakage current.

In the models of both by Shockley and Sakurai, the current for $V_{g s}<V_{t h}$ is considered to be " 0 " [21]. However, in reality, even in this region there is a current flowing from the drain to the source. For circuits operating in superthreshold, the subthreshold current is considered a leakage. But for subthreshold circuits this small current is the operation one. This current is given by [21]:

$$
I_{d s}=I_{0} e^{\frac{V_{g s}-V_{t h}}{n V_{T}}}\left(1-e^{-\frac{V_{d s}}{V_{T}}}\right)
$$

where $I_{0}=\mu_{0} C_{o x} W / L V_{T}^{2} e^{1.8}, \mu_{0}$ is the charge carriers' motilities of MOSFET, $C_{o x}$ is gate oxide capacitance, $W$ is transistor gate width, $L$ is transistor gate length, and $V_{T}=K T / q$ is the thermal voltage, that the value of which is $26 \mathrm{mV}$ at $300 \mathrm{~K}, n$ is the subthreshold slope factor that varies by depletion region and is in the range of 1.3 to $1.7, V_{g s}$ is gate source voltage, $V_{d s}$ is drain source voltage, and $V_{t h}$ is threshold voltage. $n$ is expressed as [26]

$$
n=1+\frac{C_{d e p}}{C_{o x}}
$$

equation (2-3) exposes two properties. First, as $V_{d s}$ exceeds a few $V_{T}, 1-e^{\frac{V_{d s}}{V_{T}}}$ becomes "1" and the current becomes independent of $V_{d s}$. Second, the slope of $I_{d s}$ on a semi-logarithmic scale equals 


$$
\frac{\partial\left(\log _{10} I d s\right)}{\partial V_{g s}}=\log _{10} e \frac{1}{n V_{T}}
$$

The inverse of this quantity is called the sub-threshold slope $(S)$

$$
S=n V_{T} \ln 10
$$

In order to turn off the transistor by lowering $V_{g s}$ in the sub-threshold region, $S$ must be as small as possible. Parameter $S$ is normally in the range of 70 to $100 \mathrm{mV} / \mathrm{dec}$ [27].

\subsubsection{Total Power Reduction Approach}

The power consumption of a system sets up how much energy is consumed per operation, and how much heat is dissipated. The upper power limits determine the maximum number of transistors that are integrated on a single chip, a heat removal system, a chip package, and especially, the frequency at which the transistor switches [19]. As previously explained, the power consumption is composed of two components: dynamic power and static power. Dynamic power can also be expressed as

$$
P_{d}=P_{s w}+P_{s c}
$$

where $P_{S w}$ is switching power due to the switching activity, and $P_{s c}$ is short circuit power due to short circuit current. The short circuit current occurs as pull-up and pull-down networks are partially on while the input switches. In nanometer processes, short circuit current has become almost negligible.

Static power has two parts, as

$$
P_{s}=P_{\text {leak }}+P_{\text {cont }}
$$

where $P_{\text {leak }}$ is leakage power due to leakage current, and $P_{\text {cont }}$ is contention power due to contention current. The contention currents are produced due to contention between NMOS (pull-down) and PMOS (pull-up) devices when the output is "0". Static CMOS circuits 
have no contention current. These two sources of power consumption are represented by the total power $\left(P_{t}\right)$ as [21]

$$
P_{t}=P_{d}+P_{s}=\alpha C_{L} V_{d d}^{2} f_{c l k}+V_{d d} I_{\text {leak }}
$$

where $\alpha$ is activity factor, $C_{L}$ is output capacitance, $V_{d d}$ is supply voltage, $f_{c l k}$ is clock frequency, and $I_{\text {leak }}$ is leakage current.

Subthreshold leakage currents will become an important component of total power dissipation in future technologies. Scaling theory alone dictates that subthreshold leakage currents will continue to become more important in overall power dissipation. Likewise, for low power scaling, the optimum energy point for $V_{d d}$ and $V_{t h}$ will also approve to a larger subthreshold leakage component. Although total power dissipation during the active mode can be reduced by scaling, further power gains can be achieved if subthreshold leakage currents are controlled. In order to reduce the dynamic power component, the first goal is aggressive supply voltage scaling. Reducing $V_{d d}$ leads to quadratic power reductions, while decreasing switching activity, operating frequency and load capacitance results in linear decreases of dynamic power. For the static power component, besides the supply voltage scaling, which provides linear power reductions, the objective is to keep the leakage current as low as possible. Leakage power increases exponentially for each technology node, and eventually becomes the dominant component of $P_{t}$ as the technology scales beyond $65 \mathrm{~nm}$ [28], [29]. This is why low power strategies, especially those for static power reduction, are necessary at almost any design level in recent digital circuits.

The power dissipation of high performance applications, such as, microprocessors, digital signal processors, and RAMs has increased with progress in CMOS technologies, where the design emphasis has been on maximizing speed. The increased power consumption raises a chip temperature which leads to electro migration reliability problems, and degradation in the performance of the device. Thus, lowering the power dissipation is crucial for high performance very large scaled integrated (VLSI) designs [30]. Also, applications are emerging for which energy consumption is the key metric, and the speed of operation has become less relevant. Generally, energy-constrained VLSI applications such as micro-sensor networks and nodes, have low activity rates and speed requirements, but the 
main concern is to lengthen battery life. Ideally, the power consumption of these systems should decrease to the extent that they can harvest energy from environmental resources, such as solar power, thermal gradient, radio-frequency (RF) and mechanical vibration [31]. Such ultra-low-power applications have established a significant role for subthreshold circuits [3].

\subsubsection{Technology Scaling and Leakage Power}

Over the last three decades, the evolution of CMOS technology has resulted in substantial device scaling to achieve density, speed, and power improvements, as predicted by Moore's Law [32]. The direct result of device scaling is reduced intrinsic capacitance, enabling a faster switch. Simultaneously, power supply voltage scaling has reduced the switching energy. To maintain the speed enhancement for each technology node, the threshold voltage must also be scaled down in order to retain enough gate overdrive $V_{d d} / V_{t h}$. However, reducing the $V_{t h}$ can result in an exponential increase in the subthreshold leakage current, as shown in Equations (2-3) in the next section. The oxide thickness scaling, which is required to maintain reasonable short channel effects, results in a considerable amount

of direct oxide tunneling of leakage current. Finally, the higher substrate doping density and the application of the halo profiles to reduce short channel affects in scaled devices causes substantially large junction band-to-band tunneling leakage [21]. In this way, among the seven leakage mechanisms in scaled devices, there are three major sources of leakage currents in CMOS transistors that are named: subthreshold, oxide tunneling, and reverse bias p-n junction [33]. The magnitudes of each component depend strongly on the device constitution; that is, oxide thickness, channel length, and doping [34].

\subsubsection{Leakage Currents}

There are four major sources of leakage currents in CMOS transistors.

\section{- Subthreshold Leakage $\left(I_{\text {sub }}\right)$}

This current flows between the drain and the source of a MOSFET when the gatesource voltage is below the threshold voltage. The magnitude of this leakage current is a function of the temperature, supply voltage, device size, and process parameters [27]. 


\section{- Gate Induced Drain Leakage $\left(I_{G I D L}\right)$}

This current is due to a high electric field in the drain junction of a MOSFET. Thinner oxide thickness and higher supply voltage increases the potential between the gate and drain, which results in higher GIDL leakage [21].

\section{- Tunneling into and through Gate-Oxide $\left(I_{G}\right)$}

The oxide thickness has become smaller, and the channel length is reduced in modern technologies. The reduction of the oxide thickness results in the tunneling of electrons from the gate to the substrate and from the substrate to the gate, which is called the gate-oxide tunneling current [21].

\section{- PN Junction Reverse-Bias current $\left(I_{j u n c}\right)$}

This current is produced by the drain- to-body and source-to-body junctions, which are typically reverse biased [21].

By using explained leakage current, static power from Equation (2-2) is expressed as:

$$
P_{s}=V_{d d} I_{\text {leak }}=\left(I_{\text {sub }}+I_{G}+I_{\text {junc }}\right)
$$

\subsection{MOSFET Threshold Voltage}

To have a better understanding of the effects of transistor sizing on threshold voltage and, consequently, on current, a simple quantitative expression is introduced for the threshold voltage [18]

$$
V_{t h}=V_{f b}+\emptyset_{s t}+\frac{Q_{d e p}}{C_{o x}}
$$

where $V_{f b}$ is the flat-band voltage, $\varnothing_{s t}$ is surface potential at the threshold edge, $Q_{d e p}$ is the depletion region charge, and $C_{o x}$ is gate oxide capacitance. The first and second terms in Equation (2-11) are fixed for a given technology and depend on the doping levels of the substrate and poly silicon [35]. But the third term is dependent on the transistor sizes. This means that changing the size of a transistor changes its threshold voltage. The effect of 
channel width and length are two important phenomena that relate the threshold voltage variations to the transistor dimensions.

\subsection{MOSFET Capacitances}

In order to understand the dynamic behavior of MOSFETs, as well as the current and the threshold voltage, we need to study the different capacitances of MOSFET. As shown in Figure 1, the main capacitances of a MOSFET are the gate $\left(C_{G}\right)$ and the junction $\left(C_{J}\right)$ capacitances [21].

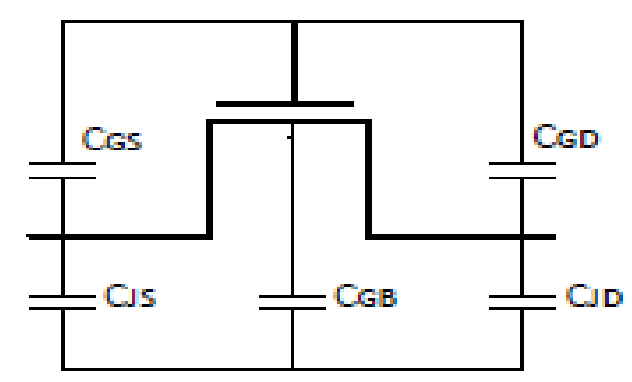

Figure 1 MOSFET Capacitances.

\subsubsection{Gate capacitance}

The gate capacitance consists of three components [19]: gate-drain $\left(C_{G D}\right)$, gate-source $\left(C_{G S}\right)$, and gate-bulk $\left(C_{G B}\right)$ capacitances. The gate-bulk capacitance is estimated by

$$
C_{G B}=C_{o x} W L
$$

where $W$ is width of transistor gate, $L$ is length of transistor gate, and $C_{o x}$ is oxide gate capacitance. The other components of the gate capacitance, gate-drain and gate-source capacitances are caused by the source and drain extensions of the MOSFET under the gate oxide. They are expressed as [19]:

$$
C_{G S}=C_{G D}=C_{o x} x_{d} W
$$


where $x_{d}$ is the extension of the source or drain under the gate oxide. The total gate capacitance is calculated as [19]

$$
C_{G G}=C_{G S}+C_{G D}+C_{G B}=C_{o x} W L+2 C_{o x} x_{d} W
$$

All capacitances connected to the gate of the MOSFET should be modeled for delay calculations.

\subsubsection{Junction Capacitance}

The source and the drain diffusions into the substrate create reversed p-n junctions, which contribute to the junction-drain $\left(C_{J D}\right)$, and the junction-source $\left(C_{J S}\right)$ capacitances. Each junction capacitance consists of a bottom-plate and a side-wall capacitance. The bottomplate capacitance is presented as [19]:

$$
C_{\text {bottom }}=C_{j} W L_{s}
$$

where $C_{j}$ is the junction capacitance per unit area, and $L s$ is the side-wall length. The sidewall capacitance is expressed as:

$$
C_{s W}=C_{j s w}\left(W+2 L_{s}\right)
$$

where $C_{j s w}$ is the capacitance per unit perimeter. Note that the diffusion-to-channel capacitance is ignored. The total diffusion capacitance is then:

$$
C_{\text {bottom }}+C_{s W}=C_{j} W L_{s}+C_{j s w}\left(W+2 L_{s}\right)
$$

Considering Equations (2-14) and (2-17), a linear relationship exists between the total capacitance of a MOSFET with its width.

\subsection{Circuit Operation Robustness}

When operating in the subthreshold region, the channel under the gate is not inverted. As a result, the tolerance of the transistor when it comes to PVT variations and mismatch are 
different when operating in the superthreshold region [13]. An increase or decrease in the drain current changes the drive of the transistor, making blocks and gates imbalanced. The exponential relationship between the subthreshold drain current and the threshold voltage increases the effect of threshold voltage variations [3] compared to superthreshold operations. These different variations are examined in this section.

\subsubsection{Supply Voltage}

Systems are designed to operate at a nominal supply voltage. However, this voltage may change for reasons, such as, tolerances of the voltage regulators, $I \times R$ drops along supply rails [21]. The system designer may trade off power supply noise against resources assigned to power supply regulation and distribution; mostly, the supply is determined at $\pm 10 \%$ around the nominal value [21].

\subsubsection{Temperature Variations}

Temperature variations affect CMOS circuits for two reasons: Firstly, as the temperature rises, the mobility factor decreases, which gives a lower drain current and an increased CMOS gate delay. In addition, the threshold voltage also decreases as the temperature rises, giving a higher Drain Current and a decreased CMOS gate delay. This can be seen in Equation (2-18) and (2-19) [36],

$$
\begin{gathered}
\mu(T)=\mu\left(T_{0}\right)\left(\frac{T}{T_{0}}\right)^{-M} \\
V_{t h}(T)=V_{t h}\left(T_{0}\right)-K T
\end{gathered}
$$

where $\mu$ mobility, $\mu\left(T_{0}\right)$ is mobility at room temperature, $T_{0}$ is room temperature, $K$ is the threshold voltage coefficient and $M$ is the mobility temperature exponent.

\subsubsection{Process Variations}

Devices and interconnect have variations in film thickness, lateral dimension, and doping concentrations. Consequently, one transistor might have a different threshold voltage than the neighboring one, because of the random number of dopant atoms. For most devices, 
the most significant variations are channel length and threshold voltage [21]. Photolithography proximity effects, deviation in optics, and plasma etch dependencies cause channel length variations. Doping concentrations, and annealing effects, mobile charge in the gate oxide, and dopant variation change threshold voltage. For interconnect, the most significant variations are line width and spacing, metal and dielectric thickness, and contact resistance [21]. Process variations can be split into global process variations and local process variations [3]:

\section{- Global Process Variations}

Variations that are equal over the die, like wafer-to-wafer misalignments or processing temperatures. These variations normally affect all transistors in the system to the same degree. However, some parts of the circuit can be more vulnerable to process variations and can cause threshold voltage variations [3].

\section{- Local Process Variations}

These variations only affect parts of the die or circuit. The local process variations can consist of both systematic and random components. These can typically be aberrations in the processing equipment, which can produce systematic variations. In addition, they may affect the placement and number of dopant atoms in the device [3].

\subsubsection{Design Corners}

Process variation affects are exposed when there are two types of transistors with different speeds. When these processing variations are merged with the environmental fluctuations,

they are referred to as process corners. The term corner notes to an imaginary box that surrounds the guaranteed performance of circuits, as shown in Figure 2. They are named typical-typical, fast-fast, slow-slow, fast-slow, slow-fast. Integrated circuits are designed to meet a timing specification for typical processing. The faster parts are allowed for higher frequency and slower parts are allowed for lower frequency [21]. 


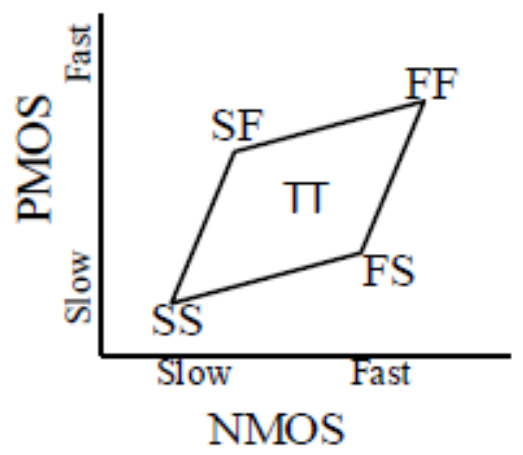

Figure 2 Process corners.

\subsection{Effect of Channel Length Variations on Threshold Voltage}

In short channel devices, $V_{\text {th }}$ has an exponential dependence on the channel length $L$ due to charge-sharing and DIBL effects expressed as follows [37]

$$
V_{t h} \approx V_{t h 0}-\left(\zeta+\eta V_{d s}\right) \exp ^{-\frac{L}{\lambda}}
$$

where $V_{t h 0}$ is the long channel threshold voltage, $\zeta$ is the charge-sharing coefficient, $\lambda$ is the characteristic length, and $\eta$ is the DIBL coefficient. As a result, a slight variation in $L$ introduces a large variation in $V_{t h}$ due to the exponential dependence described in Equation (2-20).

\subsection{Sequential Computing}

A major part of digital VLSI systems is designed as a clocked sequential system, using a global clock for the purpose of synchronization. This clock triggers the registers all over the system at the same time. A sequencing element, connected to the clock, is used to synchronize data. Combinational logic is placed between the sequencing elements, as illustrated in Figure 3. The purpose of a sequencing element is to enforce the sequence, to distinguish the current token from the previous or next token [19].

The two most commonly used sequencing elements are flip-flops and latches. Flipflops and latches can be separated into how the output signal is changed when the input 
signal changes. When the input signal flows directly through to the output, the element is said to be transparent [19]. Latches are transparent while the clock signal is high, while flip-flops are not transparent at any time.

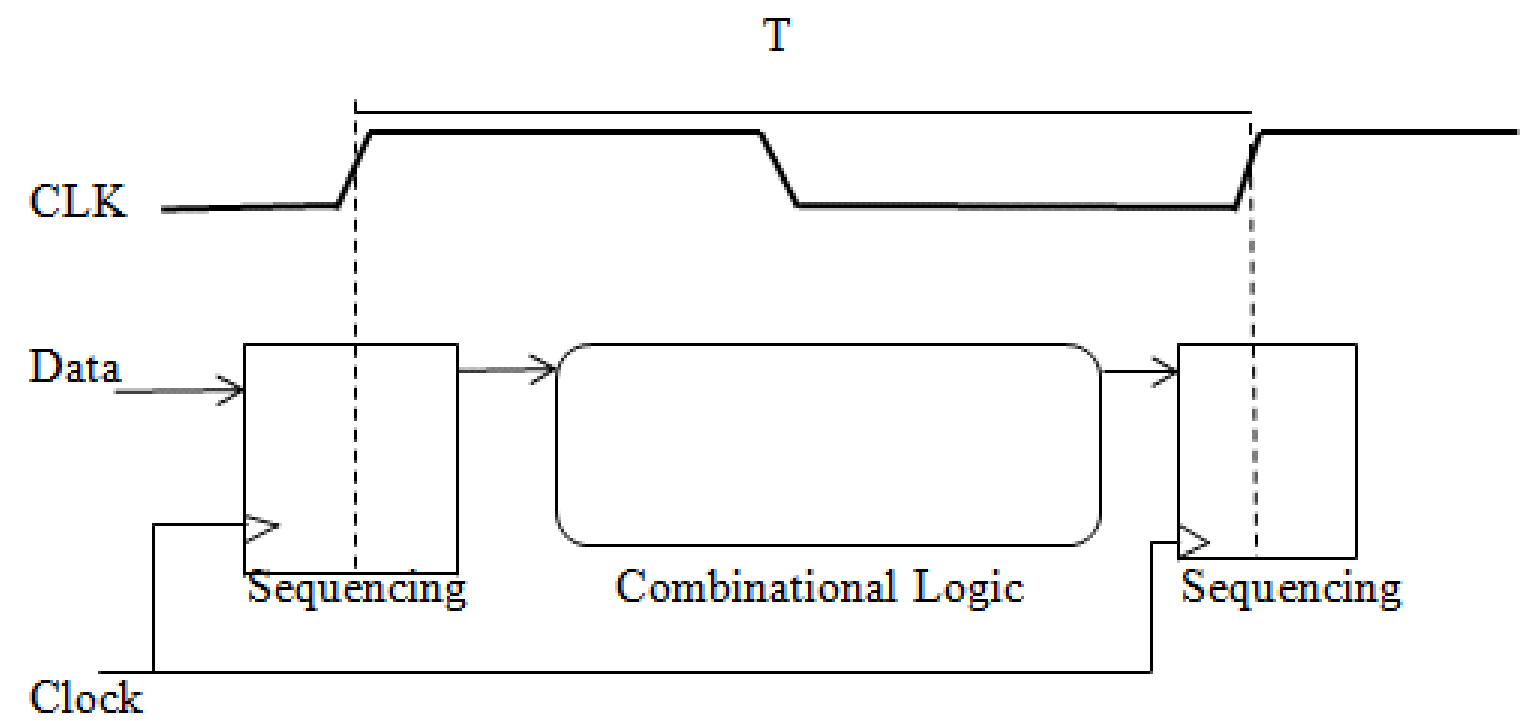

Figure 3 Clocked sequential system.

\subsubsection{Flip-Flops}

Most high-performance digital designs today utilize a synchronous clock to order events [38]. The principle of synchronization is easy from the perspective of system design. However, ordering all events in a high performance design in a synchronous fashion requires generation and distribution of clock signals at multi GHz clock frequencies, which is challenging. Therefore, it is very important to design the flip-flops so that they can be optimized for the yield and reliability constraints.

Synchronization circuits such as latches and flip-flops constitute the clocked registers that synchronize the data flow in a VLSI circuit. Hence, flip-flops and latches are among the most important circuit blocks in a digital synchronous chip design. Ideally, timing circuits like flip-flops and latches should add as little latency as possible, and have low 
power dissipation. In practice, however, clocked registers can actually consume a substantial fraction of the clock-cycle period, and dissipate a considerable portion of the total power [19] [38].

Flip-flops are an important building block in modern digital VLSI systems. Some of the major usage areas of flip-flops are in registers, pipelines and state machines, ensuring sequencing of data. A flip-flop reads an input value, saves it for some time and then writes the stored value at the output. This procedure is completed, regardless of the subsequent change in the element's input value.

Based on the comparison of the power breakdown for different elements in VLSI chips, latches and flip-flops are the major sources of the power consumption in synchronous systems. Flip-flops have a direct impact on power consumption and speed of VLSI systems. When estimating the power dissipation of a system, flip-flops may be a major power consumption component [39].

The basic types of flip flops include set-or-reset (SR) Flip-Flop, D Flip-Flop, JK Flip-Flop, and T Flip-Flop. The D flip flop is most widely used as they have the capability to be forced to the set or reset state (ignoring the D and clock inputs), much like an SR Flip-Flop. In addition, usually the illegal $\mathrm{S}=\mathrm{R}=1$ condition is resolved in D-type Flip-Flops [19].

\subsubsection{Common Flip-Flop Topologies}

A large number of flip-flop circuits exist, which can be classified in three categories: latch pairs, pulsed latches, and sense-amplifier based flip-flops [38] [40]. The most common approach for constructing an edge-triggered register is to use two latches configuration, as shown in Figure 4 [38]. The register consists of cascading a negative latch (master stage) with a positive latch (slave stage). A multiplexer-based latch is used in this particular implementation, although any latch could be used. On the low phase of the clock, the first stage is transparent, and the D input is passed to the second stage output, $\mathrm{Q}_{1}$. During this period, the second stage is in the hold mode, keeping its previous value using feedback. On

the rising edge of the clock, the first stage stops sampling the input, while the second stage starts to sample. During the high phase of the clock, the second stage samples the output 
of the master stage $\left(\mathrm{Q}_{1}\right)$, while the first stage remains in a holding mode. Since $\mathrm{Q}_{1}$ is constant during the high phase of the clock, the output Q makes only one transition per cycle. The value of $\mathrm{Q}$ becomes the value of $\mathrm{D}$ right before the rising edge of the clock, achieving a positive edge-triggered effect. A negative edge-triggered register can be constructed using the same principle by simply switching the order of the positive and negative latches [19].

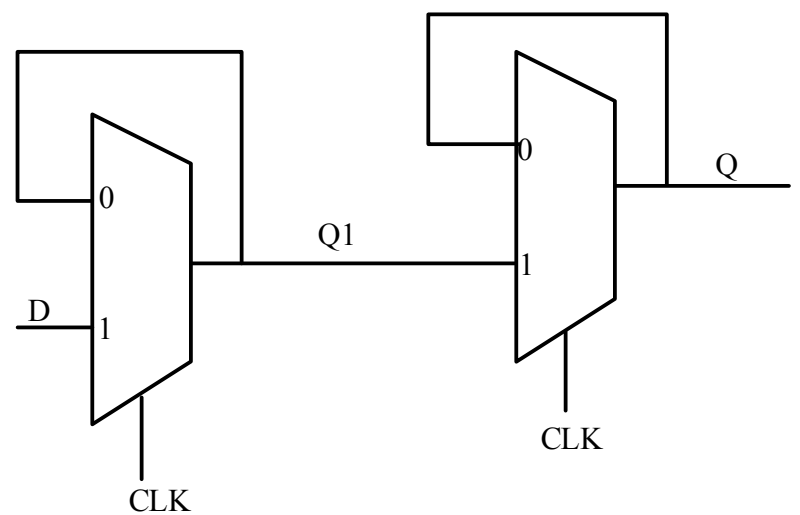

Figure 4 Positive edge-triggered register based on two latches configuration.

A transmission gate flip-flop, which is another example of an edge-triggered flipflop, is shown in Figure 5 [40]. The setup time of this flip-flop is mainly determined by the propagation delay of the first latch. In addition, the output latency is determined by the propagation delay through the second latch, resulting in a quite large latency delay [19] [40]. 


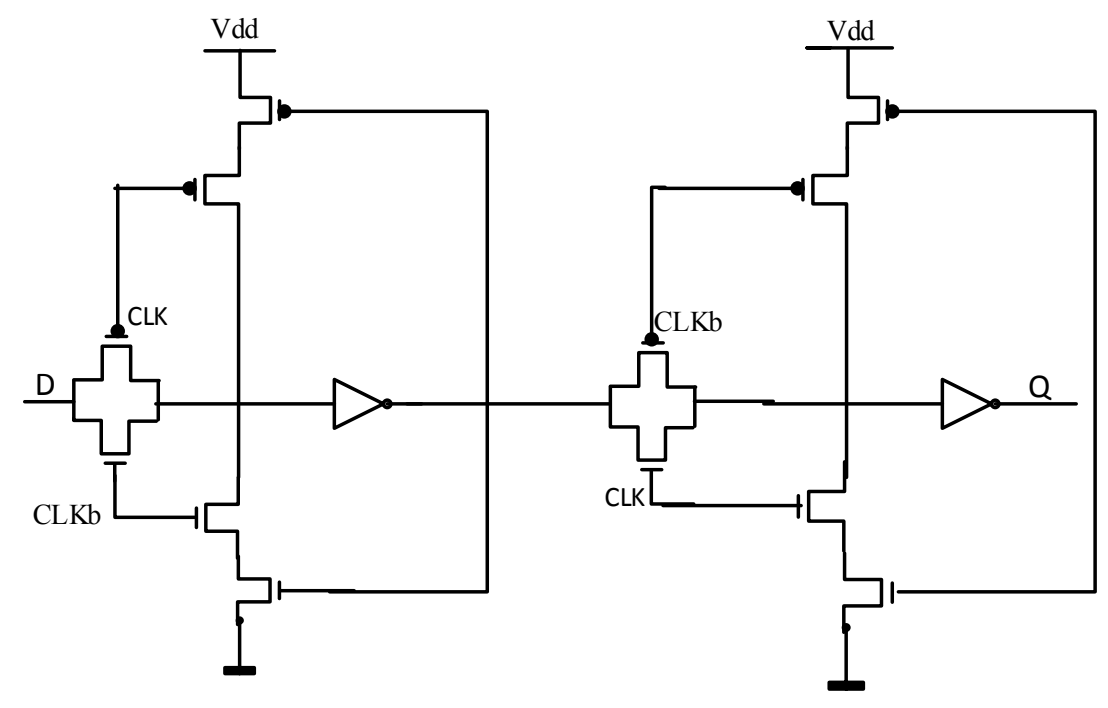

Figure 5 Transmission gate flip-flop structure.

\subsubsection{Flip-Flop Timing characteristic}

A timing diagram of a positive edge-triggered flip-flop is shown in Figure 6. All timing relations for the edge-triggered flip-flop are referred only to the sampling clock edge. The timing relations for an edge-triggered flip-flop are defined by essentially four different delays as stated below [38]. 


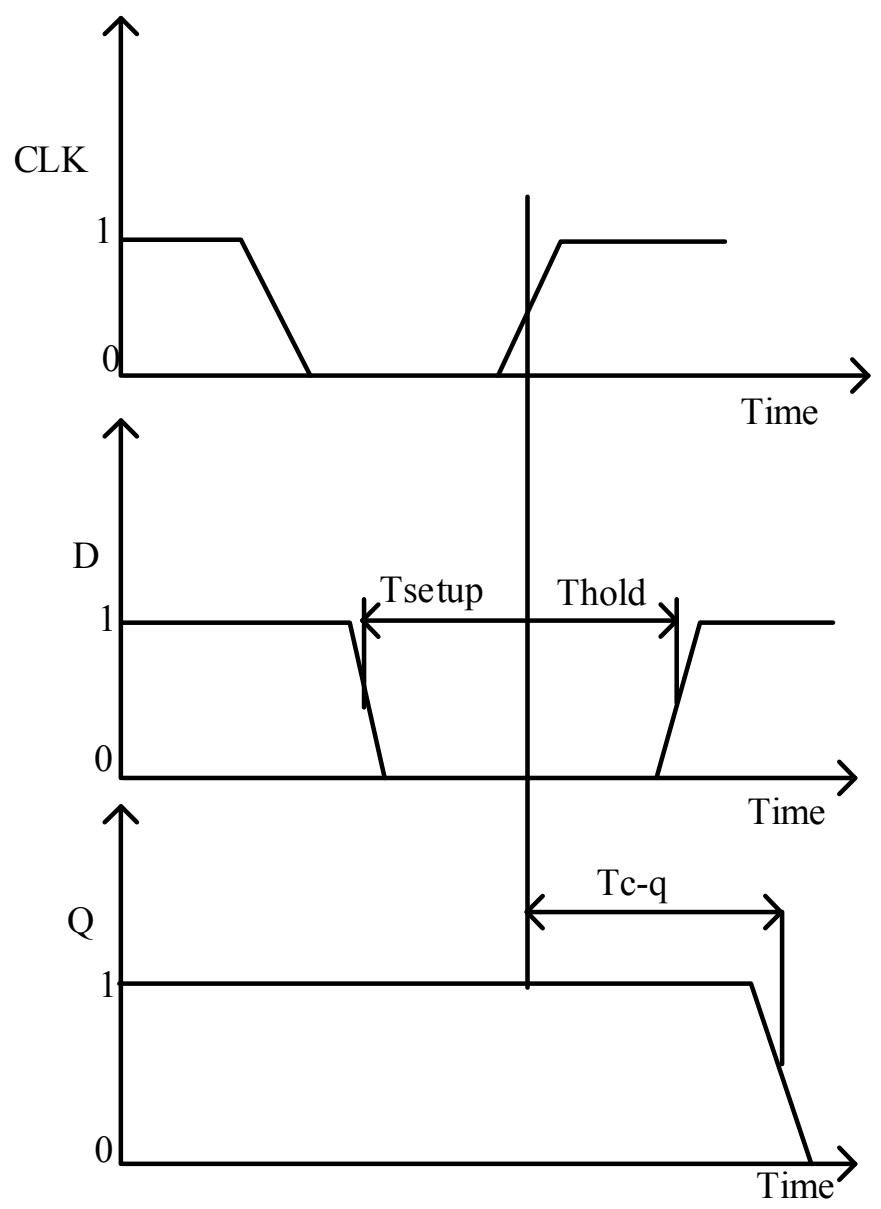

Figure 6 Flip-Flop timing diagram.

- $\quad$ Setup time $\left(T_{\text {setup }}\right)$ :

The minimum time that the input data should be available before the clock sampling edge arrival.

- Hold time $\left(T_{\text {hold }}\right)$ :

The minimum time that the input data should be available after the clock sampling edge arrival.

\section{- Clock-to-output delay $\left(T_{c-q}\right)$ :}

The delay from the sampling clock edge to the time at which the latched data is valid at the output. 


\section{- Total delay $(T)$ :}

The delay from a transition of the input data to the time at which the latched data is valid at the output. This delay can be determined as the sum of the setup time and the clock-to-output delay.

\subsubsection{Propagation Delay $\left(t_{p}\right)$}

The delay of a transistor can be estimated as the time is taken to charge and discharge of the output node through either the PMOS or NMOS transistor [21]:

$$
t_{p}=\frac{C V_{d d}}{2 I_{o n}}
$$

By substituting Equation (2-3) in Equation (2-18):

$$
t_{p}=\frac{C V_{d d}}{I_{0} \exp \frac{V_{d d^{-V} t h}}{n V_{t h}}}
$$

Therefore, decreasing $I_{o n}$ leads to exponential increase in delay at lower $V_{d d}$.

\subsubsection{Energy Consumption}

When output of an inverter makes a transition from " 0 " to $V_{d d}$, the dynamic energy drawn from the power supply in one cycle is expressed [21]:

$$
E_{d}=C V^{2} d d
$$

For this transition, the stored energy in the load capacitance is

$$
E_{t}=\frac{1}{2} C V^{2} d d
$$

where $C$ is output capacitance, and $V_{d d}$ is supply voltage.

Equation (2-24) Shows 50\% of the total energy drawn from the supply is consumed by the PMOS transistor. Furthermore, transition from $V_{d d}$ to " 0 ", the stored energy in the 
capacitor is consumed by the NMOS transistor and no energy is drawn from the power supply.

The static energy in one cycle is other component of consumed energy which is expressed as

$$
E_{s t}=I_{l e a k} V_{d d} t_{p}
$$

where $t_{p}$ is the propagation delay, or the time required to complete one computation and $I_{\text {leak }}$ is the total leakage current. By substituting Equation (2-21) in Equation (2-25), the leakage energy becomes:

$$
E_{\text {st }}=I_{\text {leak }} V_{d d} \frac{C V_{d d}}{2 I_{\text {onav }}}
$$

Hence, the total energy per cycle is expressed as

$$
\begin{aligned}
& E_{t}=E_{d}+E_{s t} \\
& E_{t}=C V_{d d}^{2}\left(1+\frac{C V_{d d}}{2 I_{\text {onav }}}\right)
\end{aligned}
$$

Equation (2-28) shows a quadratic relation between the total consumed energy and the supply voltage. Hence, decreasing $V_{d d}$ decreases the total energy quadratically. However, decreasing $V_{d d}$ decreases $I_{o n a v}$ too. Thus, it is predictable that consumed energy shows a minimum point with respect to $V_{d d}$. This is later shown in the upcoming chapters in more detail.

\subsection{Summary}

In this chapter, we presented a detailed study of MOSFET behavior operating in subthreshold region. Furthermore, the importance of flip-flops in sequential synchronized digital systems and the related theoretical background were reviewed. This background 
knowledge is used in the next chapters to compare and make reliability enhancements for different subthreshold flip-flop topologies using sizing and device methods. 


\section{Chapter 3. Device Characterization}

The main characteristics of an MOS transistor that need to be studied are: the threshold voltage $\left(V_{t h}\right)$, transistor's ON current $\left(I_{O N}\right)$ and OFF current $\left(I_{O F F}\right)$, and capacitances for better understanding of transistor sizing. In this chapter the effect of transistor's channel length $(L)$ and width $(W)$ manipulations are studied within these parameters. Note that $L$ and $W$ are the two major aspects that can be altered by a circuit designer at the schematic and layout levels. Three different transistor types are considered here: high threshold (hvt), standard threshold (svt), and low threshold (lvt) voltage for NMOS and PMOS transistors in. The CAD tool used is Cadence, the simulation engine is Spectre, the transistor models are based on BSIM4, and the technology kit is TSMC $65 \mathrm{~nm}$ Low power (LP) CMOS.

\subsection{Threshold Voltage Variations}

Changes in $V_{\text {th }}$ affects the delay through $I_{O N}$, and affects the dynamic energy consumption if the circuit is to operate at its maximum frequency. It also influences IOFF and, hence, the static energy consumption of a circuit. Lower threshold voltage increases the leakage current; in the case of subthreshold circuits, this includes both $I_{O N}$ and $I_{O F F}$.

The threshold voltage of a transistor may change in a number of ways. One is through the body effect. If the source-body junction of an MOSFET is reverse-biased (forward-biased), that is, having positive (negative) $V_{s b}$ in NMOS and negative (positive) $V_{s b}$ in PMOS transistors, the absolute value of $V_{t h}$ increases (decreases).

The second way is through Drain Induced Barrier Lowering (DIBL) phenomenon. The extent of the DIBL effect depends on the gate voltage and drain voltage [19]. Figure 7 shows the DIBL effect on an NMOS transistor in the $65 \mathrm{~nm}$ bulk CMOS technology. As $V_{d s}$ increases, $V_{t h}$ decreases and, in turn, the current increases, as noticeable by the curve current shifting to the left. 


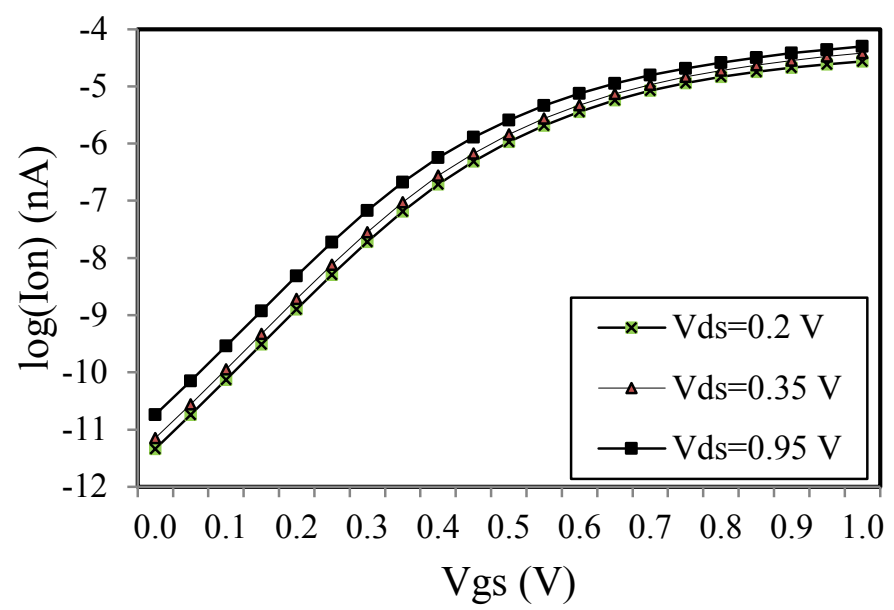

Figure $7 \log \left(I_{o n}\right)$ as a function of $V_{g s}$ for a minimum size hvt-NMOS transistor in the $65 \mathrm{~nm}$ technology.

A third way of changing $V_{t h}$ is through the so-called reverse-short-channel effect (RSCE) [21]. Figure 8 and Figure 9 show NMOS and PMOS transistors' threshold voltage variations versus $L$ in the high, standard, and low threshold voltage devices. As the channel length is reduced in all cases, the absolute value of threshold voltage rolls-up.

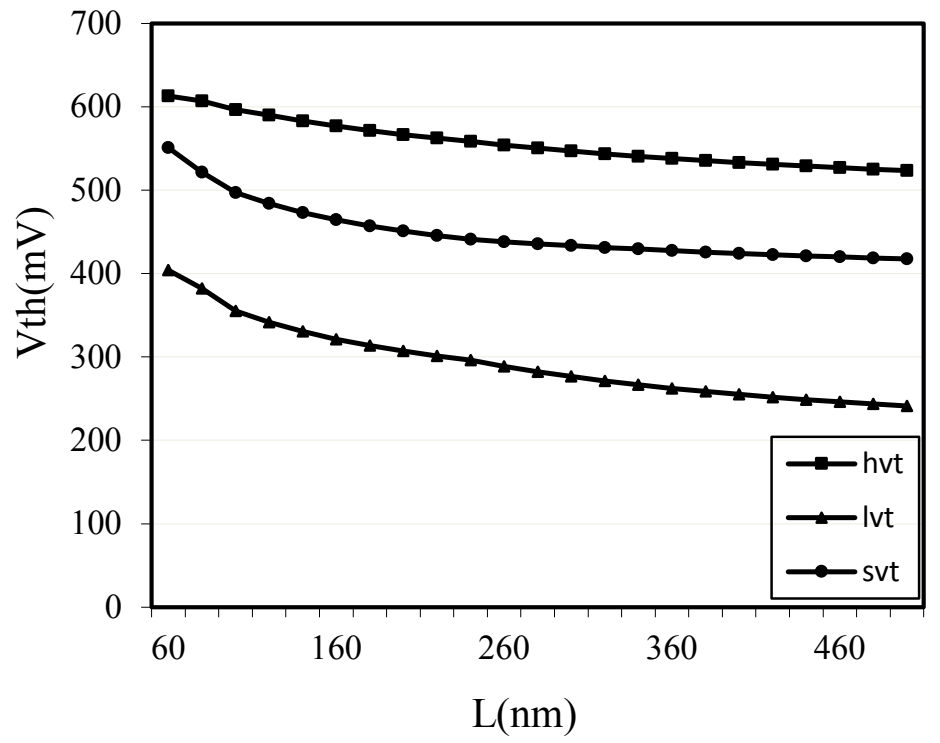

Figure $8 V_{t h}$ versus $L\left(W=W_{\min }=120 \mathrm{~nm}, V_{d d}=200 \mathrm{mV}, 65 \mathrm{~nm}\right.$ process, NMOS). 


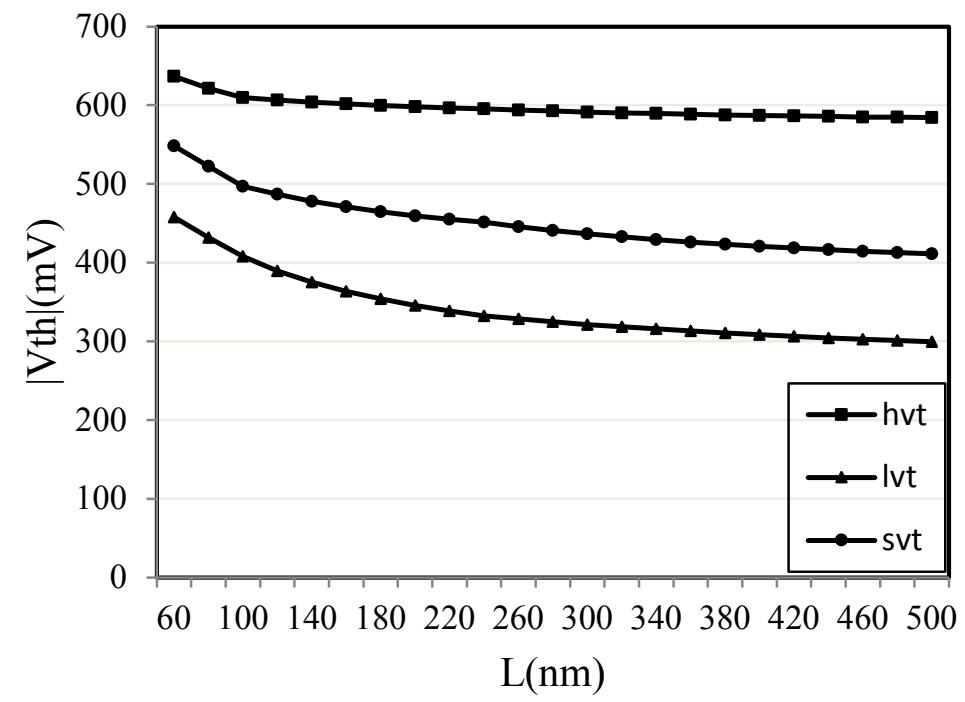

Figure $9 V_{t h}$ versus $L\left(\mathrm{~W}=\mathrm{W}_{\min }=120 \mathrm{~nm}, V_{\mathrm{dd}}=200 \mathrm{mV}, 65 \mathrm{~nm}\right.$ process, $\left.P M O S\right)$.

A fourth way of affecting $V_{t h}$ is through the so-called Inverse-Narrow-Width-Effect (INWE) [21]. This effect is responsible for lowering $V_{t h}$ in transistors with small $W$, i.e., narrow transistors. Figure 10 and Figure 11 show the variations in the threshold voltage versus the channel width for NMOS and PMOS transistors. The INWE is not evident is all technologies neither in all transistor types. As Figure 11 shows, in the $65 \mathrm{~nm}$ technology, PMOS transistors are barely affected by the INWE.

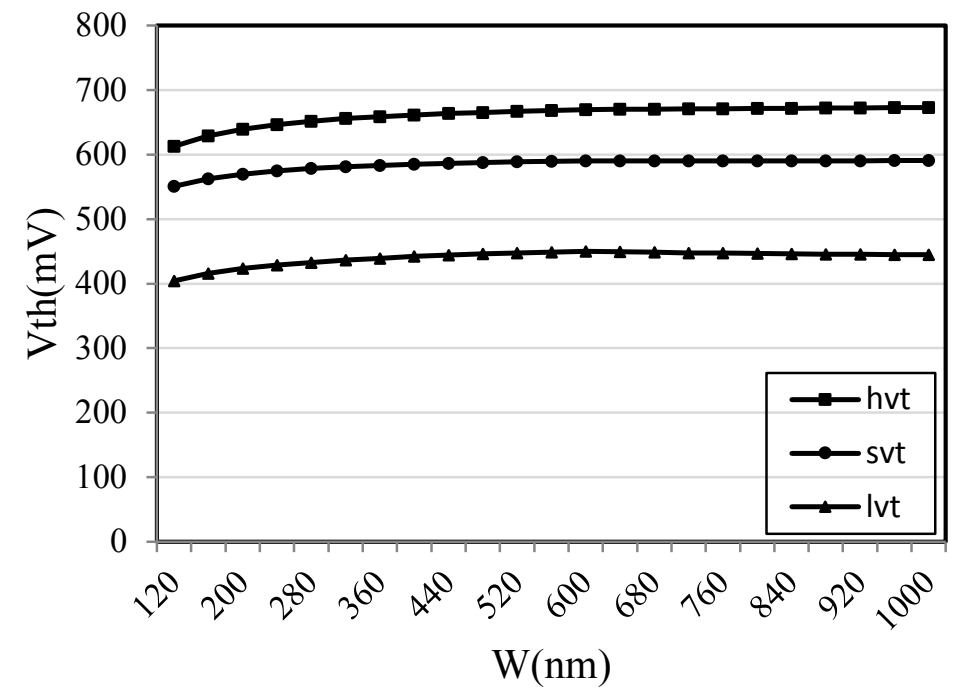

Figure $10 V_{\text {th }}$ versus $W\left(L=L_{m i n}=60 \mathrm{~nm}, V_{d d}=200 \mathrm{mV}, 65 \mathrm{~nm}\right.$ process, NMOS $)$. 


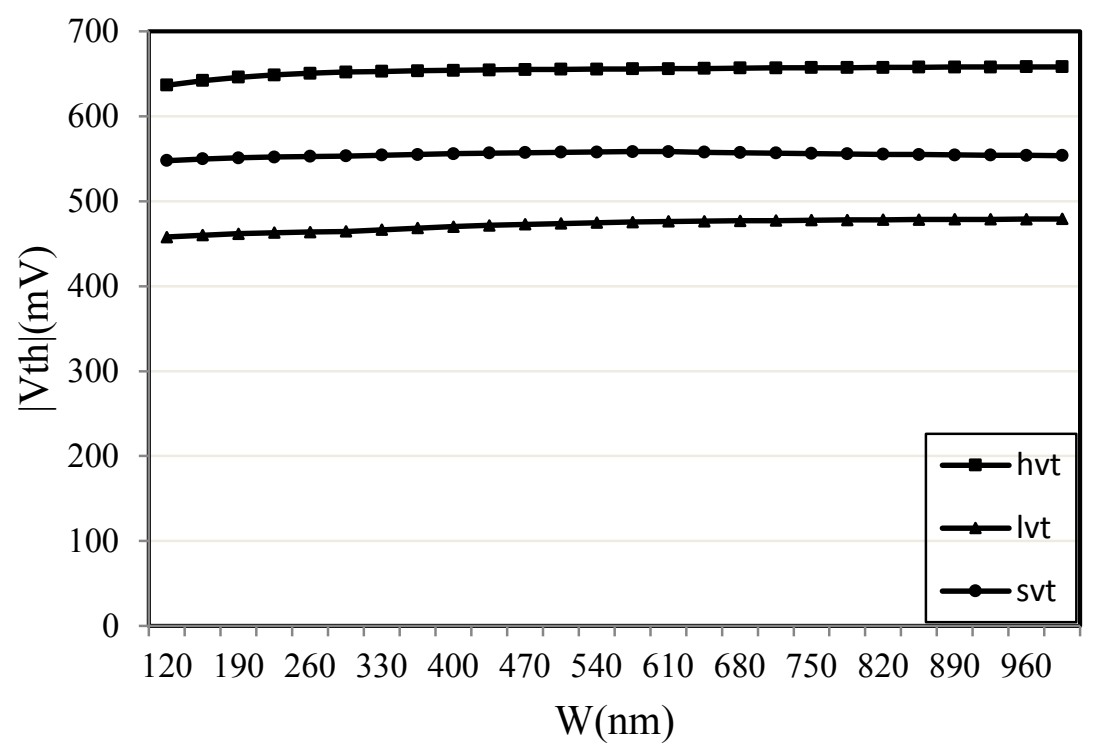

Figure $11 V_{t h}$ versus $W\left(L=L_{m i n}=60 \mathrm{~nm}, V_{d d}=200 \mathrm{mV}, 65 \mathrm{~nm}\right.$ process, PMOS $)$.

\subsection{Current Behaviour}

In the subthreshold region, because the current is exponentially related to the threshold voltage, the current behavior is not as simple as in the case of the superthreshold region.

From Figure 8 and Figure 9, it is clear that as $L$ increases, $V_{t h}$ decreases. On the other hand, when $e^{\frac{v_{g s}-V_{t h}}{n V_{T}}}$ increases, $1 / L$ decreases. Since the subthreshold current is proportional to $1 / L$ times $e^{\frac{V g s-V_{t h}}{n V_{T}}}$, it is difficult to predict the behaviour of the subthreshold current as a function of $L$. The same discussion is valid in relation to $W$.

To measure the ON and and OFF currents, the simulation test benches for NMOS and PMOS transistors are arranged as shown in Figure 12. Further, NMOS and PMOS gate current and junction current test benches are shown in Figure 13 and Figure 14 respectively. The general concepts of leakage currents $\left(I_{G}, I_{s u b}, I_{j u n c}\right)$ were presented in section 2.1.3. As shown in Table 2, the simulation results show that $I_{\text {sub }}$ is much larger than $I_{G}$ and $I_{\text {junc }}$. Hence, the focus will be on $I_{s u b}$ as the main source of the leakage current by far. 


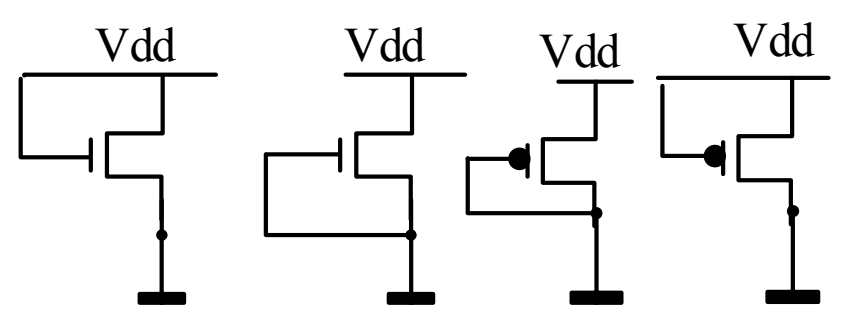

(a)

(b)

(c)

(d)

Figure 12 Test Benches for measuring (a) NMOS ON current, (b) NMOS OFF current, (c) PMOS ON current, and (d) PMOS OFF current.

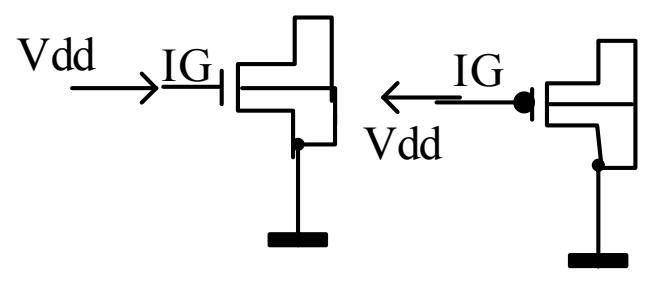

(a)

(b)

Figure 13 Test benches used for gate currents measurement (a) NMOS gate current (b) PMOS gate current.

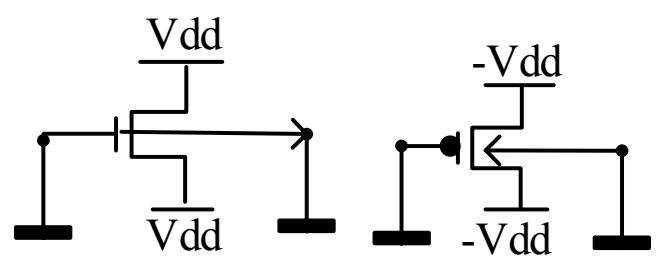

(a)

(b)

Figure 14 Test benches used for junction currents measurement (a) NMOS junction current (b) PMOS junction current. 
Table 2 NMOS and PMOS transistors leakage currents $\left(L=L_{\min }=60 \mathrm{~nm}, W=W_{\min }=120 \mathrm{~nm}, V_{d d}=\right.$ $200 \mathrm{mV}, 65 \mathrm{~nm}$ process).

\begin{tabular}{|l|l|l|l|}
\hline & $\boldsymbol{I}_{\boldsymbol{G}}(\mathbf{f A})$ & $\mathbf{I}_{\text {sub }}(\mathbf{p A})$ & $\mathbf{I}_{\mathbf{j u n c}} \mathbf{( z A )}$ \\
\hline hvt-NMOS & 1.277 & 0.81 & 192 \\
\hline hvt-PMOS & 0.85 & 0.34 & 77 \\
\hline svt-NMOS & 1.56 & 4.592 & 186 \\
\hline svt-PMOS & 1.2 & 2.46 & 112 \\
\hline lvt-NMOS & 4.673 & 35.62 & 170 \\
\hline lvt-PMOS & 2.81 & 7.84 & 82 \\
\hline
\end{tabular}

In the subthreshold region of operations, both $I_{O N}$ and $I_{O F F}$ are expressed by Equation (2-3), except that to calculate $I_{O F F}$, the gate-source voltage $V_{g s}$ should be set to " 0 ". Hence, it seems that the OFF and ON-currents show the same behavior with respect to the changes in a transistor size. The ON-current, OFF-current and threshold voltage values for minimum-size (hvt, svt, and lvt) NMOS and (hvt, svt, and lvt) PMOS transistors in the TSMC 65 nm LP CMOS technology are listed in Table 3.

Table $3 I_{O N}, I_{O F F}$, and $V_{t h}$ of devices $\left(\left(L=L_{\min }=60 \mathrm{~nm}, W=W_{\min }=120 \mathrm{~nm}, V_{d d}=200 \mathrm{mV}, T=27^{\circ} \mathrm{C}, 65\right.\right.$ nm process).

\begin{tabular}{|l|l|l|c|}
\hline & Ion $(\mathbf{n A})$ & IoFF $(\mathbf{p A})$ & $\boldsymbol{V}_{\boldsymbol{t} \boldsymbol{}}(\mathbf{m V})$ \\
\hline hvt-NMOS & 0.12 & 0.81 & 589.8 \\
\hline hvt-PMOS & 0.03 & 0.34 & -636.7 \\
\hline svt-NMOS & 1.275 & 4.592 & 483.8 \\
\hline svt-PMOS & 0.45 & 2.46 & -548.1 \\
\hline lvt-NMOS & 11.01 & 35.62 & 341.6 \\
\hline lvt-PMOS & 1.87 & 7.84 & -457.9 \\
\hline
\end{tabular}




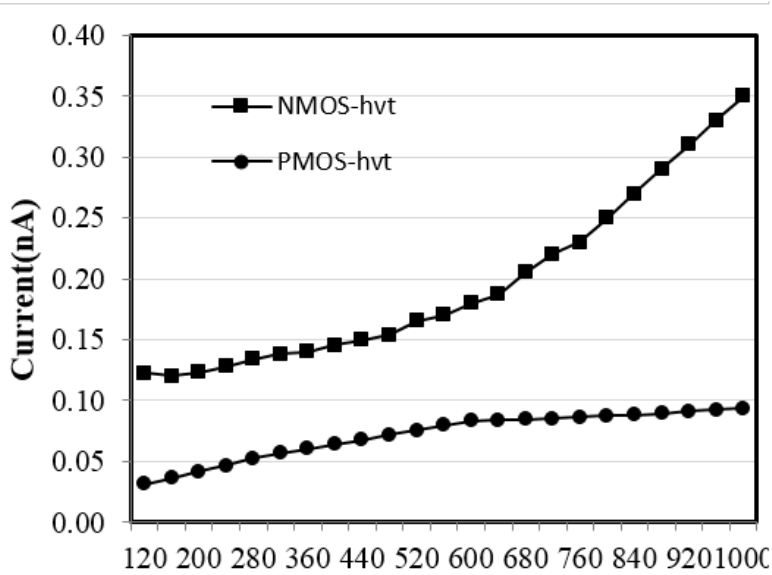

$120200280360440520600680760840920100 c$

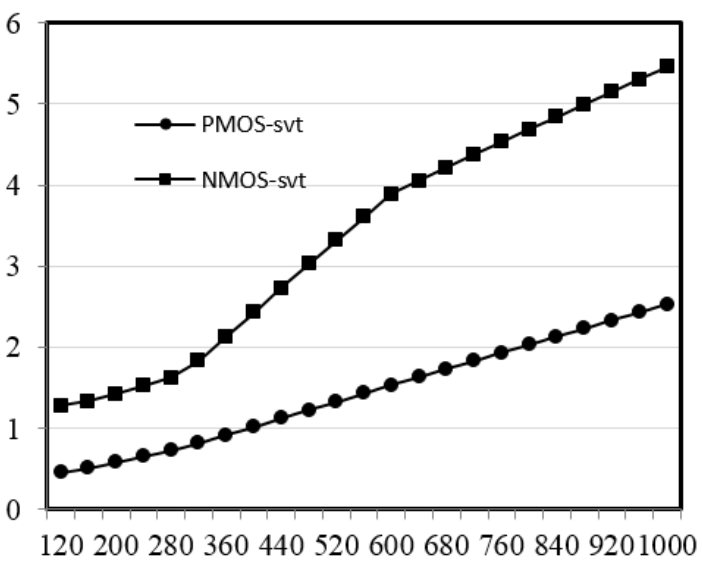

$\mathbf{W}(\mathbf{n m})$

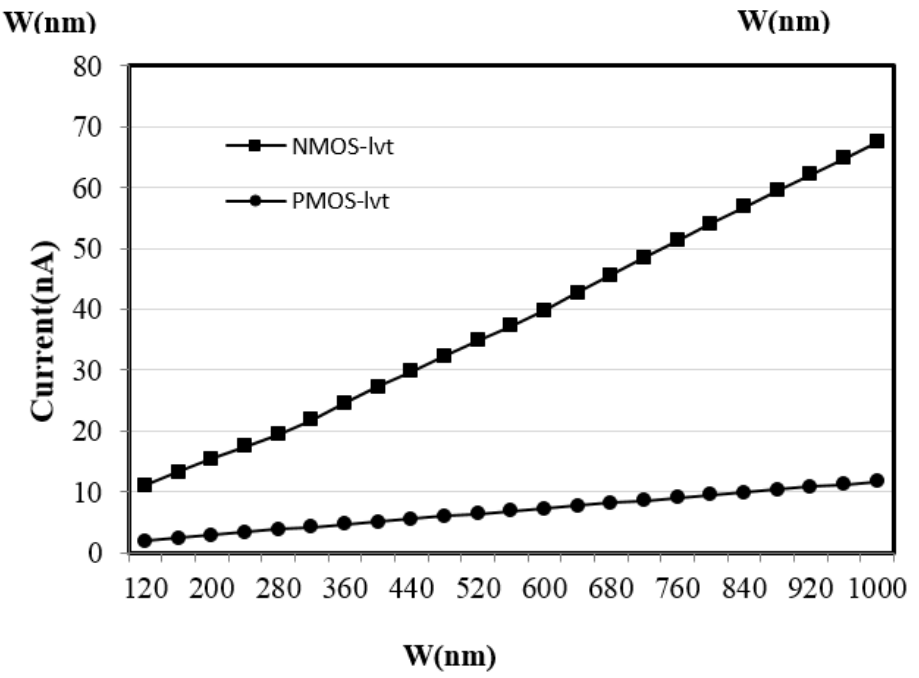

Figure $15 I_{\text {on }}$ versus $W\left(L=L_{\min }=60 \mathrm{~nm}, V_{d d}=200 \mathrm{mV}, 65 \mathrm{~nm}\right.$ process $)$.

In Figure 15 the ON currents are plotted versus the channel width for (hvt, svt, and lvt) NMOS and (hvt, svt, and lvt) PMOS transistors, with shortest channel length, in the same technology. Figure 16 shows the same current parameters versus the channel length for NMOS and PMOS transistors with minimum width. As illustrated in these figures, the behaviour of the current in the subthreshold region with respect to $W$ and $L$ varies from one transistor type to another in the TSMC $65 \mathrm{~nm}$ LP CMOS. In all cases $I_{O N}$ increases with increasing $W$, but with increasing $L$ it initially drops then stabilizes at larger $L$. The currents in the lvt-NMOS and lvt-PMOS transistors, exceptionally show initial rises and maximum points with respect to $L$. 

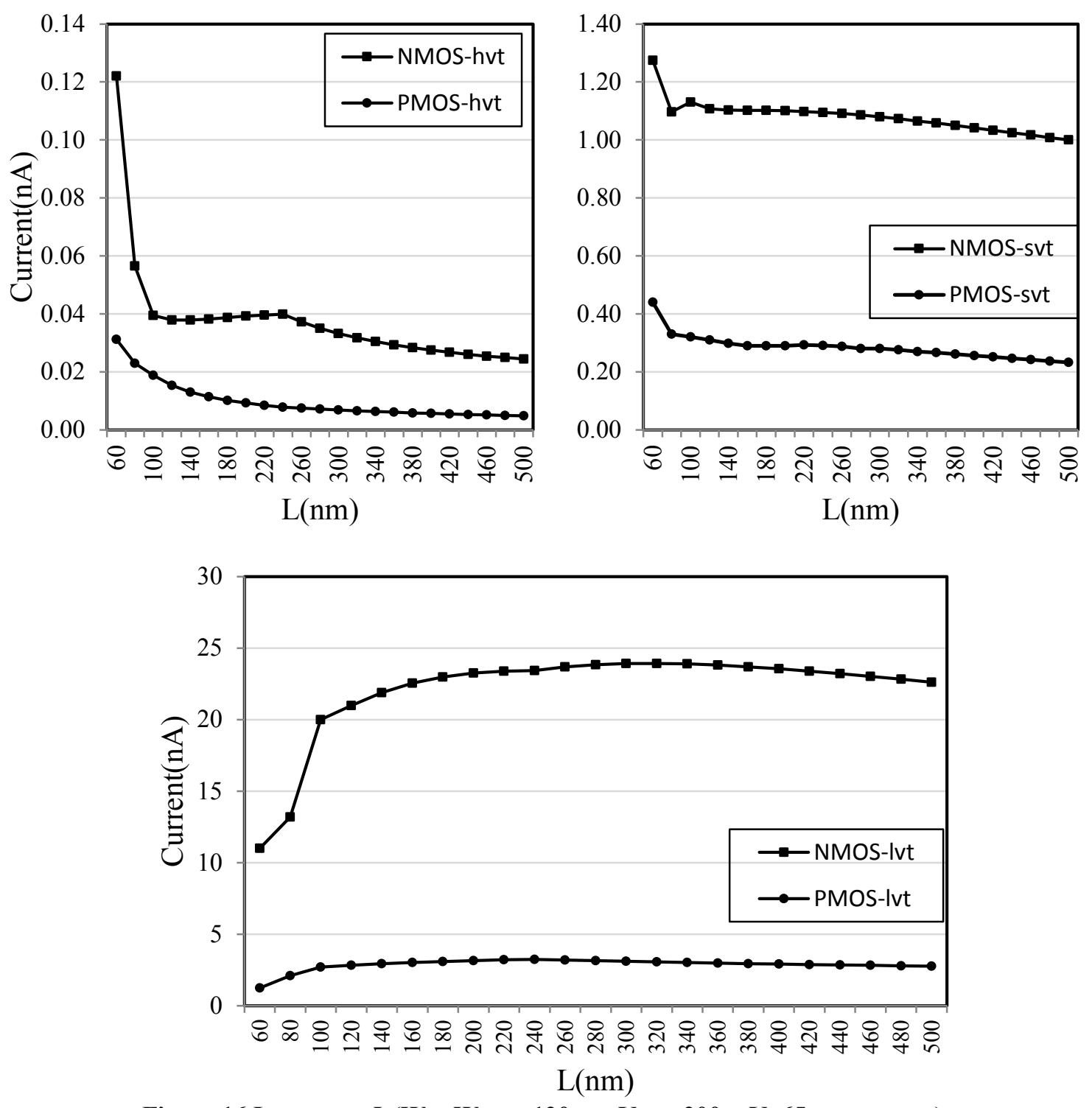

Figure $16 I_{0 N}$ versus $L\left(W=W_{\min }=120 \mathrm{~nm}, V_{d d}=200 \mathrm{mV}, 65 \mathrm{~nm}\right.$ process $)$.

The OFF currents, as expected, behave similar to the ON currents with respect to $W$ and $L$, while being about three orders of magnitude smaller. This suggests $I_{O N}$ over $I_{O F F}$ ratios of around 1000 that seems large enough for noise tolerance. The OFF currents versus $W$ are shown in Figure 17, and in Figure 18 versus $L$. 

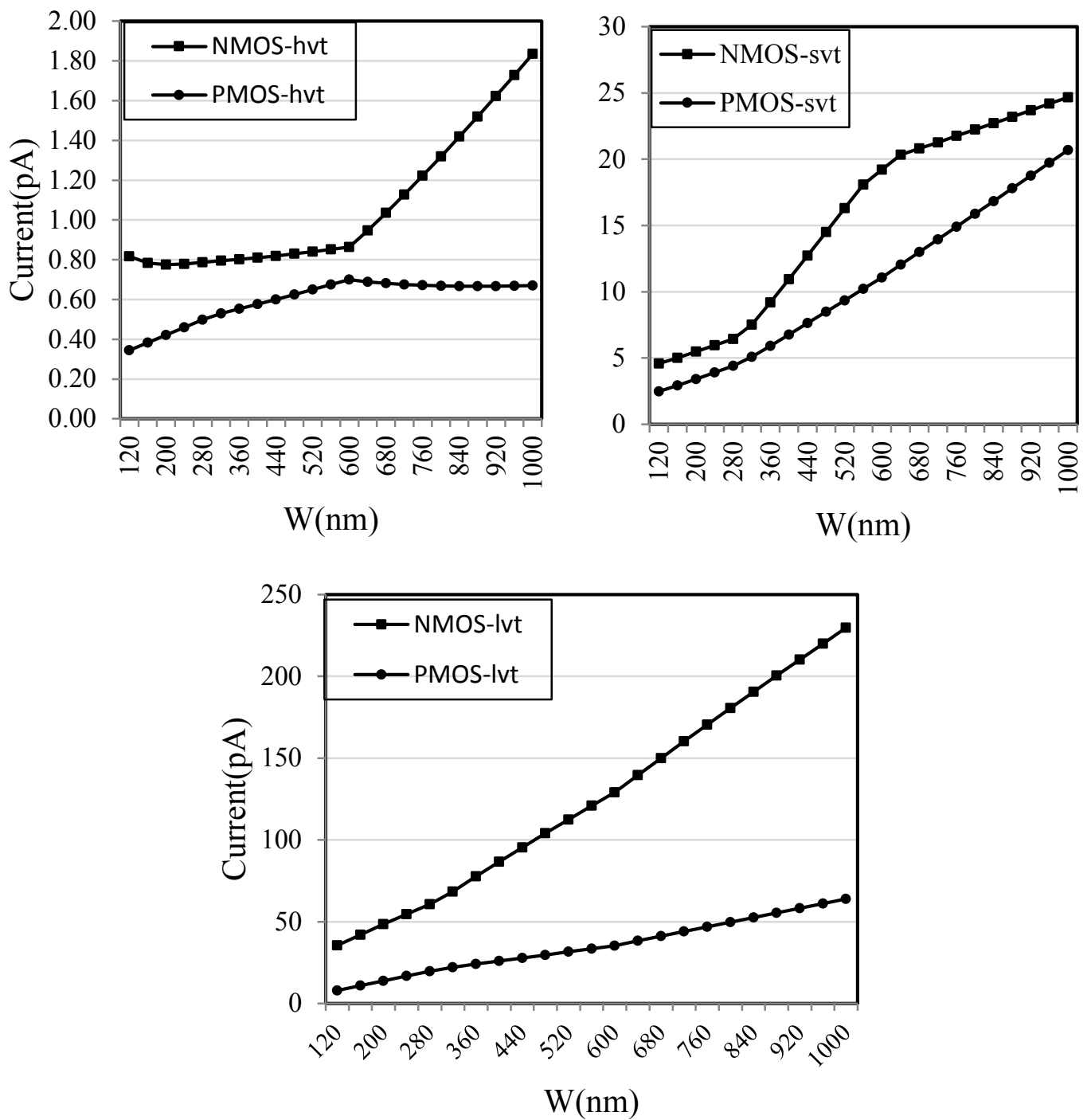

Figure 17 Ioff versus $W\left(L=L_{\min }=60 \mathrm{~nm}, V_{d d}=200 \mathrm{mV}, 65 \mathrm{~nm}\right.$ process $)$. 

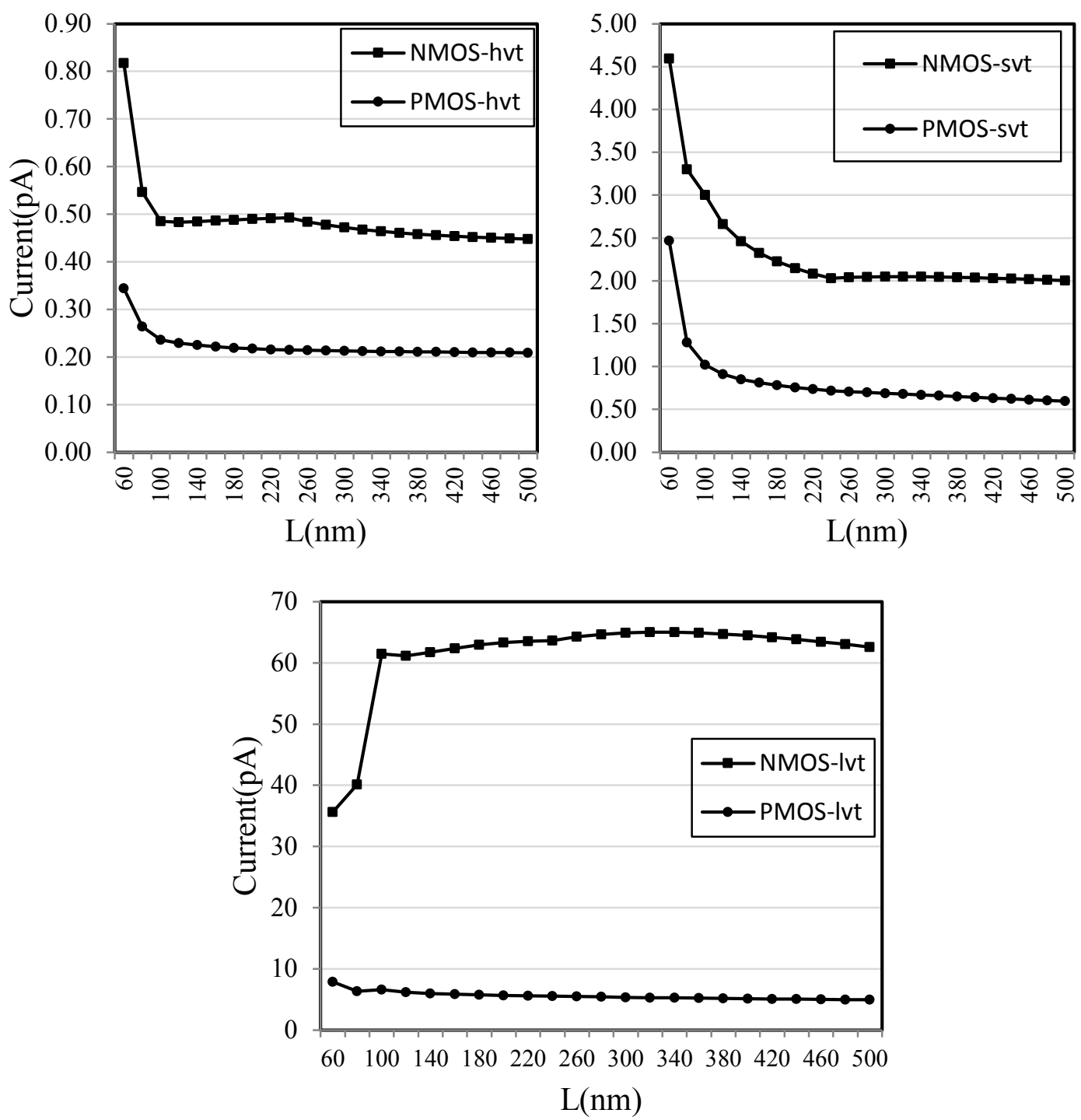

Figure 18 Ioff versus $L\left(W=W_{\min }=120 \mathrm{~nm}, V_{d d}=200 \mathrm{mV}, 65 \mathrm{~nm}\right.$ process $)$.

As shown in Table 4 for an hvt-NMOS transistor, at a larger value of $L$ (of $120 \mathrm{~nm}$ versus the minimum $60 \mathrm{~nm}$ ) the percentage difference between the current values in the processing corners compared to the typical current value decreases. This is also true for all other NMOS and PMOS transistors. Therefore, longer devices are more predictable and more reliable. 
Table 4 Process variation effect on $I_{o N}\left(L=L_{\min }=60 \mathrm{~nm}, W=W_{\min }=120 \mathrm{~nm}, V_{d d}=200 \mathrm{mV}, T=27^{\circ} \mathrm{C}\right.$, $65 \mathrm{~nm}$ process).

\begin{tabular}{|l|c|c|}
\hline & $\begin{array}{c}\text { hvt-NMOS-L-60nm } \\
(\mathbf{n A})\end{array}$ & $\begin{array}{l}\text { hvt-NMOS-L-120nm } \\
(\mathbf{p A})\end{array}$ \\
\hline TT & 0.122 & 37.94 \\
\hline FF & 2.28 & 259.3 \\
\hline FS & 0.696 & 170.4 \\
\hline SF & 0.03 & 8.82 \\
\hline SS & 0.013 & 7.14 \\
\hline
\end{tabular}

\subsection{Comparing Charge and Discharge Currents}

In the subthreshold region both NMOS and PMOS transistors can be used for charging and discharging a node, especially that unlike in the superthreshold case $V_{t h}$ drop is not applicable. The test bench for measuring the average charging (or transferring a "1") and discharging (or transferring a "0") currents through an NMOS transistor is shown in Figure 19. A similar test bench is used for the PMOS counterpart.

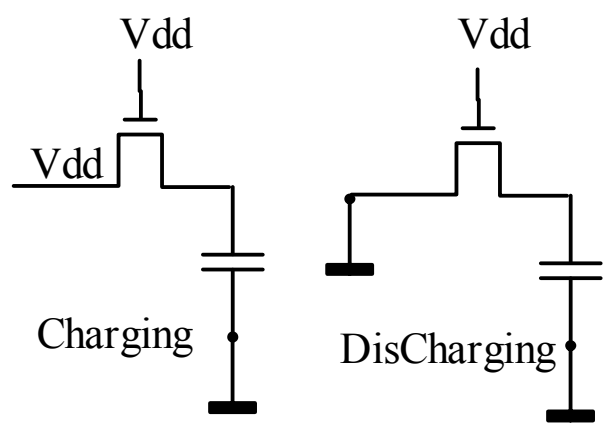

Figure 19 Test benches used for charging and discharging of NMOS.

Table 5 lists the measured charging and discharging current values, for the hvt-type NMOS and PMOS transistors, through schematic simulations. Note that the average charging (discharging) current of the PMOS (NMOS) transistor is almost 100 times larger than its average discharging (charging) current. Also, the average charging (discharging) current 
of the PMOS (NMOS) transistor is almost 100 times larger than the NMOS (PMOS) transistor's average charging (discharging) current. Hence, although both NMOS and PMOS transistors can be used for either charging or discharging purpose to avoid the use of full transmission gates and save energy, the circuit would be much slower.

Table 5 Average currents hvt- NMOS and hvt-PMOS $\left(L=L_{\min }=60 \mathrm{~nm}, W=W_{\min }=120 \mathrm{~nm}, V_{d d}=200 \mathrm{mV}, 65 \mathrm{~nm}\right.$ process $)$.

\begin{tabular}{|c|c|c|}
\hline & Icharging $\mathbf{( p A )}$ & Idischarging (pA) \\
\hline NMOS & 1.11 & 102.6 \\
\hline PMOS & 80.09 & 1.093 \\
\hline
\end{tabular}

\subsection{Improving Robustness of Subthreshold Circuits by Biasing the Body}

Some investigation and design work was initiated to improve the robustness of subthreshold CMOS circuits by manipulating the body-bias of transistors. However, this work was not pursued, because it requires a triple-well CMOS process that is not currently available to us. Hence, the work is moved to Appendix A for the record.

\subsection{Summary}

In this chapter, we presented the effects of transistor sizing on the threshold voltage and (ON and OFF) currents of N-channel and P-channel MOSFETs in the low-, standard-, and high-threshold voltage cases.

In the following chapter, the small building blocks that constitute latches and flipflops are presented along with the use of multi-threshold MOSFETs to design and enhance the reliability of subthreshold flip-flops. Multi-threshold devices of minimum size will be used to design the flip-flops in the $65 \mathrm{~nm}$ technology on the bases that using larger transistors generally degrades the power dissipation. In later chapters we will show that this assumption is not always true, and will incorporate the transistor sizing technique. 


\section{Chapter 4. Proposed Design and Testing Ap- proach}

The power consumption of circuits is critically significant in modern VLSI circuits, especially for low-power applications. At different levels of digital design, power optimization techniques are applied. Designing low-power logic is one of the most significant approaches in attaining this aim. Among most widely logic blocks are flip flops (and latches that constitute them). Therefore, they play a very important role in setting the performance of digital ICs [41], [42]. When estimating the power dissipation of a system, flip-flops are a main power consumption factor [39].

The main performance parameter of a flip-flop is $T_{c-q}$, i.e. delay from the active edge of the active clock edge to the corresponding switching in the output. This parameter in turn, is related to the load capacitance of the clock $\left(C_{c l k}\right)$ that is basically the gate capacitances of the flip-flop transistors driven by the clock. The power consumed by the clock tree is also directly proportional to $C_{c l k}$ [43]. Therefore, in ultra-low-power circuits the aim is to use the smallest possible transistors in the flip flops, although this usually means sacrificing the performance. Using smaller transistors also reduces the leakage current and, hence, the static power dissipation of the circuit.

In this chapter two basic flip-flop topologies are presented for operation in the subthreshold region. Also, measurement test benches for static and dynamic power dissipations, maximum frequency of operation, and timing restrictions are proposed.

\subsection{Latch}

A D latch (or Delay latch) is a logic device that can hold the value of a single input bit [11]. A standard transparent-high (transparent-low) D latch is transparent when its clock input is high (low), which means a change in the input $D$ will be seen in the outputs $Q$ and $Q_{b}$ immediately after the logic propagation delay time. When the clock is low (high), a transparent-high (transparent-low) D latch is said to be opaque and changes in $D$ does not replicate in $Q$. 


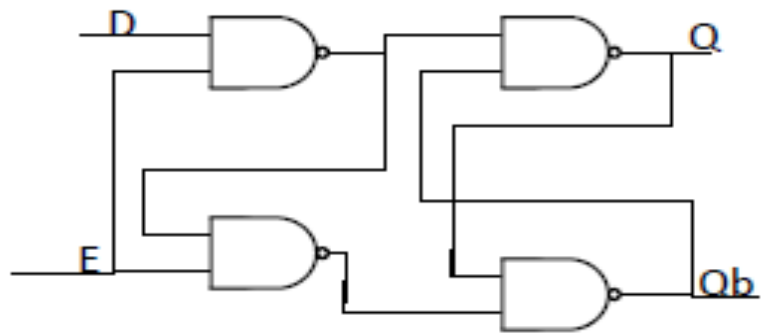

Figure 20 Logic Diagram for a NAND-based (transparent-high) D latch.

The (transparent-high) D latch circuit can be built by four NAND gates as shown in Figure 20. The NAND gates are enabled by signal $E$ that is usually the clock. It is also possible to build a $\mathrm{D}$ latch with fewer transistors by using a combination of regular inverters, tri-state inverters, and transmission gates (TG). D latches built in this way have lower delay and power consumption. Figure 21 shows an inverter latch that consists of two crosscoupled inverters and is considered the heart of a D latch [11]. The inverters act as a feedback loop to provide the latching mechanism.

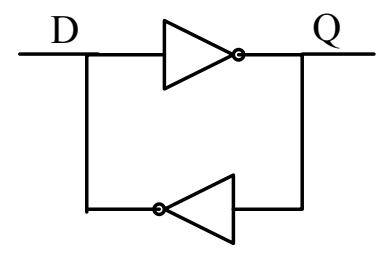

Figure 21 Logic Diagram for an inverter latch.

Figure 22 shows a (transparent-high) D latch based on TG. Here the feedback is open when the clock is high and the data value is passed to the output. The feedback is closed and the data is stored when the clock is low [11]. The inverter and the TG in the feedback act like a tri-state inverter and, hence, can be simply replaced by one. 


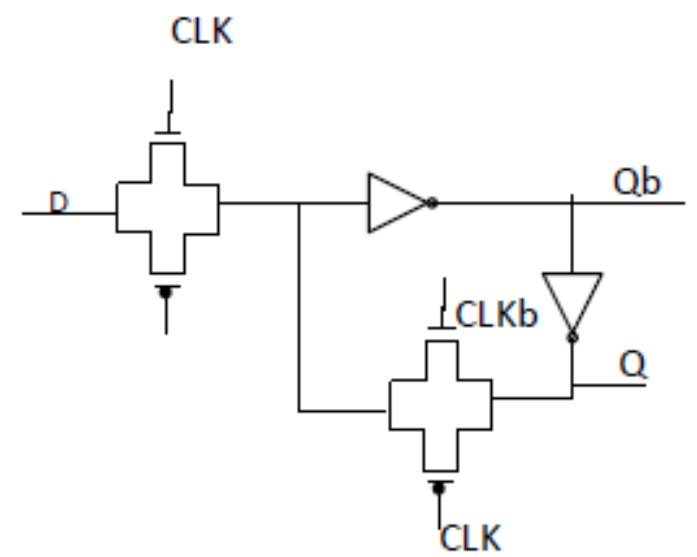

Figure 22 Diagram for a (transparent-high) D latch based on Transmission Gates (TGs).

\subsection{Flip-Flop}

A D flip-flop, also known as an edge-triggered flip-flop or simply a flip-flop, holds the value of a single bit like a latch, but it is never (ideally) transparent. In a positive-edgetriggered (negative-edge-triggered) flip-flop, the stored value is updated when a rising (falling) edge event occurs at the clock input. The symbol for a positive-edge-triggered flip-flop is shown in Figure 23 [19].

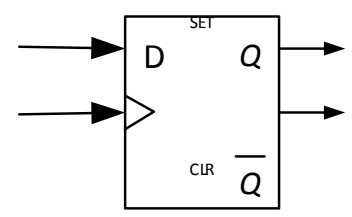

Figure 23 The (positive-edge-triggered) flip-flop symbol.

A basic D flip-flop is constructed by using two D-latches with opposite clock phase inputs, as shown in Figure 24. In this positive-edge-triggered case, the first latch is transparent low and detects changes in the data signal when the clock is low, and propagates the signal to the input of the second latch. When the clock signal is rising, the second latch, which is a transparent-high type, propagates this input value to the flip-flop output $Q$. If the clock phases are non-overlapping, the flip-flop will not be ever transparent, which is a requirement for proper functionality. In a negative-edge-triggered flip-flop the positions of 
the two latch types are switched. Since most commonly used flip-flops are positive-edgetriggered, hereafter the term flip-flop refers to this type.

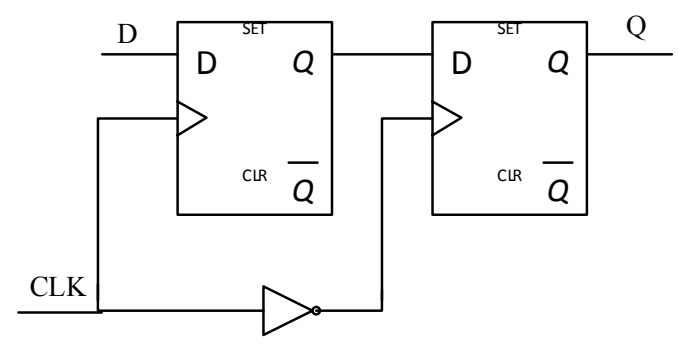

Figure 24 A positive-edge-triggered D flip-flop.

\subsection{Flip-Flop Structure}

The flip-flop can be implemented in different ways. This section describes the two flipflop circuits studied in this thesis. Although there are other flip-flop circuits, some of which may have fewer transistors, they are not as robust against PVT due to sever timing restrictions and, hence, not as appealing for subthreshold operation [22]. Nevertheless, a separate research project in the future may suggest ways to make them compatible with subthreshold operation.

\subsubsection{Transmission Gate Flip-Flop (TG flip-flop)}

The flip-flop can be constructed using CMOS transmission gates as shown in Figure 25. The first stage, which is a transparent-low latch, is driven by the clock signal, while the second stage, which is transparent-high latch, is driven by the inverted clock signal.

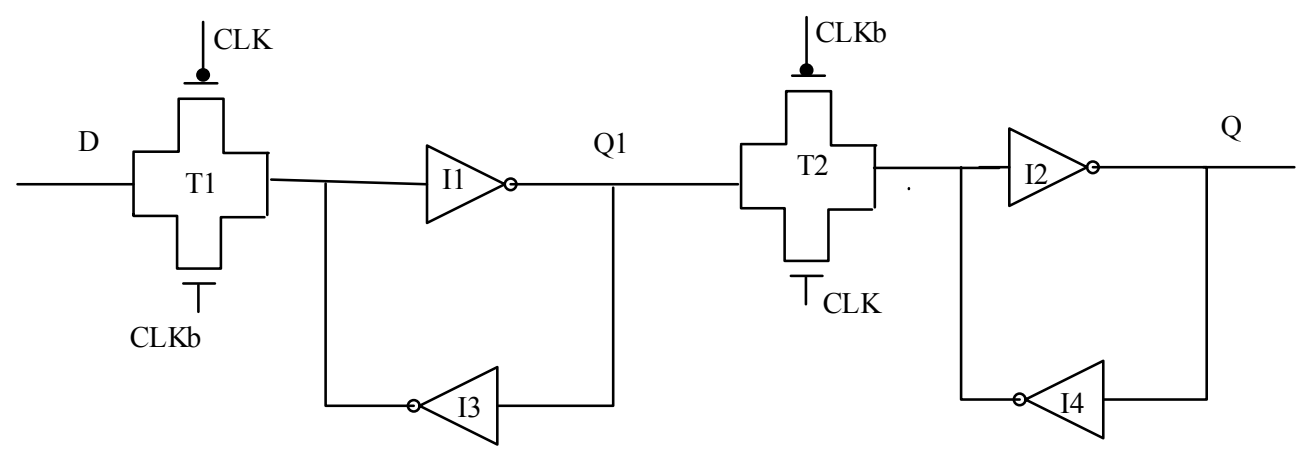

Figure 25 Transmission Gate Flip-Flop circuit. 
When the clock is low, the first stage samples the input $D$ while the second stage holds the previous stored value. When the clock changes from a logic " 0 " to logic " 1 ," the first latch stops sampling the input and stores the $D$ value at the time of the clock transition. At the same time, the second latch becomes transparent, passing the stored first latch value $Q_{I}$ to the output of the second stage $Q$. The input cannot affect the output anymore because the first stage is disconnected from the input $D$. When the clock changes again from a logic " 1 " to " 0 ," the second latch locks in the first latch output and the first stage starts sampling the input again. This circuit is also known as the single-threshold transmission gate flipflop. The TG flip-flop is perhaps the most widely used and one of the fastest and lowestpower-consuming flip-flop circuits [19], [44].

\subsubsection{Single Phase Flip-Flop (SP flip-flop)}

Generally, both the clock signal and its inversion are needed for operating a flip-flop. Because, as in the TG flip-flop, a flip-flop is implemented by connecting two complementary $\mathrm{D}$ latches in series. The inverted clock can be supplied globally together with the clock or generated locally using an inverter. However, globally supplying the clock and its inversion may result in them being skewed in relation to each other as the two signals travel across the chip and may be heavily loaded. The skew problem also exists with the locally generated inverted clock as well, but it might be less challenging to reduce. Therefore, a flipflop with a single-phase clocking scheme is preferred [45], if the cost in performance degradation can be tolerated.

A single-phase flip-flop circuit is shown in Figure 26. It is constructed using MOS single-phase pass gates instead of CMOS TGs. The first stage is driven by a low clock signal through a PMOS pass gate, while the second stage is driven by a high clock signal through an NMOS pass gate. Obviously the feedback pass gate in a stage must be of the opposite type to its forward-path pass gate. 


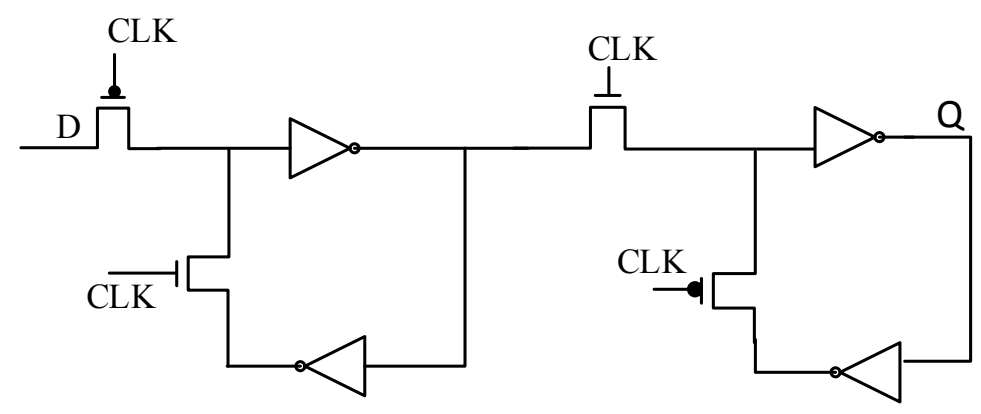

Figure 26 Single Phase Flip-Flop Design.

\subsection{Multi-Threshold MOSFETs}

A multi-threshold voltage CMOS (MT-CMOS) technology provides for fundamentally faster and less-leaky devices that are engineered through the process to provide the desired performance characteristics. Such a technology enables the manufacturing of transistors with different threshold voltages $\left(V_{t h}\right)$ on a single chip. A transistor with a lower $V_{t h}$ offers a higher switching speed but a penalty is given in the form of higher leakage current. Therefore, lower $V_{\text {th }}$ transistors or cells are used in critical delay paths of the design when a higher speed is demanded [46], [47]. On the contrary, higher threshold voltage results in less leakage current, so lower static power consumption, at the cost of larger delay. By using multiple-threshold voltage devices in a circuit, designers can suppress the leakage current while also meeting certain performance requirements.

\subsection{Test Benches}

Before proceeding with the flip-flop circuit implementations, it is essential to design appropriate test benches for testing the functionality and measuring the metrics. After ensuring that a flip-flop circuit implementation is functional, the following metrics should be evaluated. 
1. Static power consumption

2. Dynamic power consumption

3. Maximum operation frequency

4. Timing restrictions

5. Reliability

In the main test bench, the output $Q$ is connected to the input $D$ through five inverters for automatic oscillation, as shown in Figure 27. We could have used three inverters, but the feedback could become too fast. If the flip-flop is functional, the output switches at every positive edge of the clock. Two inverters are used to generate the clock signal and its inversion. All flip-flop transistors are initially of minimum size, but the $Q$-to- $D$ inverters and the clock-generation inverters are made of wider transistors to produce a sharp input $D$ and sharp clock signals. The flip-flop is simulated with a $V_{d d}$ of $200 \mathrm{mV}$.

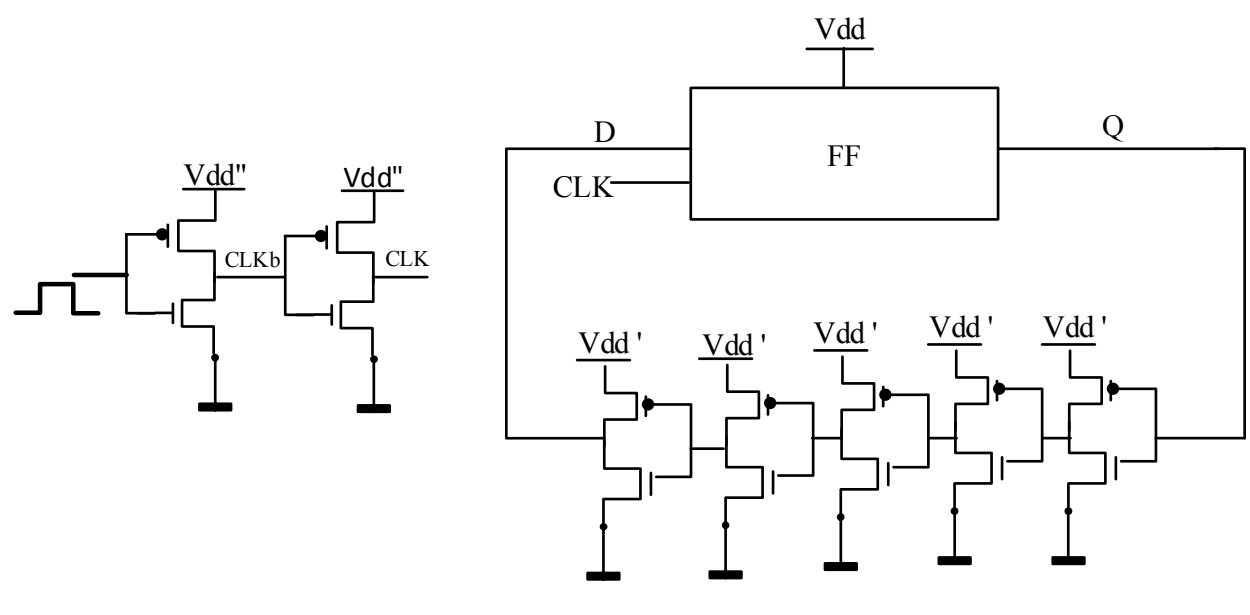

Figure 27 Flip-flop's main test bench.

\subsubsection{Static Power Measurement}

The static power consumption is measured by having steady logic values at the input, output, and internal nodes of the flip-flop. The clock signal should be disabled and the five $Q$ to- $D$ inverters shall be removed. As shown in Figure 28, there are eight possible cases of clock, $D$, and $Q$ values when the flip-flop is idle. The average static power consumption over the eight cases will be reported. Note that in all eight cases it is important to set all internal nodes of the flip-flop to an appropriate logic value based on $D$ and $Q$. When the 
clock is low, all internal node logic levels are automatically set by $D$ and $Q$. However, when the clock is high, $Q_{0}$ has to be manually initialized.
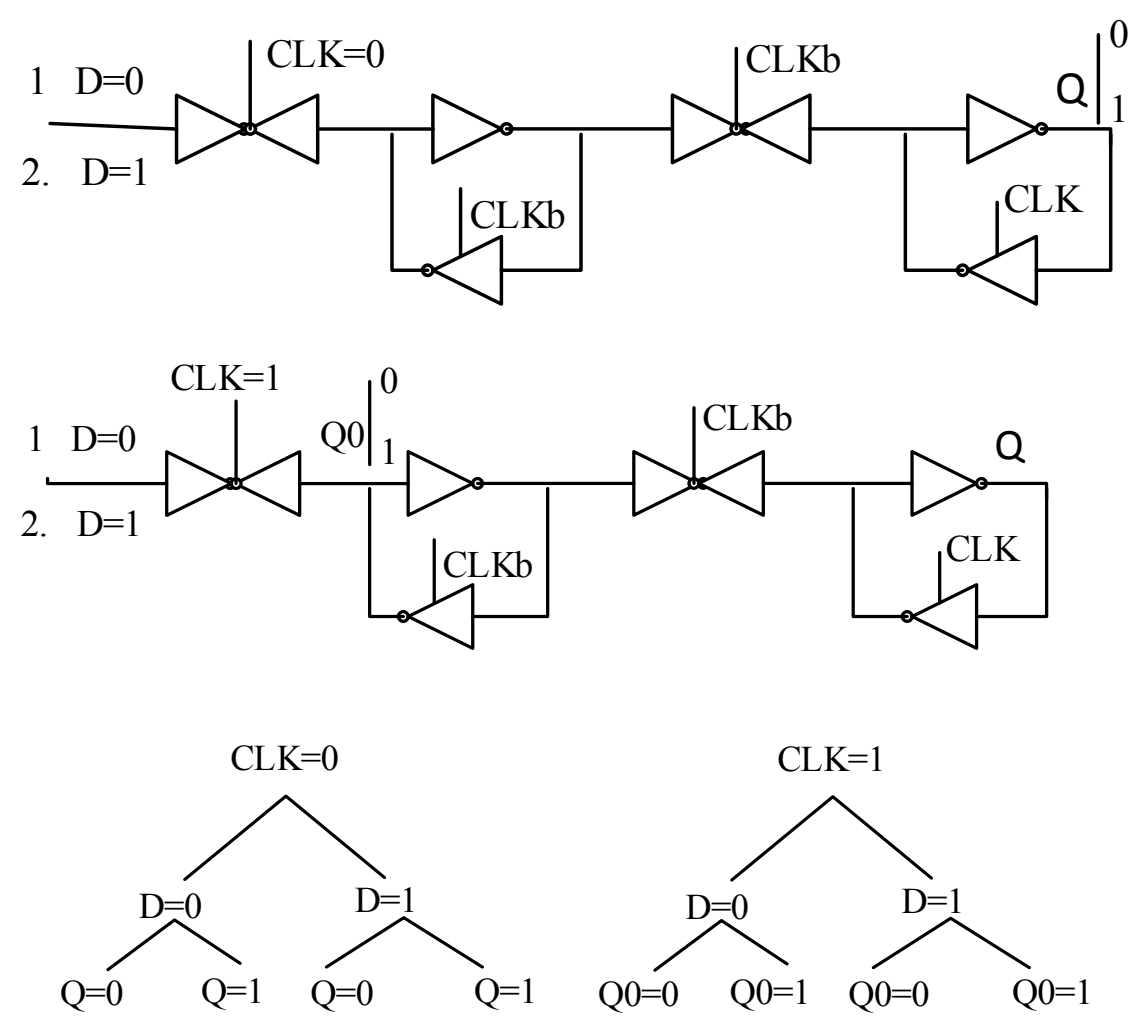

Figure 28 Static power test bench.

\subsubsection{Dynamic Power Consumption Measurement}

The test bench of Figure 27 is used to measure the total dynamic power consumption of the flip-flop. If the power consumption of the clock-generation inverters is $P_{c l k i n v}$, the power consumption of the five $Q$-to- $D$ inverters is $P_{i n v Q-D}$, and the power consumption of the whole test bench is $P_{\text {total }}$, then the total power consumption of the flip-flop is given by

$$
P_{F F}=P_{\text {total }}-\left(P_{\text {clkinv }}+P_{\text {invQ-D }}\right)
$$

and the dynamic power consumption of the flip-flop alone, $P_{d F F}$, can be obtained from 


$$
P_{F F}=P_{d F F}+P_{s t F F}
$$

where $P_{s t F F}$ is the static power consumption of the flip-flop only.

\subsubsection{Maximum Operation Frequency Measurement}

The flip-flop would be operating at its maximum frequency if a new data is sampled as soon as the old data appears at the output. Therefore, the clock period cannot be shorter than the delay between $D$ and $Q$ when the transmission gates in between are enabled. The test bench for measuring the maximum operation frequency based on this critical path is depicted in Figure 29.

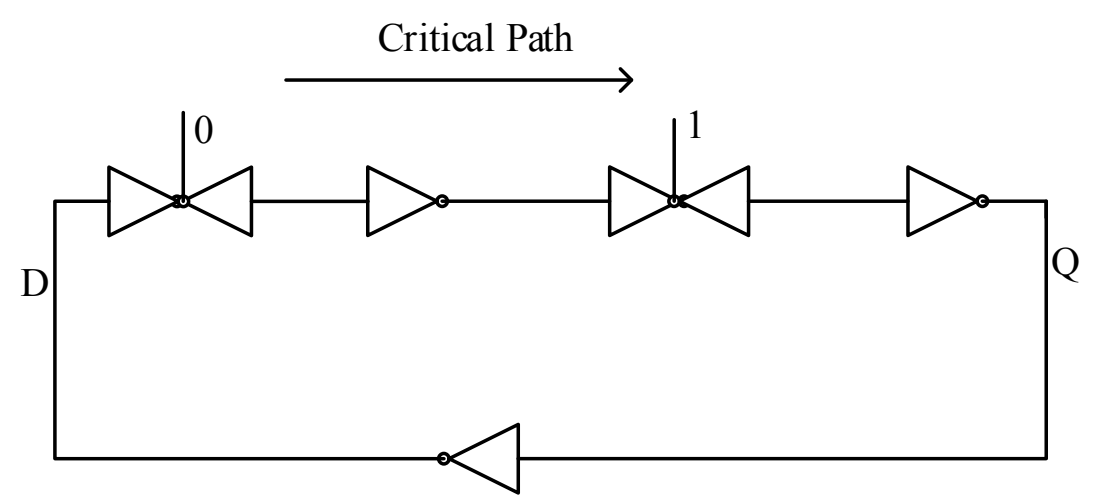

Figure 29 Test bench maximum operation frequency measurement.

\subsubsection{Setup Time Test Bench}

The setup time $\left(T_{\text {setup }}\right)$ of the flip-flop is the minimum period in which the input must be stable before the active edge of the clock. This is the delay between $D$ and $Q_{1}$ in Figure 30, where the setup-time test benches for the TG flip-flop are shown. 

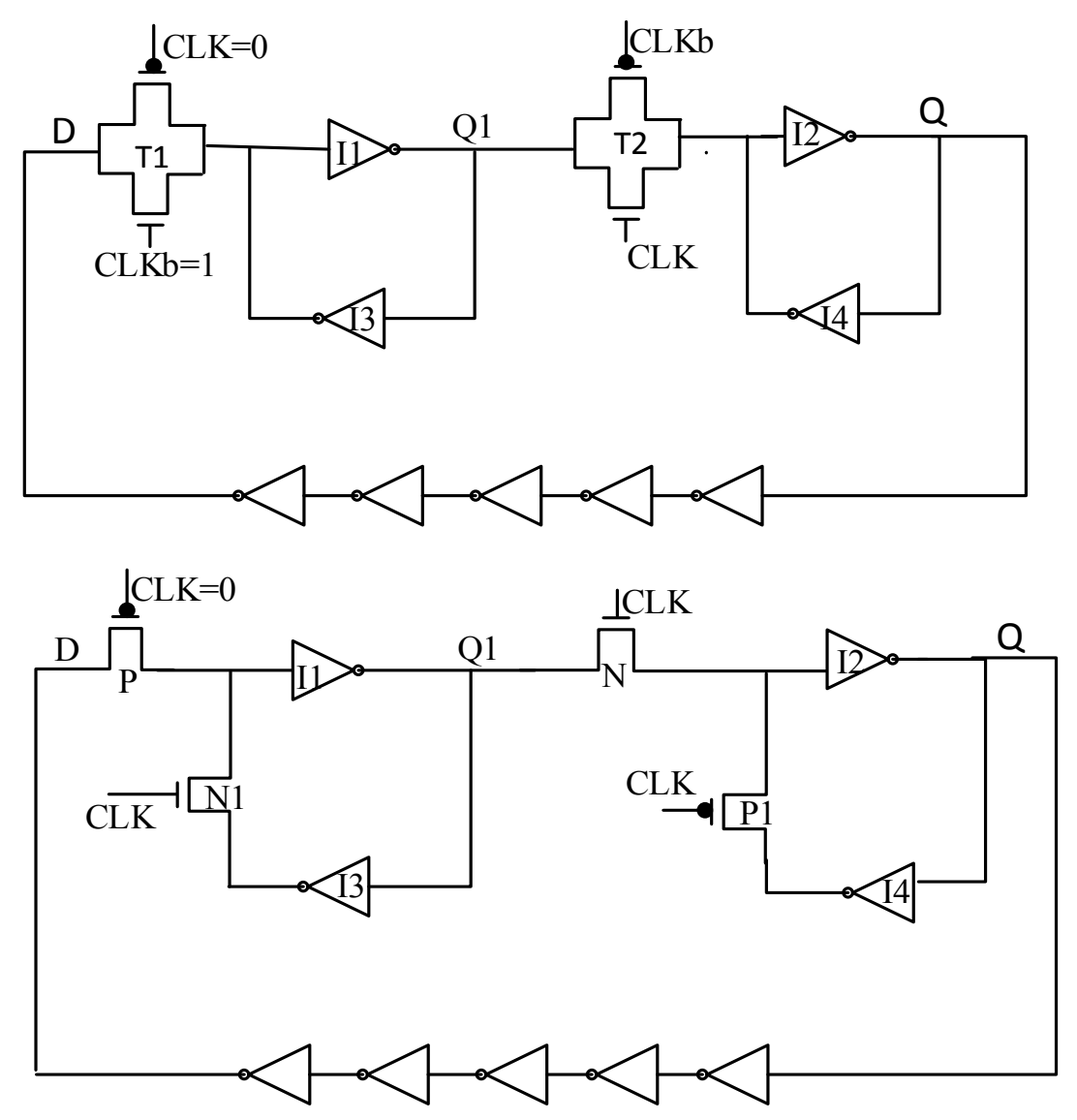

Figure 30 The setup-time test bench of TG flip-flop.

Accordingly, the setup time of the TG flip-flop is

$$
T_{\text {setup-TG }}=T_{T 1}+T_{I 1}
$$

and the setup time of the SF flip-flop is

$$
T_{\text {setup-SP }}=T_{P}+T_{I 1}
$$

\subsection{Reliability Evaluation}

Monte Carlo analysis simulates the effects of process variations by using random statistical process-variation data provided by the technology kit vendor. Multiple simulations are run with random process variations to estimate the yield of the design. For the flip-flop at least 
200 runs is chosen. The most important parameters of Monte Carlo simulations based on [21] are:

1. Mean $(\mu)$ is the average values

2. Standard deviation or sigma $(\sigma)$ is a measure of how spread out numbers are

3. Relative Sigma (б) or relative standard deviation or coefficient of variation shows the extent of variability in relation to mean of the population, it is expressed as

$$
6=\frac{\sigma}{\mu}
$$

4. Minimum and maximum values

\subsection{Summary}

This chapter presented the flip-flop circuits to be implemented. It also introduced our proposed method of measuring the static power, switching power, maximum frequency, setup time, and reliability of the flip-flop. In the next chapter, the detailed implementations of the flip-flop circuits are proposed to maximize the yield and minimize the power consumption. 


\section{Chapter 5. Reliable Subthreshold Flip-Flop De- sign Using Multi-Threshold MOSFET Techniques}

This chapter investigates the flip-flop design in the TSMC $65 \mathrm{~nm}$ LP CMOS. It describes the design procedure and comparisons of different flip-flops configurations and their building blocks. Flip-flops can be designed as a static or dynamic component. Static flip-flop designs require some feedback to retain their output value while the power supply is $\mathrm{ON}$. Dynamic flip-flops do not require feedback and generally maintain their output as a charge on capacitors. The storage capacitances in a dynamic flip-flop must be periodically refreshed; otherwise, the charge on these nodes will leak away, resulting in invalid data [48]. Therefore, dynamic designs are more sensitive to leakage currents than static designs. Due to decreased $I_{O N} / I_{O F F}$ ratio in subthreshold operation, dynamic circuits are more sensitive to process variations. Only static flip-flops have been considered for this work. Based on Section 4.3, two different flip-flops are considered as follows.

- TG Flip-Flop

- $\quad$ SP Flip-Flop

As the size and complexity of chip designs are rapidly growing, reliability is becoming an important factor to consider when designing nanometer circuits and systems. In this chapter, we will present the techniques involved in designing high-performance and ultralow-power flip-flops, while focusing on reliability by using multi-threshold MOSFETs.

\subsection{Building Blocks}

The major flip-flop building blocks include the inverter, transmission gate and tri-state inverter. 


\subsubsection{Inverter}

The inverter takes an input signal and inverts it to the output. As Figure 31 shows, it consists of one NMOS (pull-down) and one PMOS (pull-up) transistor.

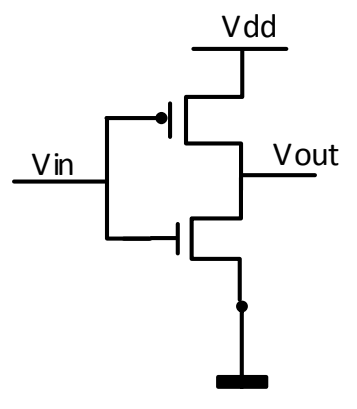

Figure 31 CMOS Inverter.

\subsubsection{Transmission Gate (TG)}

The transmission gate includes one NMOS and one PMOS transistor linked in parallel as shown in Figure 32. The transmission gate acts as a switch, where the $C L K$ signal is either high or low [48]. When CLK is high, both NMOS and PMOS conduct, and while CLK is low, both NMOS and PMOS are " 0 ". The parallel connection causes the transmission gate to conduct the full voltage range from " 0 " to $V d d$.

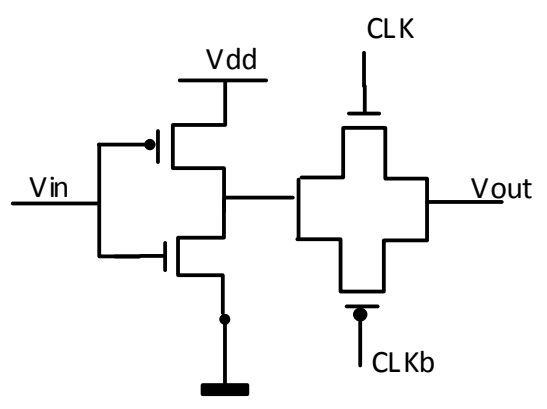

Figure 32 Transmission Gate. 


\subsubsection{Tri-State Inverter (TSI)}

A clocked inverter operates similarly to a conventional inverter, except that it is controlled by an enabling signal. The schematic of the clocked inverter can be seen in Figure 33, where signals $C L K$ and $C L K b$ are the set signals. These are normally connected to the clock signal, so that the inverter inverts while the clock is either low or high. The control transistors are placed between the input-signal transistors as data is arrived after clock. Consequently, the unwanted effects of charge sharing, which decrease the output swing and can cause instability [49], are removed.

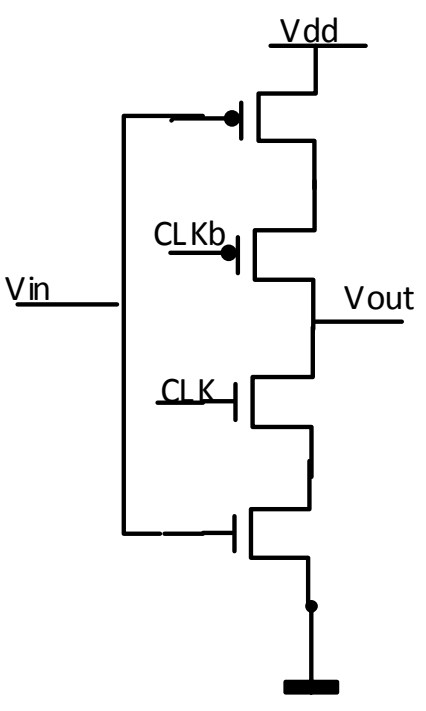

Figure 33 Tri-state inverter.

\subsubsection{Inverter, Tri-State, and Transmission Gate Inverter Simulation Results}

In all three inverter test benches, high threshold voltage transistors are uses to decrease leakage current. All simulations are done using a supply voltage of $200 \mathrm{mV}$ to make sure the transistors are in weak inversion.

The worst case delay and power for the different inverters are measured and presented in Table 6. The regular inverter has the smallest delay and power consumption. Furthermore, Tri-State inverter delay is much lower than Transmission gate. Hence, TSI is superior over transmission gate for flip-flop building block. 
Table $6 T_{c-q}$ and $P_{t}$ of inverter, TSI, and TG $\left(L=L_{\min }=60 \mathrm{~nm}, W=W_{\min }=120 \mathrm{~nm}, V_{d d}=200 \mathrm{mV}\right.$, $T=27^{\circ} \mathrm{C}$, hvt, $65 \mathrm{~nm}$ process).

\begin{tabular}{|l|c|c|}
\hline & Delay $(\boldsymbol{\mu s})$ & Power $(\mathbf{p W})$ \\
\hline Inverter & 1.89 & 0.6 \\
\hline Tri-State Inverter & 3.2 & 1.01 \\
\hline Transmission Gate & 11.34 & 0.98 \\
\hline
\end{tabular}

The Monte Carlo simulation results of TSI and TG are shown in Figure 34 for room temperature $\pm 50^{\circ} \mathrm{C}\left(-23^{\circ} \mathrm{C} .27^{\circ} \mathrm{C}, 77^{\circ} \mathrm{C}\right)$. The delay mean and sigma of TSI and TG are presented in Table 7.

Table 7 Mean and sigma of TSI and TG $\left(L=L_{\min }=60 \mathrm{~nm}, W=W_{\min }=120 \mathrm{~nm}\right.$, $V_{d d}=200 \mathrm{mV}, T=27^{\circ} \mathrm{C}$, hvt, $65 \mathrm{~nm}$ process).

\begin{tabular}{|c|c|c|c|c|}
\hline & & $T=-23{ }^{\circ} \mathrm{C}$ & $T=27{ }^{\circ} \mathrm{C}$ & $T=77^{\circ} \mathrm{C}$ \\
\hline \multirow[t]{2}{*}{ Mean $(\mu \mathrm{s})$} & Tri-State Inverter & 8.87 & 3.46 & 2.2 \\
\hline & Transmission Gate & 17.73 & 11.39 & 9.08 \\
\hline \multirow[t]{2}{*}{ Sigma $(\mu \mathrm{s})$} & Tri-State Inverter & 6.7 & 0.52 & 0.04 \\
\hline & Transmission Gate & 14.57 & 1.9 & 0.3 \\
\hline
\end{tabular}

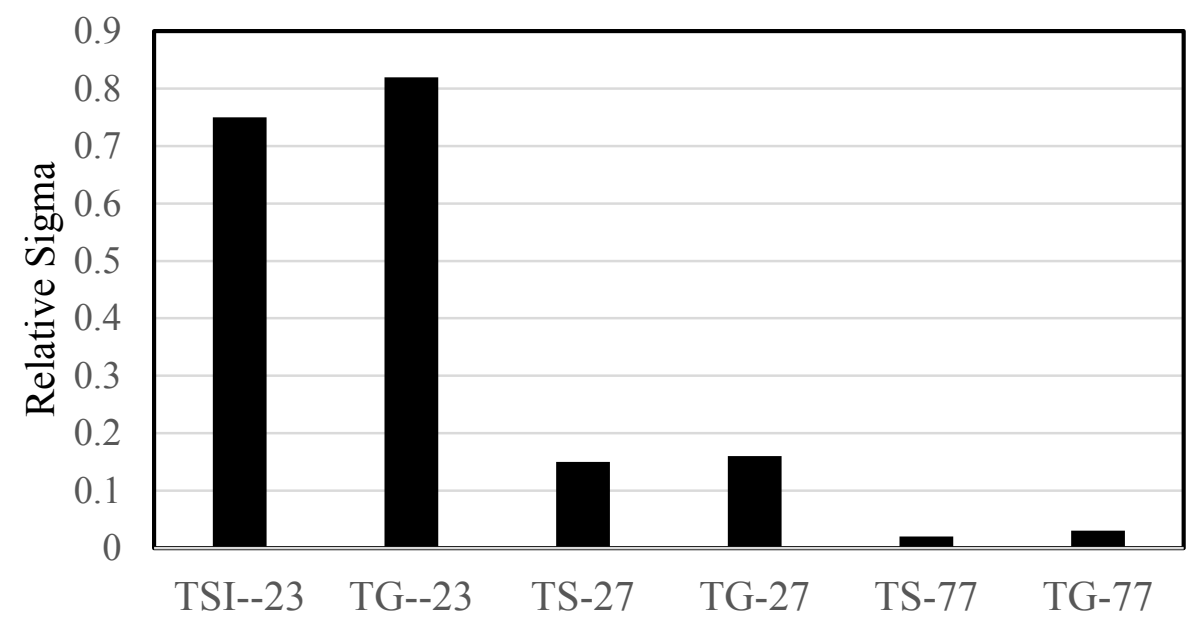

Figure 34 Relative sigma of TSI, and TG $\left(L=L_{\min }=60 \mathrm{~nm}, W=W_{\min }=120 \mathrm{~nm}, V_{d d}=200 \mathrm{mV}, T=-\right.$ $23^{\circ} \mathrm{C}, T=27^{\circ} \mathrm{C}, T=77^{\circ} \mathrm{C}, \mathrm{hvt}, 65 \mathrm{~nm}$ process).

From Equation (4-23), the dispersion of values are less if relative sigma is smaller. As Figure 34 and Table 7 show, the relative sigma relating to TSI for all temperatures is 
smaller than relative sigma contributing to TG. Therefore, TSI is more reliable compared to TG. Hence, we will use the tri-state inverter as the feedback inverter (D latch) in the flipflop configuration of the next section.

\subsection{Design of PVT-Tolerant Subthreshold Flip-Flops}

In this section, the yield of the transmission gate and single phase flip-flop based on Monte Carlo simulations would be improved. First, we examine the yield of both flip-flops regard to process variations when all their transistors are minimum size $(L=60 \mathrm{~nm}, W=120 \mathrm{~nm})$. Then we use the current versus $L$ and $W$ in section 3.2 to set up the different threshold devices of the critical transistors in circuits to improve the yield. We test the simulation waveforms of the test bench in Figure 27.

\subsubsection{Transmission Gate flip-flop (TG-FF)}

The schematic of the transmission gate flip-flop is shown Figure 35. This structure is implemented using conventional and tri-state inverters, using up 16 transistors.

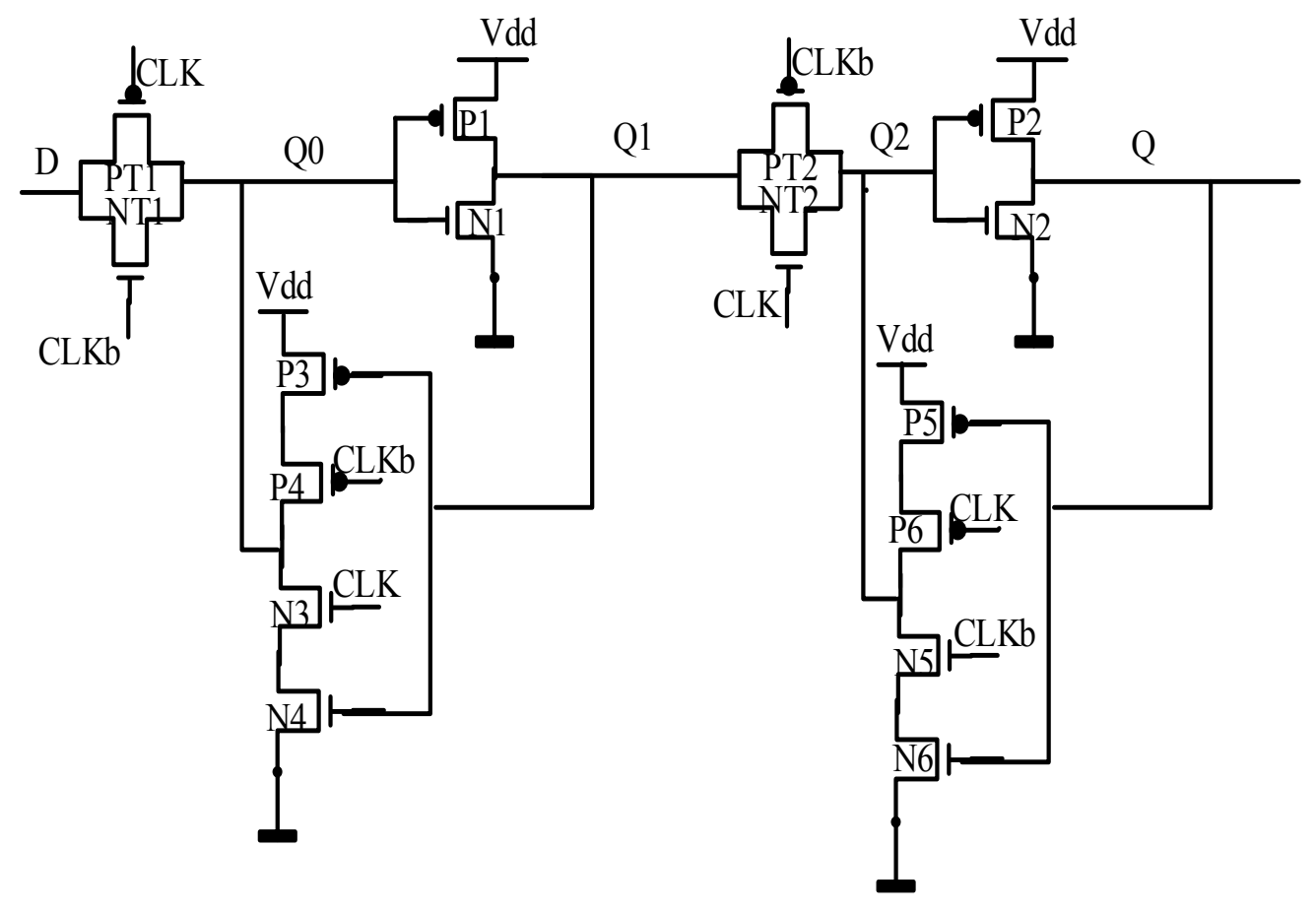

Figure 35 Transmission gate flip-flop schematic. 
An effective method for decreasing leakage currents in subthreshold region employs NMOS and PMOS devices with high threshold voltage and minimum size ( $L n=L p=60 \mathrm{~nm}, W n=W p=120 \mathrm{~nm}$ ). One can utilize specialized circuit topologies to take advantage of the leakage reduction benefits of high $V_{t h}$ transistors. Therefore, high threshold voltage transistors with minimum size are employed in the flip-flop of Figure 35 instead of standard threshold voltage. TG flip-flop is simulated at all corners to extract the transient behaviour. Figure 36 shows the timing diagram of signals CLK, Q in the SS (top waveforms) and SF (bottom waveforms) corners. In the SF corner, flip-flop works correctly. As shown in Figure 36 (a), the flip-flop does not operate properly for the SS process corner when both NMOS and PMOS devices are slow. Since transmission gate transistors (PT1, NT1, PT2, and NT2) have an important role to pass data, they should be faster. According to Figure 15 and Figure 16, the standard threshold voltage transistor's current is almost 10 times the transistor's current of the high threshold voltage one. Hence, multi-threshold technique is required to make faster PT1 and PT2. 


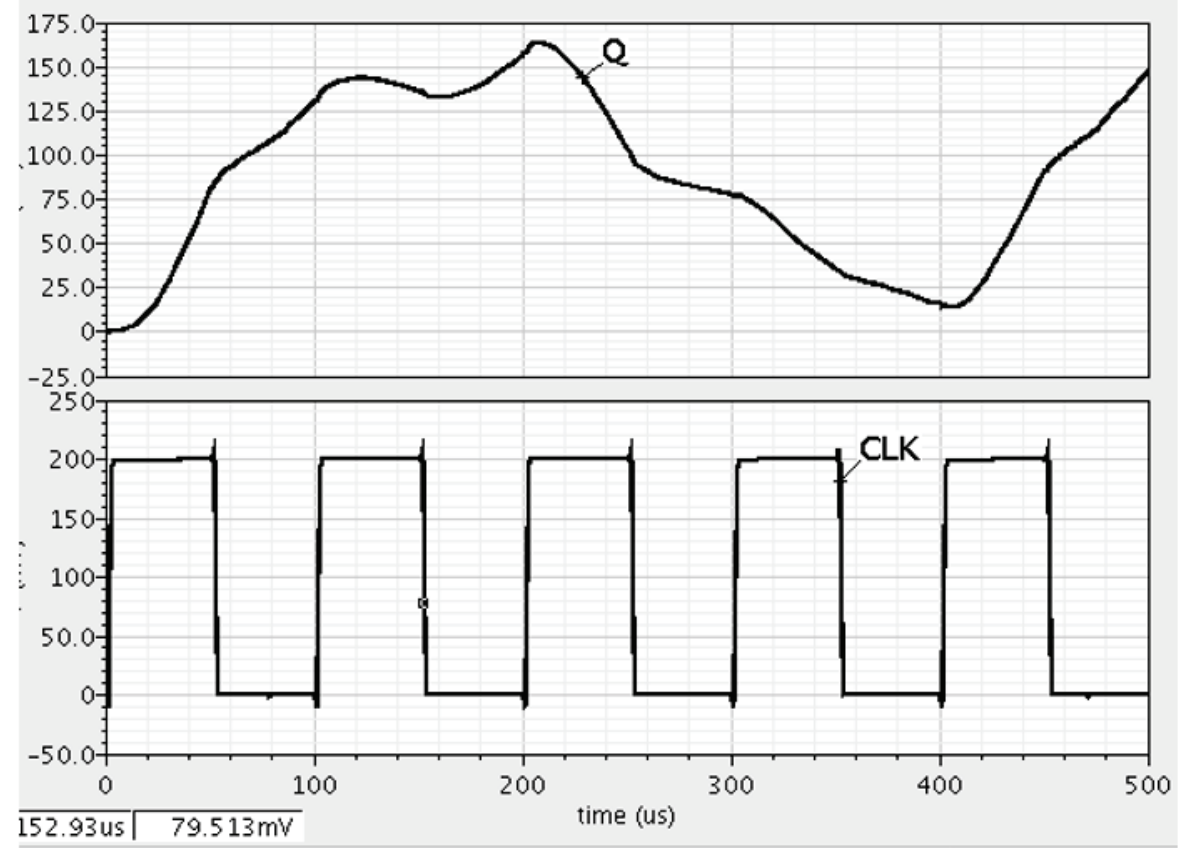

(a)

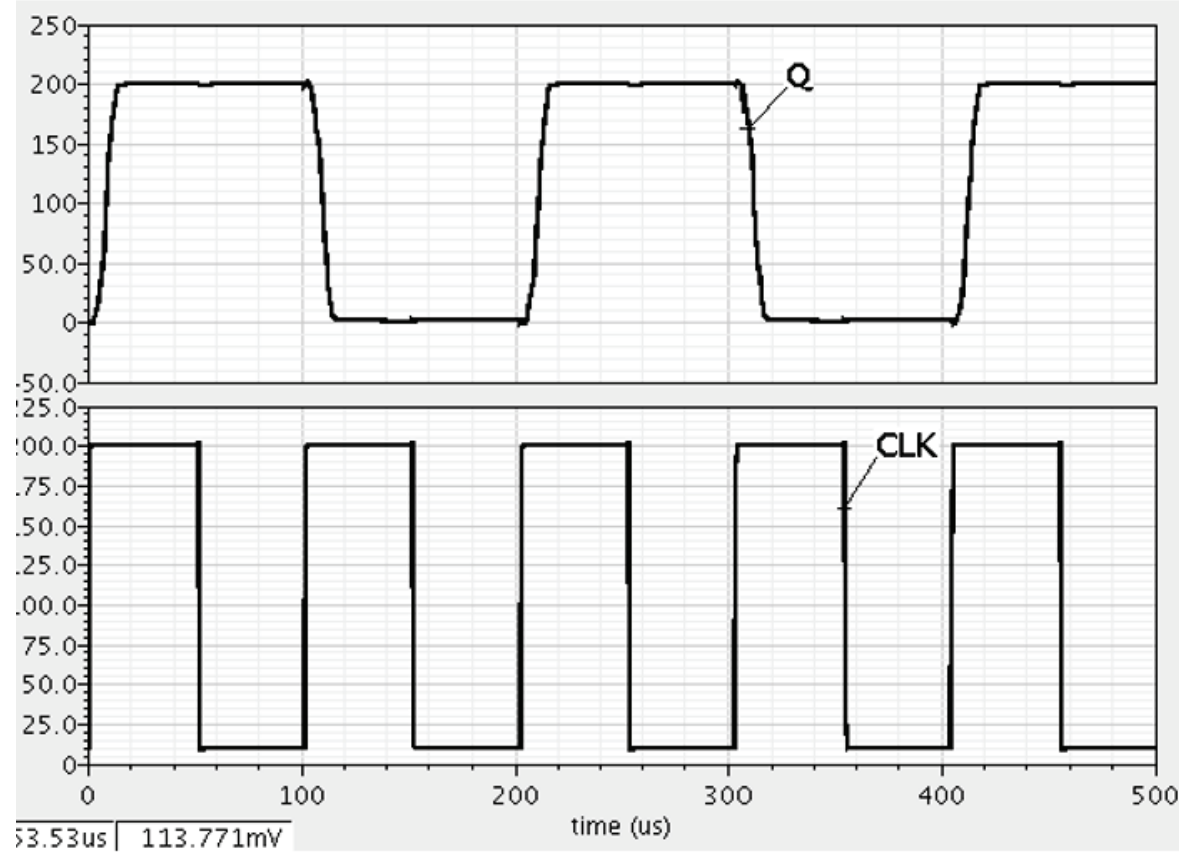

(b)

Figure 36 Transient response of minimum size TG flip-flop at (a) SS corner, (b) SF corner ( $V_{d d}=200$ $\mathrm{mV}, 65 \mathrm{~nm}$ process, hvt transistors).

The flip-flop are considered on three different cases: input, output, and clock pulses in three cases are counted. Moreover, Monte Carlo simulations are used to determine how many of the pulses have "passed". When a pulse has not passed, it means that the flip-flop 
has not functioned properly due to process variations. Figure 37 shows the highest yield when PT1 and PT2 are standard threshold voltage PMOS transistor. Thus, speeding up PT1 and PT2 using standard threshold voltage devices has resulted in higher reliability.

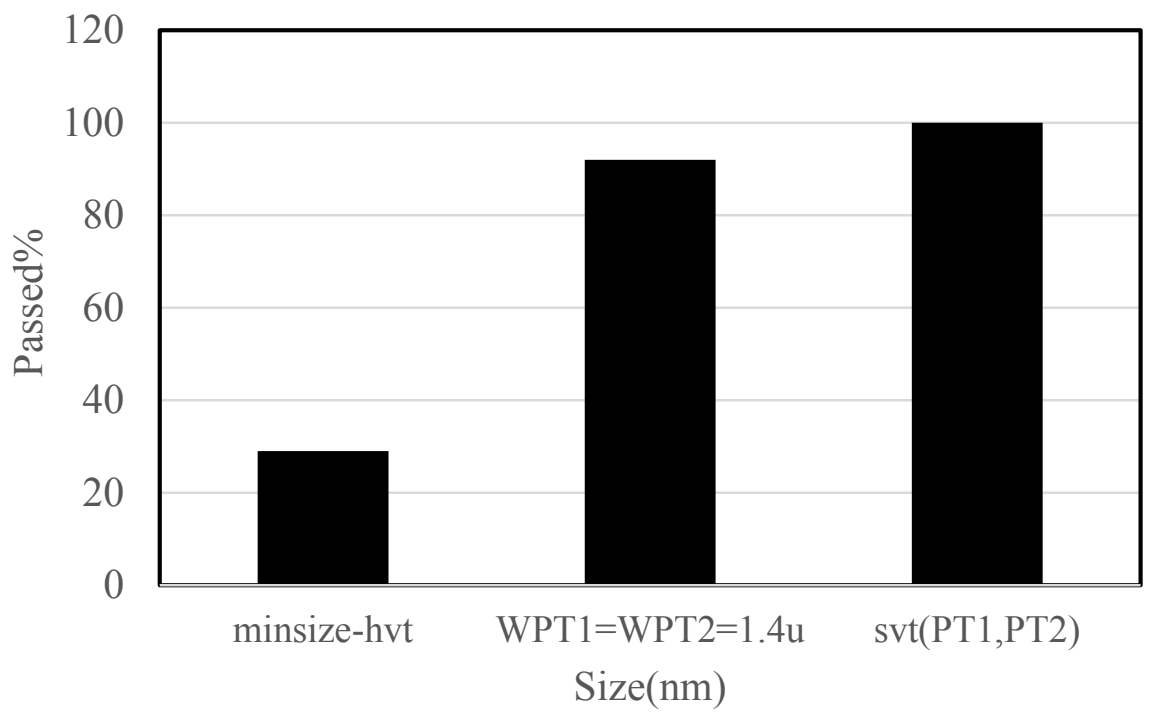

Figure 37 Number of passed of TG flip-flop for different devices ( $V_{d d}=200 \mathrm{mV}, 65 \mathrm{~nm}$ process).

Transient simulations show that using svt for PT1 and PT2 results in rise time and fall time of output is $6.37 \mu$ s and $7 \mu$ s at the output respectively. Since the rise and fall time values are close, a 50\% duty cycle at the output of the flip-flop can be produced. Hence, the flip-flop functions correctly.

\subsubsection{Single Phase flip-flop (SP-FF)}

The single phase flip-flop is shown in Figure 38. This flip-flop is constructed using 2 passgate transistors at the input of the latches, 2 pass-gate transistors in feedback to produce delay. Furthermore, 2 conventional inverters are used in the feedback to hold their state. Hence, there are 12 transistors in this design, which is a less transistor count compared to TG flip-flop. Minimum size high-threshold devices are used for all flip-flop transistors. 


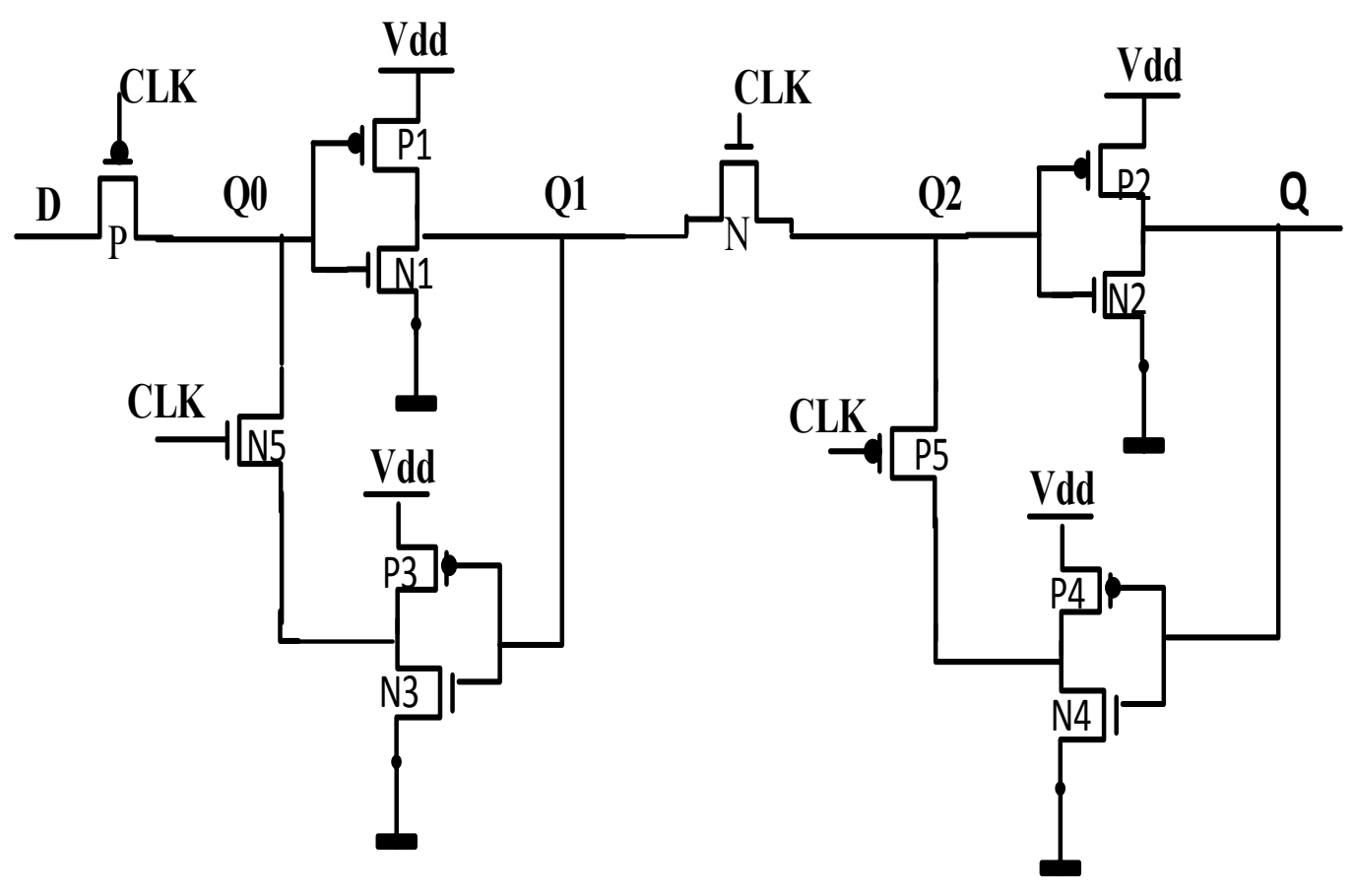

Figure 38 Single phase flip-flop schematic.

The transient simulations of the previous section are repeated for the single-phase flip-flop. These simulations show that the SP flip-flop functions properly for the FF and SF process corners. Thus, it can be said that using minimum size high threshold devices, NMOS transistors are faster than PMOS. Figure 39 shows the output and clock waveforms produced by the SP flip-flop using high-threshold voltage transistors at FS corner (top waveforms) and SF corner (bottom waveforms).

Multi threshold SP flip-flop consists of two paths, namely non-critical and critical. The circuits that hold the state have non-critical paths. The p-channel transistors of critical path should be faster to pass data. Therefore, hvt-transistors $\mathrm{P}, \mathrm{P}_{1}$, and $\mathrm{P}_{2}$ are replaced by standard-threshold voltage devices, resulting in higher yield and reduced leakage.

Using Monte Carlo simulation, the flip-flop yield is measured for three different cases: all transistors are high threshold voltage, all PMOS transistors in the critical path are standard-threshold voltage, and only $\mathrm{P}$ and $\mathrm{P}_{1}$ of the critical path svt devices. As shown in 
Figure 40, when $\mathrm{P}$ and $\mathrm{P}_{1}$ transistors are of standard $V_{t h}$, the highest yield is obtained for the SP flip-flop.

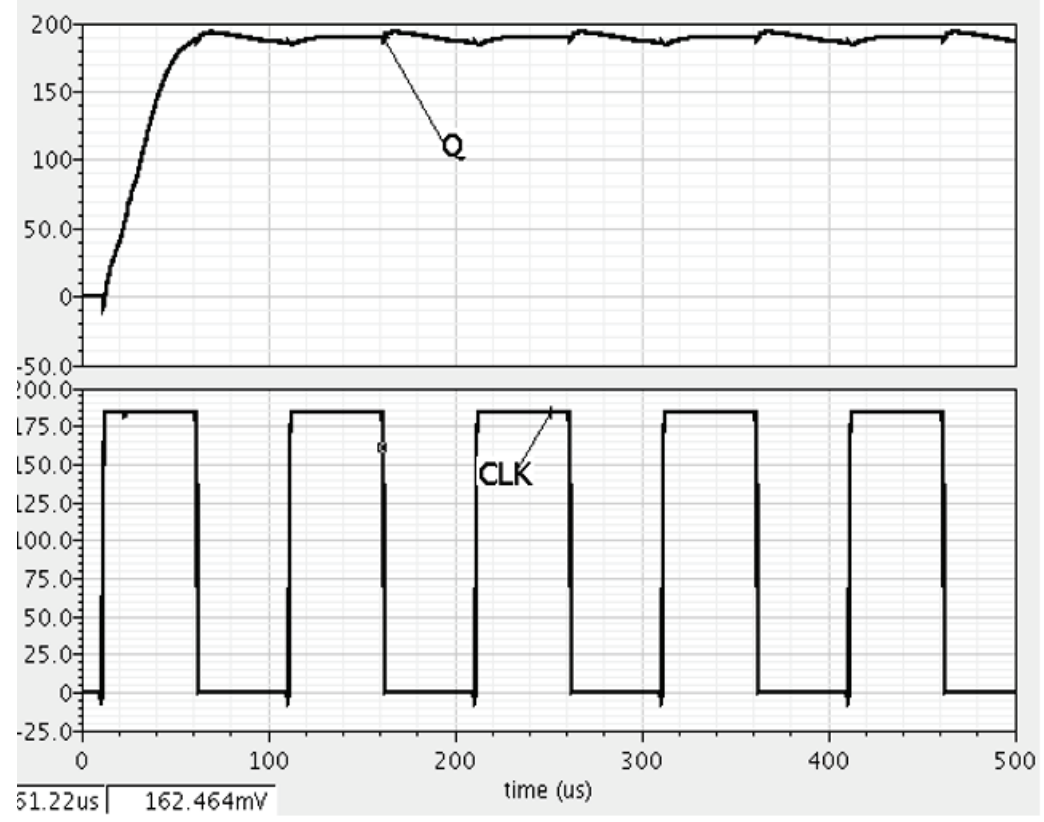

(a)

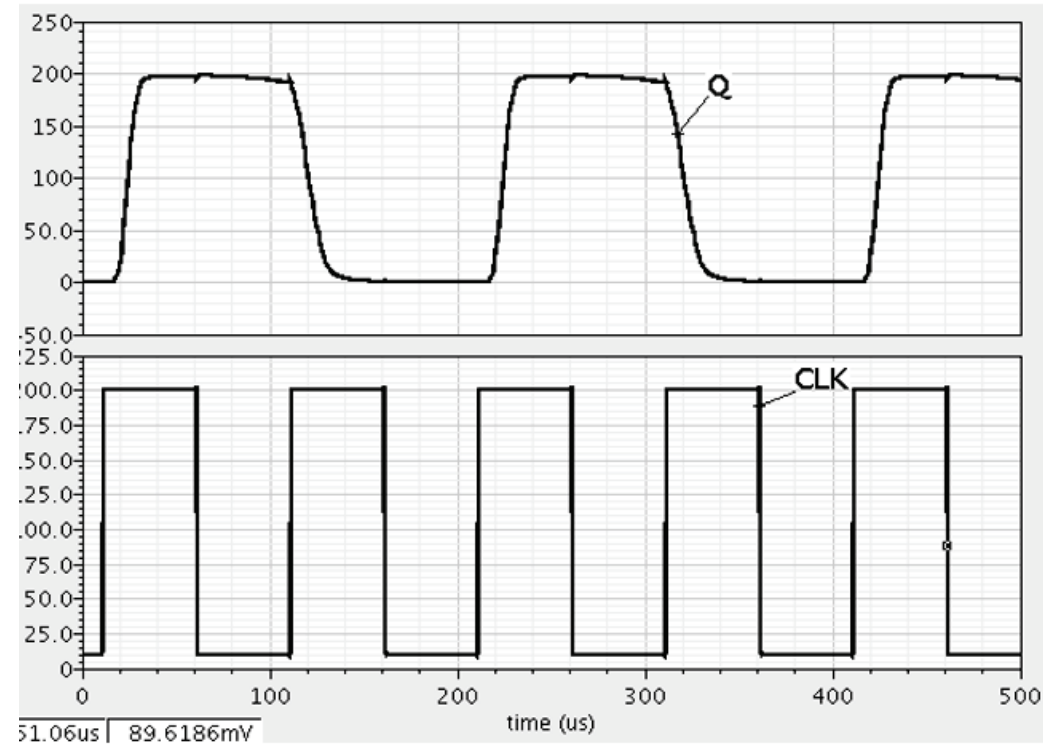

(b)

Figure 39. Transient response of minimum size of SP flip-flop at (a) FS corner (b) $\mathrm{SF}$ corner ( $V_{d d}=200 \mathrm{mV}, 65 \mathrm{~nm}$ process, hvt devices). 


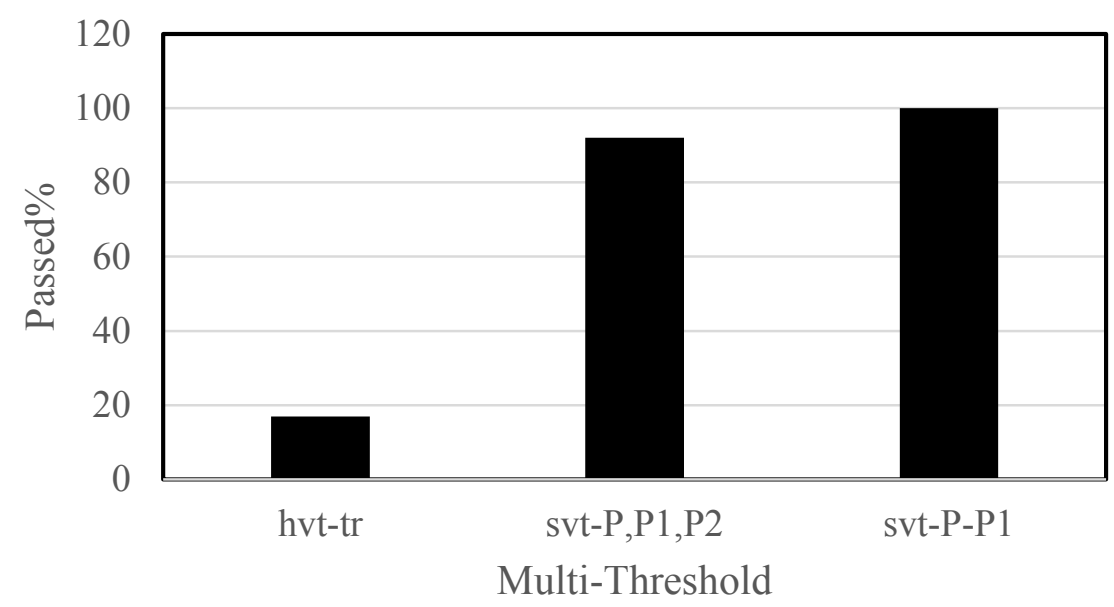

Figure 40 Number of passed of SP flip-flop for different devices

( $V_{d d}=200 \mathrm{mV}, 65 \mathrm{~nm}$ process, hvt devices).

For this case, the rise time of output is $6.01 \mu \mathrm{s}$ and fall time is $6.89 \mu \mathrm{s}$. As a result, SP flip-flop operates properly by producing a $50 \%$ duty cycle output pulse.

\subsection{Comparison between TG and SP flip-flops}

Power consumption becomes larger with increasing supply voltage. The difference between total power and dynamic power is static power. As shown in Figure 41, the power of SP flip-flop is less than TG flip-flop due to fewer transistor count resulting in less current.

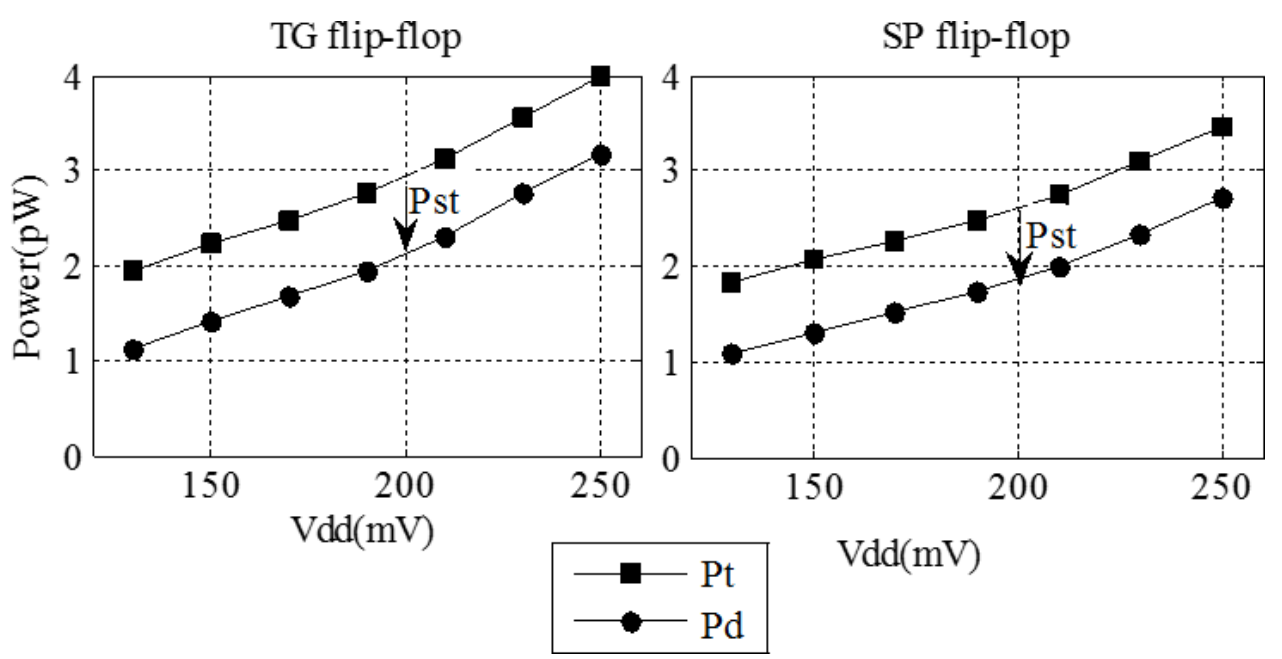

Figure 41 Total, static and dynamic power of TG and SP flip-flop versus $V_{d d}$ ( $T=100 \mu \mathrm{s}, 65 \mathrm{~nm}$ process, multi-threshold devices). 
According to Equation (2-1), dynamic power is proportional to frequency. This relationship is presented in Figure 42. The plots of Figure 42 also show that both subthreshold flip-flops operate for clock period larger than $50 \mu \mathrm{s}$. Therefore, the maximum operating frequency for both configurations is $20 \mathrm{KHz}$ using $V_{d d}=200 \mathrm{mV}$.

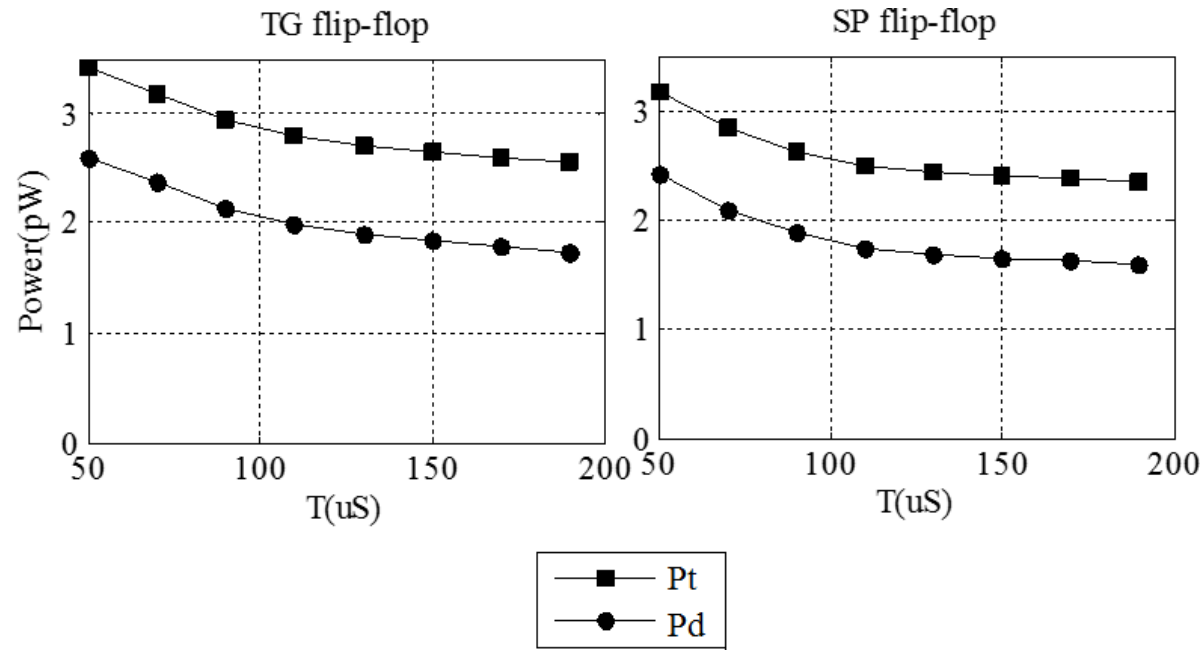

Figure 42 Total, static and dynamic power of TG and SP flip-flop versus $T$ ( $V_{d d}=200 \mathrm{mV}, 65 \mathrm{~nm}$ process, multi-threshold devices).

From Equation (2-22), as $V_{d d}$ increases delay decreases. As shown in Figure 43, falling and rising delay for both transistors are close. Furthermore, SP flip-flop has less delay compared to TG flip-flop as shown in Figure 43.

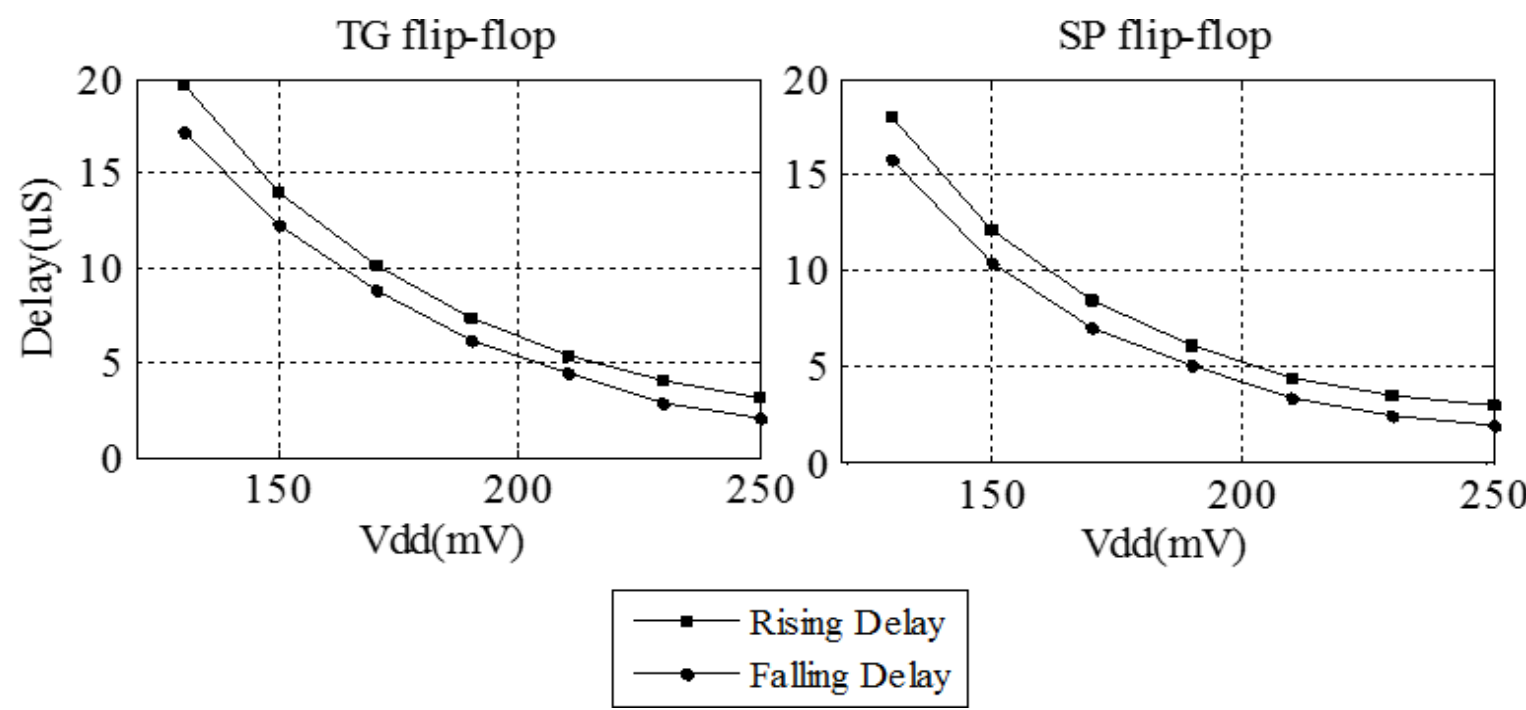

Figure $43 T_{c-q}$ of TG and SP flip-flop versus $V_{d d}(T=100 \mu \mathrm{s}, 65 \mathrm{~nm}$ process, multi-threshold devices). 
From Equation (2-28), when $V_{d d}$ decrease, total energy decrease quadratically. The minimum energy point with regard to the supply voltage is shown in Figure 44.

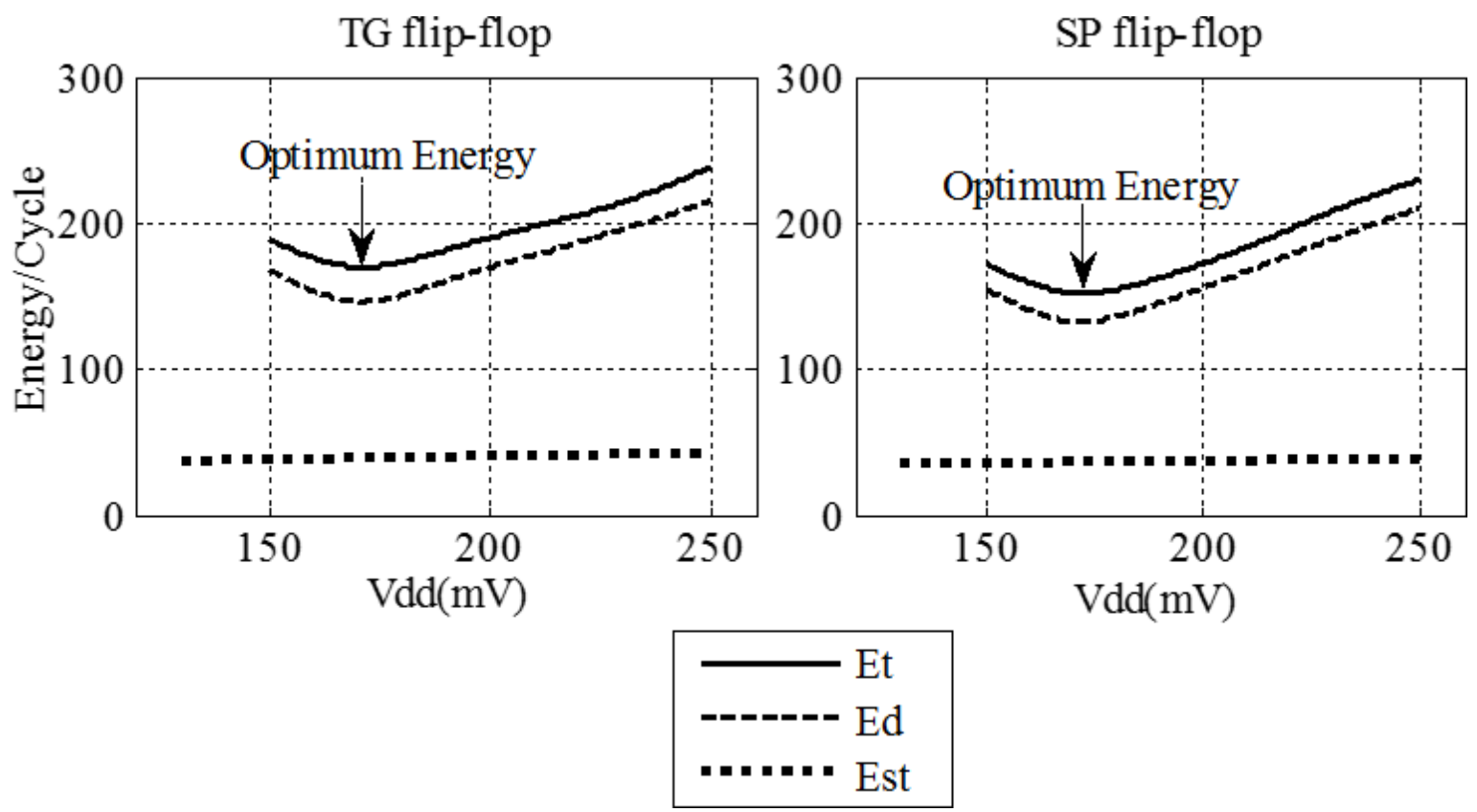

Figure 44 Energy per cycle versus $V_{d d}(65 \mathrm{~nm}$ process, multi-threshold devices).

From Equation (2-3) and (2-28), static energy is proportional to $1 / I_{0} e^{\frac{V g s-V_{t h}}{n V_{T}}}$ $\left(1-e^{-\frac{V_{d s}}{V_{T}}}\right)$. Therefore, static energy increases for larger values of $V_{d s}$. Since static energy relates to drain-source voltage exponentially, the increase of static energy is not significant as seen in Figure 44. Moreover, as explained in section 2.6.5 and shown in Figure 44, there is minimum point energy regard to supply voltage. This minimum energy point for both flip-flops occurs at $V_{d d}=170 \mathrm{mV}$. Table 8 shows the TG and SP flip-flop parameters extracted from simulation results of using $V_{d d}=200 \mathrm{mV}$. Table 9 shows these design parameters using a supply voltage of $170 \mathrm{mV}$, which yields the least energy dissipation. 
Table 8 The simulation results of flip-flops $\left(V_{d d}=200 \mathrm{mV}, T=100 \mu \mathrm{s}, 65 \mathrm{~nm}\right.$ process, multi-threshold devices).

\begin{tabular}{|l|l|l|l|l|l|l|}
\hline & $\begin{array}{l}\mathbf{P}_{\text {stav }} \\
(\mathbf{p W})\end{array}$ & $\begin{array}{l}\mathbf{P}_{\mathbf{d}} \\
(\mathbf{p W})\end{array}$ & $\begin{array}{l}\mathbf{P}_{\text {total }} \\
(\mathbf{p W})\end{array}$ & $\begin{array}{l}\mathbf{T}_{\mathrm{C}-\mathrm{Q}} \\
(\boldsymbol{\mu} \mathbf{s})\end{array}$ & $\begin{array}{l}\mathbf{T}_{\text {setup }} \\
(\boldsymbol{\mu} \mathbf{s})\end{array}$ & $\begin{array}{l}\mathbf{f}_{\max } \\
(\mathbf{K H z})\end{array}$ \\
\hline TG flip-flop & 0.81 & 2.1 & 2.91 & 6.31 & 4.1 & 45.5 \\
\hline SP flip-flop & 0.75 & 1.86 & 2.61 & 5.13 & 3.9 & 50 \\
\hline
\end{tabular}

Table 9. The simulation results of flip-flops $\left(V_{d d}=170 \mathrm{mV}, T=100 \mu \mathrm{s}, 65 \mathrm{~nm}\right.$ process, multi-threshold devices).

\begin{tabular}{|l|l|l|l|l|l|}
\hline & $\begin{array}{l}\mathbf{P}_{\text {stav }} \\
(\mathbf{p W})\end{array}$ & $\begin{array}{l}\mathbf{P}_{\mathbf{d}} \\
(\mathbf{p W})\end{array}$ & $\begin{array}{l}\mathbf{P}_{\text {total }} \\
(\mathbf{p W})\end{array}$ & $\begin{array}{l}\mathbf{T}_{\mathbf{C}-\mathbf{Q}} \\
(\boldsymbol{\mu} \mathbf{s})\end{array}$ & $\begin{array}{l}\mathbf{f}_{\max } \\
(\mathbf{K H z})\end{array}$ \\
\hline TG flip-flop & 0.79 & 1.68 & 2.48 & 10.09 & 14.5 \\
\hline SP flip-flop & 0.73 & 1.52 & 2.25 & 8.39 & 16 \\
\hline
\end{tabular}

\subsection{Effect of Temperature and supply Voltage Variations}

Table 10 shows the mean, sigma, relative sigma, minimum and maximum delay values for TG and SP flip-flops at different temperatures and supply voltages. As shown in Table 10, the yield of both flip-flops are not $100 \%$ at $-40{ }^{\circ} \mathrm{C}$. The transistor subthreshold current decreases as the temperature lowers according to Equation (2-1), thus slowing the transistors. However, SP flip-flop shows a higher yield for $T=-40{ }^{\circ} \mathrm{C}$. Furthermore, the dispersion of delay values (б) is almost the same for both flip-flops. Therefore, according to mean, sigma, $\sigma, D_{\min }$, and $D_{M a x}$, the reliability of SP flip-flop is almost the same as TG structure with respect to PVT variations. However, SP flip-flop consumes less power and thus is superior over TG.

Table 10. Mean, Sigma, minimum, and maximum delay of TG and SP flip-flop $(T=100 \mathrm{us,} 65 \mathrm{~nm}$ process, multi-threshold devices).

\begin{tabular}{|l|c|c|c|}
\hline \multirow{2}{*}{$V_{d d}=\mathbf{2 2 0 m}$} & \multicolumn{3}{|c|}{$T=80^{\circ} \mathrm{C}$} \\
\cline { 2 - 4 } & $T=-40^{\circ} \mathrm{C}$ & $T=27^{\circ} \mathrm{C}$ & $T=80^{-}$ \\
\hline
\end{tabular}




\begin{tabular}{|c|c|c|c|c|}
\hline \multirow{20}{*}{$\begin{array}{c}\text { TG } \\
\text { FF } \\
\text { Delay }\end{array}$} & \multicolumn{2}{|r|}{ Yield $=88 \%$} & \multirow{2}{*}{$\begin{array}{l}\text { Yield }=100 \% \\
8.83 \mu \mathrm{s}\end{array}$} & \multirow{2}{*}{$\begin{array}{l}\text { Yield }=100 \% \\
1.037 \mu \mathrm{s}\end{array}$} \\
\hline & Mean & $40.31 \mu \mathrm{s}$ & & \\
\hline & Sigma & $27.9 \mathrm{~s} \mu$ & $6.27 \mu \mathrm{s}$ & $0.65 \mu \mathrm{s}$ \\
\hline & $\sigma$ & 0.69 & 0.7 & 0.62 \\
\hline & $D_{\min }$ & $2.03 \mu \mathrm{s}$ & $0.93 \mu \mathrm{s}$ & $0.14 \mu \mathrm{s}$ \\
\hline & $D_{\operatorname{Max}}$ & $101.3 \mu \mathrm{s}$ & $43.91 \mu \mathrm{s}$ & $4.51 \mu \mathrm{s}$ \\
\hline & \multicolumn{4}{|c|}{$V_{d d}=200 \mathrm{~m}$} \\
\hline & \multicolumn{2}{|r|}{$\begin{array}{l}T=-40{ }^{\circ} \mathrm{C} \\
\text { Yield }=88 \%\end{array}$} & $\begin{array}{l}T=27^{\circ} \mathrm{C} \\
\text { Yield }=100 \%\end{array}$ & $\begin{array}{l}T=80{ }^{\circ} \mathrm{C} \\
\text { Yield }=100 \%\end{array}$ \\
\hline & Mean & $43.71 \mu \mathrm{s}$ & $8.18 \mu \mathrm{s}$ & $0.96 \mu \mathrm{s}$ \\
\hline & Sigma & $30.91 \mu \mathrm{s}$ & $5.8 \mu \mathrm{s}$ & $0.61 \mu \mathrm{s}$ \\
\hline & 6 & 0.7 & 0.7 & 0.62 \\
\hline & $D_{\text {min }}$ & $2.82 \mu \mathrm{s}$ & $0.87 \mu \mathrm{s}$ & $0.13 \mu \mathrm{s}$ \\
\hline & $D_{\operatorname{Max}}$ & $112.2 \mu \mathrm{s}$ & $40.34 \mu \mathrm{s}$ & $4.2 \mu \mathrm{s}$ \\
\hline & \multicolumn{4}{|c|}{$V_{d d}=180 \mathrm{~m}$} \\
\hline & & $\begin{array}{l}T=-40{ }^{\circ} \mathrm{C} \\
\text { Yield }=75 \%\end{array}$ & $\begin{array}{l}T=27^{\circ} \mathrm{C} \\
\text { Yield }=100 \%\end{array}$ & $\begin{array}{l}T=80^{\circ} \mathrm{C} \\
\text { Yield }=100 \%\end{array}$ \\
\hline & Mean & $45.12 \mu \mathrm{s}$ & $7.55 \mu \mathrm{s}$ & $0.89 \mu \mathrm{s}$ \\
\hline & Sigma & $34.15 \mu \mathrm{s}$ & $5.39 \mu \mathrm{s}$ & $0.56 \mu \mathrm{s}$ \\
\hline & $\sigma$ & 0.75 & 0.7 & 0.62 \\
\hline & $D_{\min }$ & $4.66 \mu \mathrm{s}$ & $0.82 \mu \mathrm{s}$ & $0.12 \mu \mathrm{s}$ \\
\hline & $D_{\operatorname{Max}}$ & $124.1 \mu \mathrm{s}$ & $36.91 \mu \mathrm{s}$ & $3.89 \mu \mathrm{s}$ \\
\hline \multirow{21}{*}{$\begin{array}{c}\text { SP } \\
\text { FF } \\
\text { Delay }\end{array}$} & \multicolumn{4}{|c|}{$V_{d d}=220 \mathrm{~m}$} \\
\hline & \multicolumn{2}{|r|}{$\begin{array}{l}T=-40{ }^{\circ} \mathrm{C} \\
\text { Yield }=92 \%\end{array}$} & $\begin{array}{l}T=27{ }^{\circ} \mathrm{C} \\
\text { Yield }=100 \%\end{array}$ & $\begin{array}{l}T=80^{\circ} \mathrm{C} \\
\text { Yield }=100 \%\end{array}$ \\
\hline & Mean & $28.3 \mu \mathrm{s}$ & $5.18 \mu \mathrm{s}$ & $0.7 \mu \mathrm{s}$ \\
\hline & Sigma & $19.8 \mu \mathrm{s}$ & $3.6 \mu \mathrm{s}$ & $0.43 \mu \mathrm{s}$ \\
\hline & $\sigma$ & 0.7 & 0.68 & 0.61 \\
\hline & $D_{\text {min }}$ & $1.82 \mu \mathrm{s}$ & $0.66 \mu \mathrm{s}$ & $0.12 \mu \mathrm{s}$ \\
\hline & $D_{\operatorname{Max}}$ & $99.3 \mu \mathrm{s}$ & $25.06 \mu \mathrm{s}$ & $3.16 \mu \mathrm{s}$ \\
\hline & \multicolumn{4}{|c|}{$V_{d d}=200 \mathrm{~m}$} \\
\hline & \multicolumn{2}{|r|}{$\begin{array}{l}T=-40{ }^{\circ} \mathrm{C} \\
\text { Yield }=90 \%\end{array}$} & $\begin{array}{l}T=27^{\circ} \mathrm{C} \\
\text { Yield }=100 \%\end{array}$ & $\begin{array}{l}T=80^{\circ} \mathrm{C} \\
\text { Yield }=100 \%\end{array}$ \\
\hline & Mean & $30.9 \mu \mathrm{s}$ & $6.83 \mu \mathrm{s}$ & $0.85 \mu \mathrm{s}$ \\
\hline & Sigma & $21.3 \mu \mathrm{s}$ & $4.77 \mu \mathrm{s}$ & $0.52 \mu \mathrm{s}$ \\
\hline & 6 & 0.69 & 0.68 & 0.61 \\
\hline & $D_{\min }$ & $2.29 \mu \mathrm{s}$ & $0.79 \mu \mathrm{s}$ & $0.13 \mu \mathrm{s}$ \\
\hline & $D_{\operatorname{Max}}$ & $99.6 \mu \mathrm{s}$ & $29.88 \mu \mathrm{s}$ & $3.16 \mu \mathrm{s}$ \\
\hline & \multicolumn{4}{|c|}{$V_{d d}=180 \mathrm{~m}$} \\
\hline & \multicolumn{2}{|r|}{$\begin{array}{l}T=-40^{\circ} \mathrm{C} \\
\text { Yield }=87 \%\end{array}$} & $\begin{array}{l}T=27{ }^{\circ} \mathrm{C} \\
\text { Yield }=100 \%\end{array}$ & $\begin{array}{l}T=80{ }^{\circ} \mathrm{C} \\
\text { Yield }=100 \%\end{array}$ \\
\hline & Mean & $35.78 \mu \mathrm{s}$ & $9.46 \mu \mathrm{s}$ & $1.09 \mu \mathrm{s}$ \\
\hline & Sigma & $25.56 \mu \mathrm{s}$ & $6.86 \mu \mathrm{s}$ & $0.68 \mu \mathrm{s}$ \\
\hline & 6 & 0.71 & 0.72 & 0.62 \\
\hline & $D_{\text {min }}$ & $3.6 \mu \mathrm{s}$ & $1.037 \mu \mathrm{s}$ & $0.16 \mu \mathrm{s}$ \\
\hline & $D_{\operatorname{Max}}$ & $103.7 \mu \mathrm{s}$ & $45.95 \mu \mathrm{s}$ & $4.61 \mu \mathrm{s}$ \\
\hline
\end{tabular}




\subsection{Transmission Gate Flip-Flop Layout}

Appendix B presents the layout and extracted view of TG flip-flop. Since interconnect capacitance and parasitic effects cannot be modelled in simulation, one of the proposed flip-flops is extracted in $65 \mathrm{~nm}$ process for this thesis. Furthermore, the layout and schematic simulation results are compared. As shown in Figure B.3 and Table (B.1), the power consumption increases by almost $53 \%$ and delay by $38 \%$ for the extracted view due to additional parasitic. Moreover, Figure B.4 presents the Monte Carlo simulation results conducted with the extracted layout of TG flip-flop, showing decreased reliability compared to schematic simulation.

\subsection{Summary}

In this chapter, we have presented the different building blocks of flip-flops: Inverter, tristate inverter and transmission gate inverter. Two flip-flop topologies, transmission gate and single phase configurations, were analyzed for reliability, power and delay considerations using the $65 \mathrm{~nm}$ process. It was shown that tri-state inverters show superior delay and reliability over other flip-flop building blocks. As a result, they were employed in the feedback path of TG flip-flops. Furthermore, hvt- and svt-transistors were tested for reliability within both TG and SP flip-flops using Monte Carlo simulations. The most reliable configurations using minimum size hvt- and svt- transistors were used for both flip-flop structures. Moreover, Monte Carlo simulations were used to compare relative sigma values using different temperature and supply voltages for the TG and SP flip-flops. It was observed that both flip-flops have almost the same reliability. In addition, these flip-flops were analyzed for power consumption. It was observed that the SP flip-flops consume less power, and as a result are superior over TG flip-flops.

In the next chapter, an improved device characterization technique is presented while focusing on lower power and higher reliability designs using a $28 \mathrm{~nm}$ process. 


\section{Chapter 6. Improved Device Characterization technique}

Recently, Canadian academia obtained access to the STM FD-SOI 28 nm CMOS design technology kit. After that, we decided to update and pursue the research on the new technology rather than the older $65 \mathrm{~nm}$ technology. This move felt a necessity because the behavior of transistors in newer CMOS technologies (with shorter feature lengths) is typically less ideal. Hence, circuit design in these technologies is more challenging and more appealing to the ICs community. For this part of the work, the CAD tool used is Cadence, the simulation engine is Spectre, the transistor models are based on BSIMSOI, and the technology kit is STM $28 \mathrm{~nm}$ FD-SOI.

As we had to repeat the device characterization for the $28 \mathrm{~nm}$ technology, we thought of a novel approach to do so. Instead of plotting the behavior of a MOSFET versus its length and width separately in 2D, we found it is more convenient and more in-formative if we combine the two graphs into a single $3 \mathrm{D}$ one. To do so, the value of the current is obtained by sweeping the channel length and width simultaneously and the results are displayed in a $3 \mathrm{D}$ contour graph. However, since the current changes significantly in the small transistor sizes of interest, a logarithmic scale had to be used for the current.

Such a graph allows the designer to choose appropriate transistor dimensions for obtaining a particular amount of operational or idle current. At the same time, the designer would have an idea of the relative amount of the capacitance the transistor imposes within a circuit. Therefore, the contour graph allows for balancing between the design metrics faster and more conveniently compared to exhaustive blind circuit simulations. The final tuning can be still made with few circuit simulations.

\subsection{Transistors ON-Current Contour Plots}

According to Equation (2-3), the drain current in the subthreshold region alters due to changes in the width and length of a transistor. However, the actual subthreshold current behavior is much more complicated than the equation implies. This is because of the inter- 
dependency of some of the parameters in the current equation and some factors such the INWE that the equation doesn't account for. The contour plots in Figure 45 and Figure 46 show the subthreshold current behavior versus $W$ and $L$ for svt-NMOS ( $V_{t h}=400.4 \mathrm{mV}$ ) and svt-PMOS $\left(V_{t h}=-518.3 \mathrm{mV}\right)$ devices in the $28 \mathrm{~nm}$ technology, respectively.

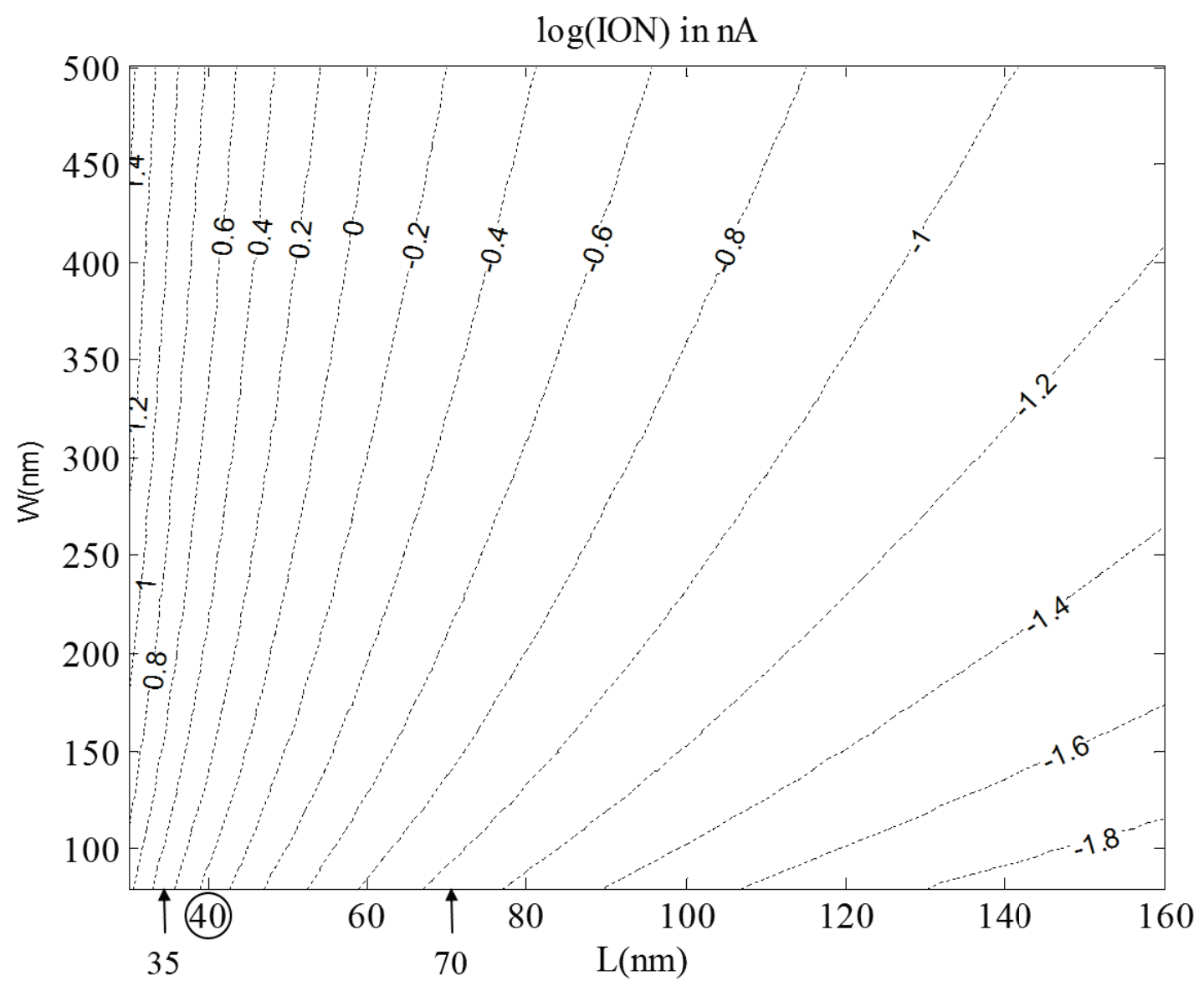

Figure $45 I_{o N}$ versus $L$ and $W\left(V_{d d}=200 \mathrm{mV}, 28 \mathrm{~nm}\right.$ process, svt-NMOS). 


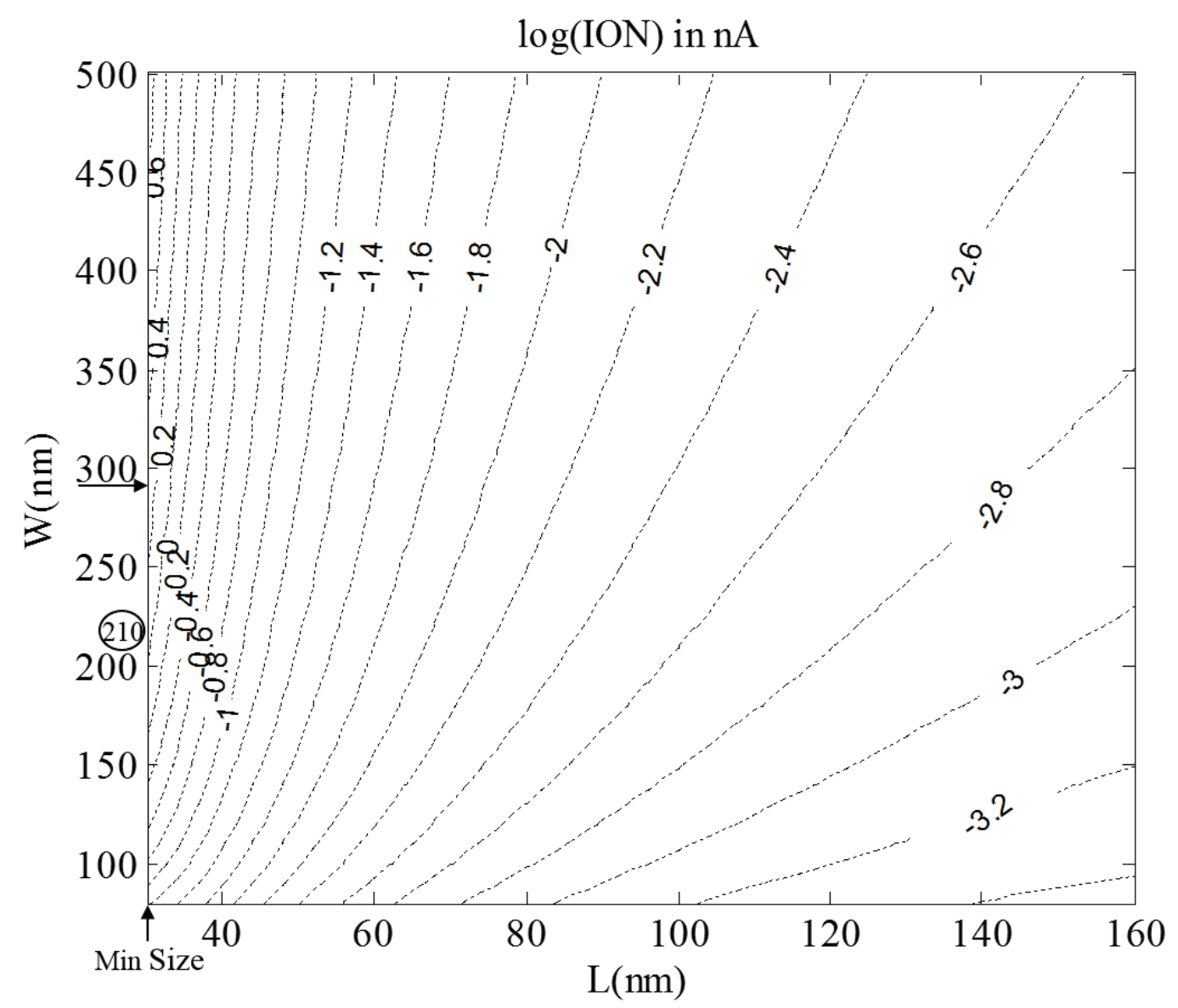

Figure $46 I_{o N}$ versus $L$ and $W\left(V_{d d}=200 \mathrm{mV}, 28 \mathrm{~nm}\right.$ process, svt-PMOS $)$.

From these graphs, for example, we can easily find that in order to implement the smallest inverter with a symmetrical rising and falling output transitions, the PMOS device should be $210 \mathrm{~nm}$ wide with the minimum length of $30 \mathrm{~nm}$, while the NMOS device should be $40 \mathrm{~nm}$ long with the minimum width of $80 \mathrm{~nm}$. These points are circled in Figure 45 and Figure 46 and both indicate a current of $1 \mathrm{~nm}$. These dimensions are not predictable by the usual rule of using the minimum channel length and making the PMOS device twice as wide as the NMOS device. They cannot also be easily found through ad-hoc simulations.

\subsection{Transistors OFF-Current Contour Plots}

The OFF-current, i.e. the subthreshold leakage current when a transistor if OFF, is a notion of the static (or idle) power dissipation. The contour plots in Figure 47 and Figure 48 
show the subthreshold current behavior versus $W$ and $L$ for NMOS and PMOS devices in the $28 \mathrm{~nm}$ technology, respectively.

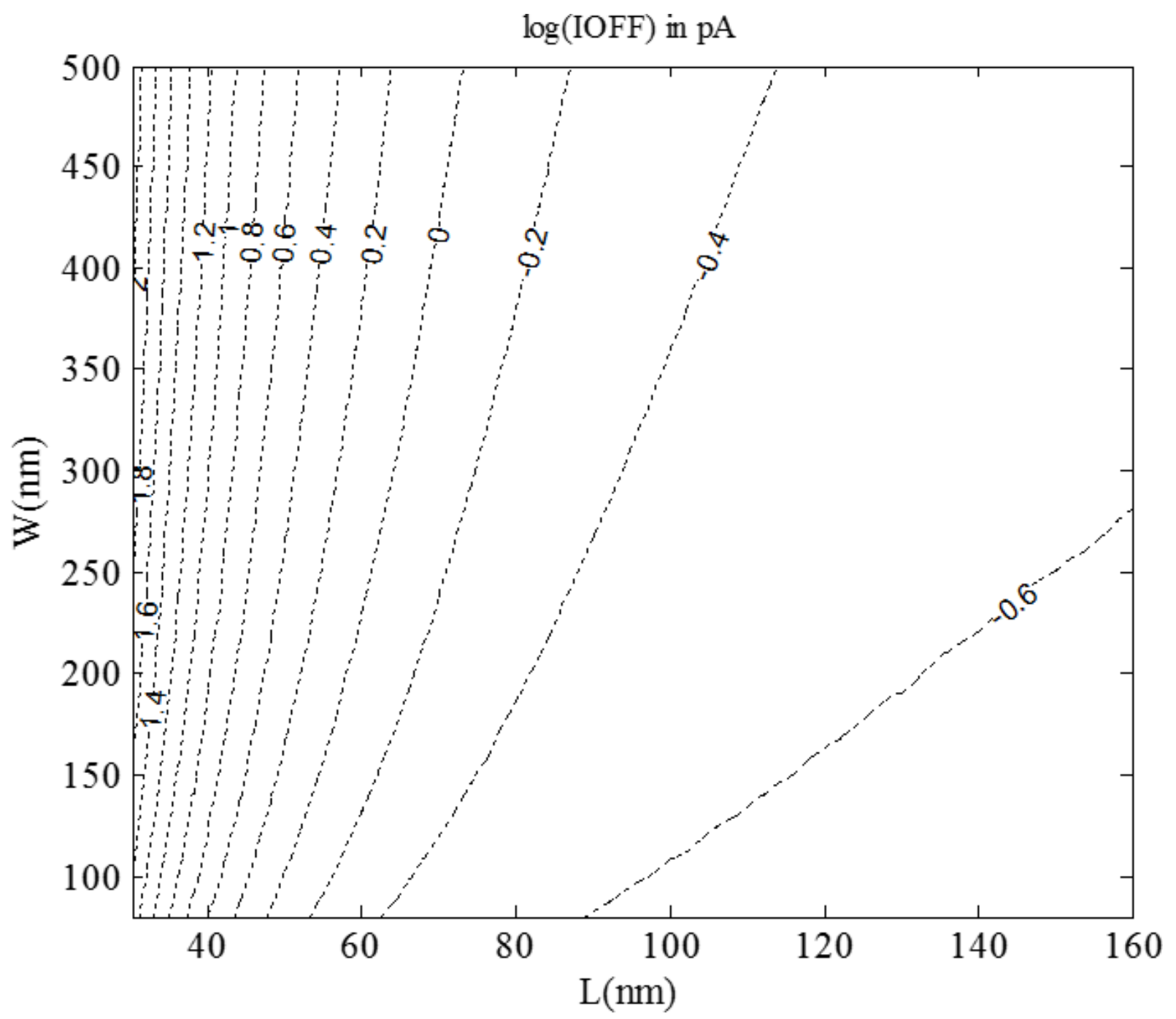

Figure $47 I_{\text {oFF }}$ versus $L$ and $W\left(V_{d d}=200 \mathrm{mV}, 28 \mathrm{~nm}\right.$ process, svt-NMOS $)$. 


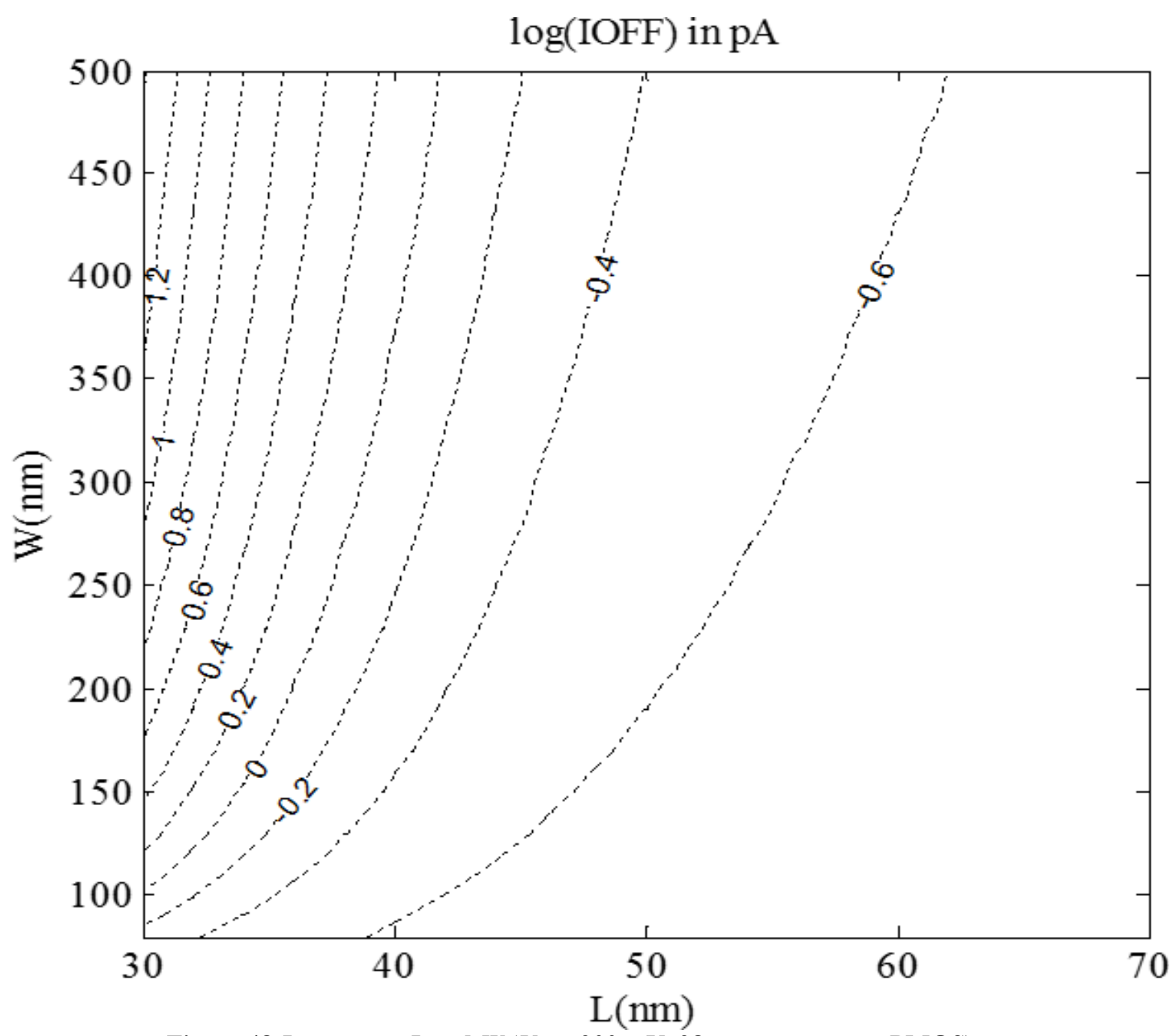

Figure 48 Ioff versus $L$ and $W\left(V_{d d}=200 \mathrm{mV}, 28 \mathrm{~nm}\right.$ process, svt-PMOS).

\subsection{Relative Sigma Contour Plots}

To assess the reliability of a device, evaluating the relative sigma (i.e. ratio of the standard deviation to the mean) seems the best option (from Monte Carlo simulations) that shows the extent of variability in relation to the mean of the population. Figure 49 and Figure 50 shows the contour plot of the relative sigma of NMOS and PMOS transistors in the $28 \mathrm{~nm}$ technology, respectively. 


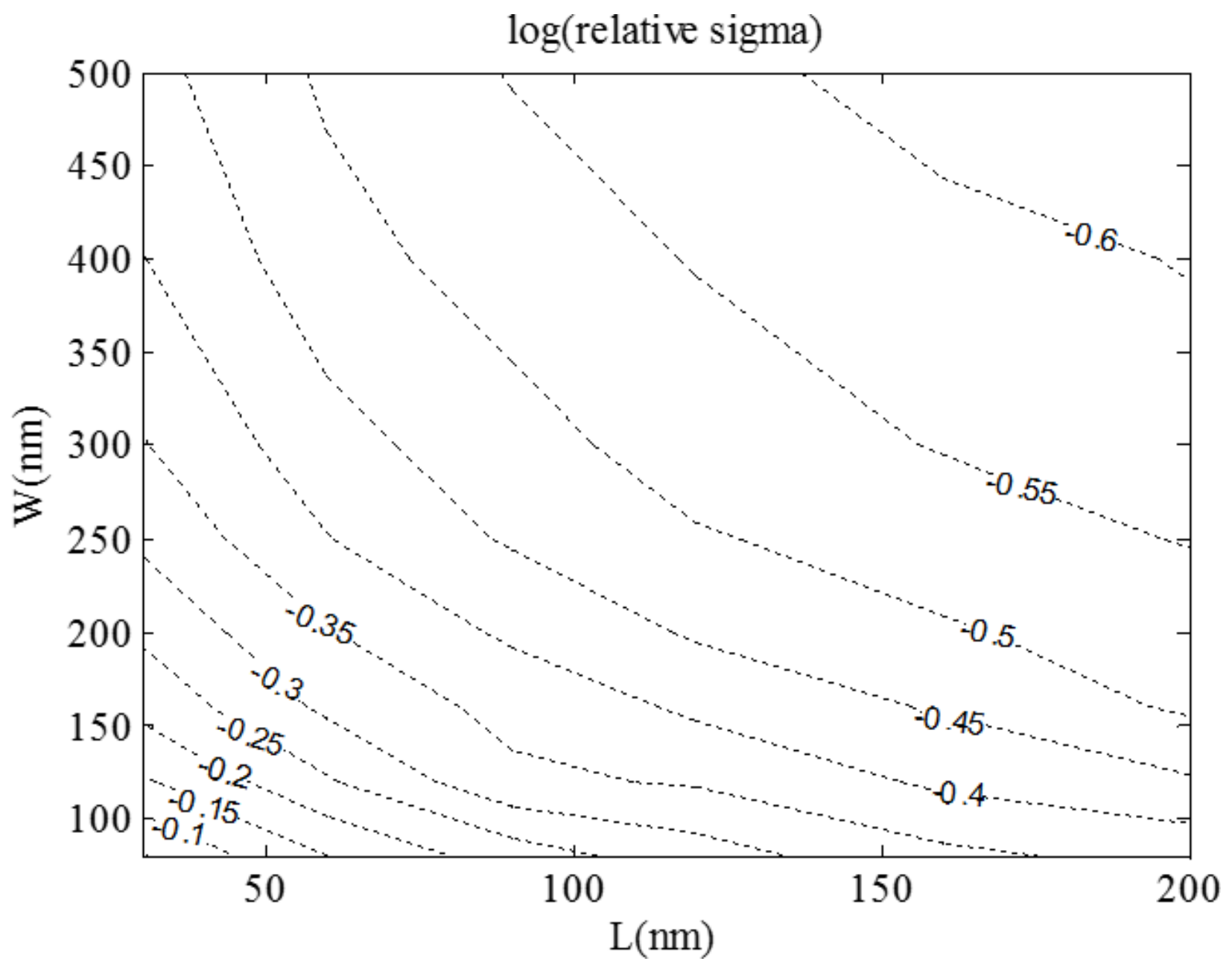

Figure 49 Relative sigma of NMOS ON-current ( $V_{d d}=200 \mathrm{mV}, 28 \mathrm{~nm}$ process). 


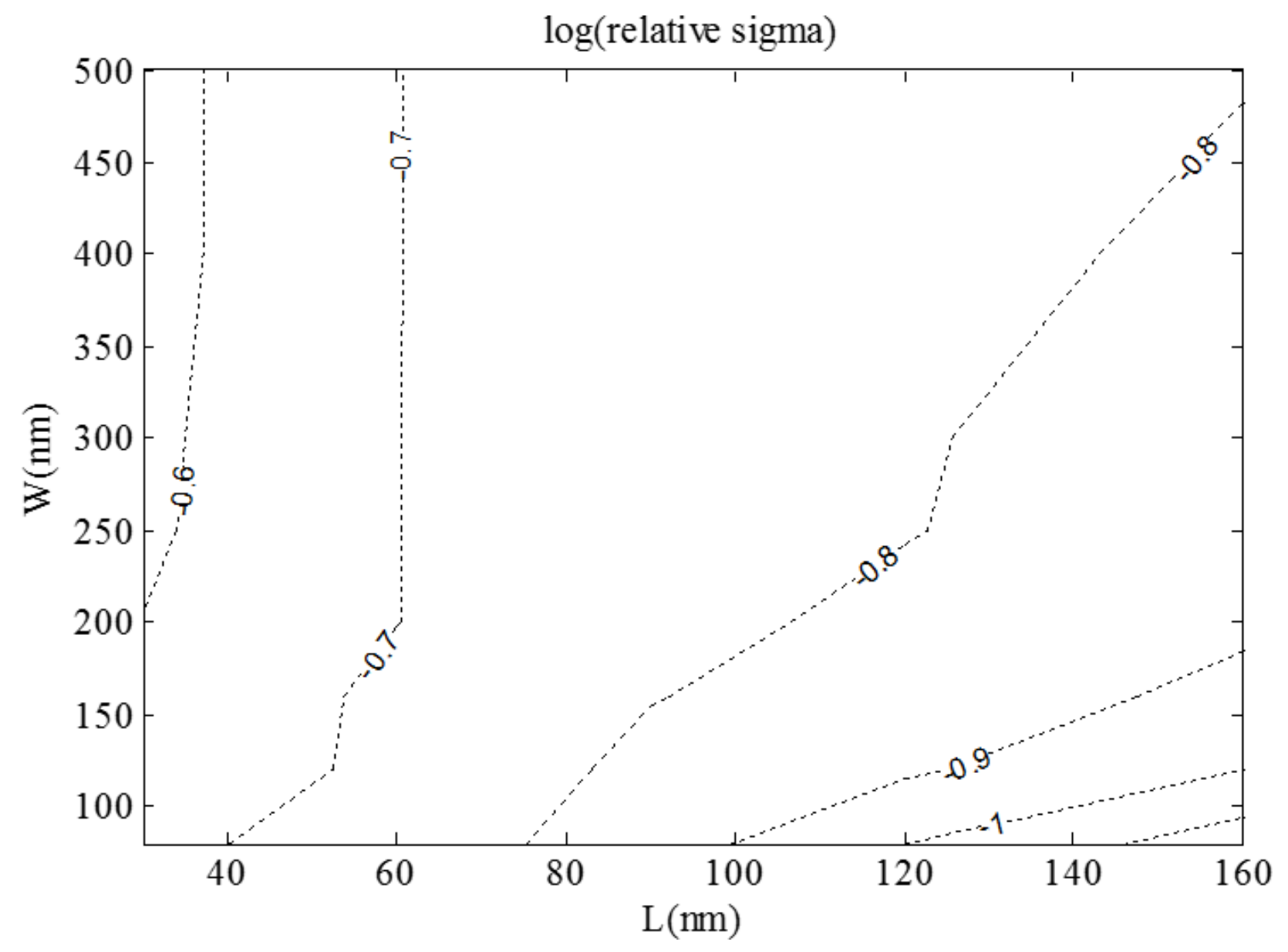

Figure 50 Relative sigma of PMOS ON-current ( $V_{d d}=200 \mathrm{mV}, 28 \mathrm{~nm}$ process).

From Figure 49 and Figure 50, we can find the how values are dispersed around the mean. Generally, relative sigma of transistors get smaller as they become wider and longer. Hence, as expected larger transistors are more reliable. However, the PMOS transistor in this technology seem to be an exception, as for a given channel length, its ON-current variations (evaluated by relative sigma) increase with increasing the width.

\subsection{Low Threshold Voltage Transistors ON and OFF-Current Con- tour Plots}

The current of the minimum-size low-threshold voltage PMOS transistor is 10 times that of its standard-voltage counterpart. We may need to replace a svt-PMOS with an lvt-PMOS transistor when a higher current drive is needed. The contour plots in Figure 51 and Figure 
52 show the subthreshold current behavior versus $W$ and $L$ for lvt-NMOS ( $V_{t h}=365.9 \mathrm{mV}$ ) and lvt-PMOS $\left(V_{t h}=-454.5 \mathrm{mV}\right)$ devices in the $28 \mathrm{~nm}$ technology, respectively. Figure 53 and Figure 54 show the corresponding OFF-currents.

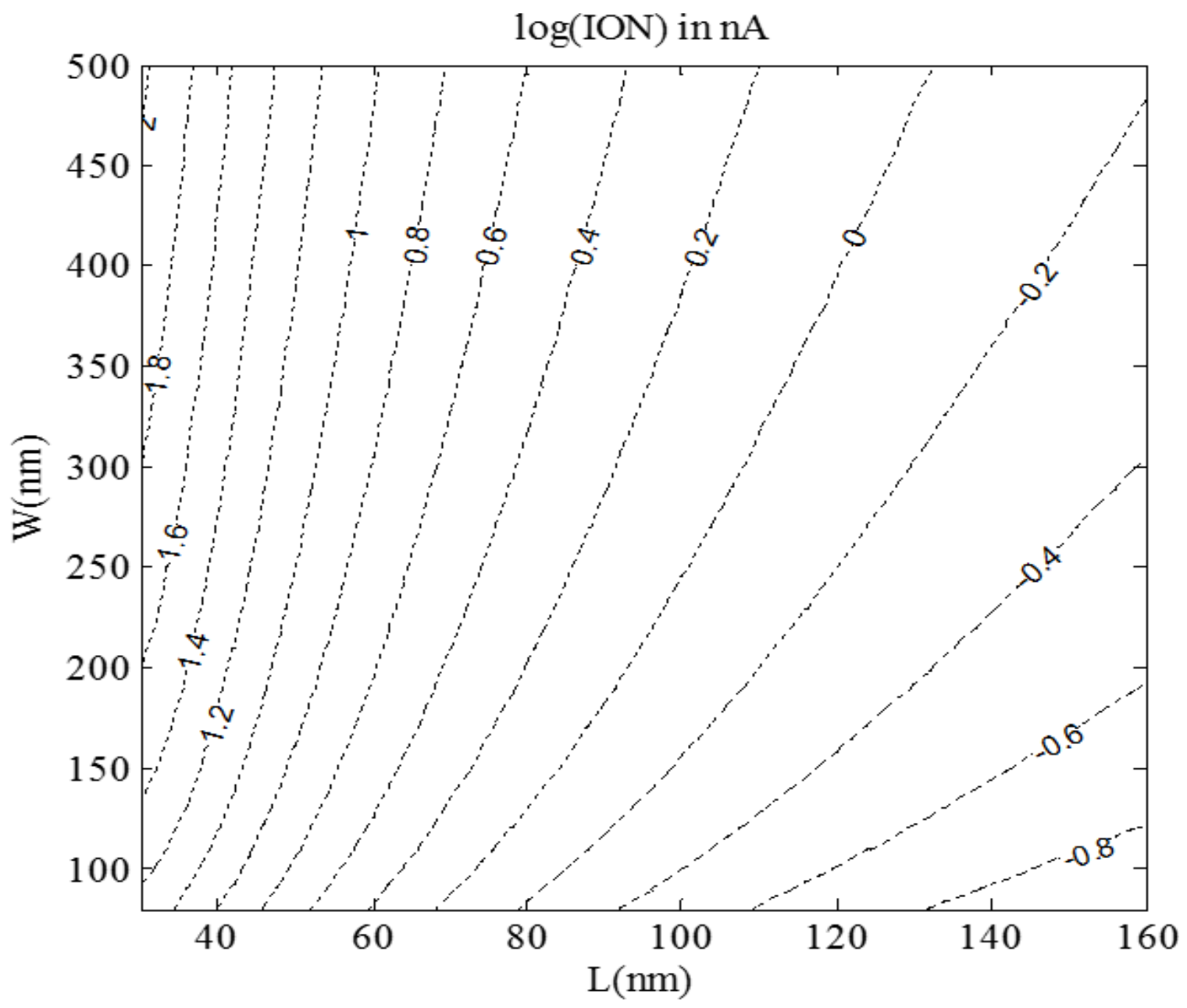

Figure $51 I_{o N}$ versus $L$ and $W\left(V_{d d}=200 \mathrm{mV}, 28 \mathrm{~nm}\right.$ process, lvt-NMOS). 


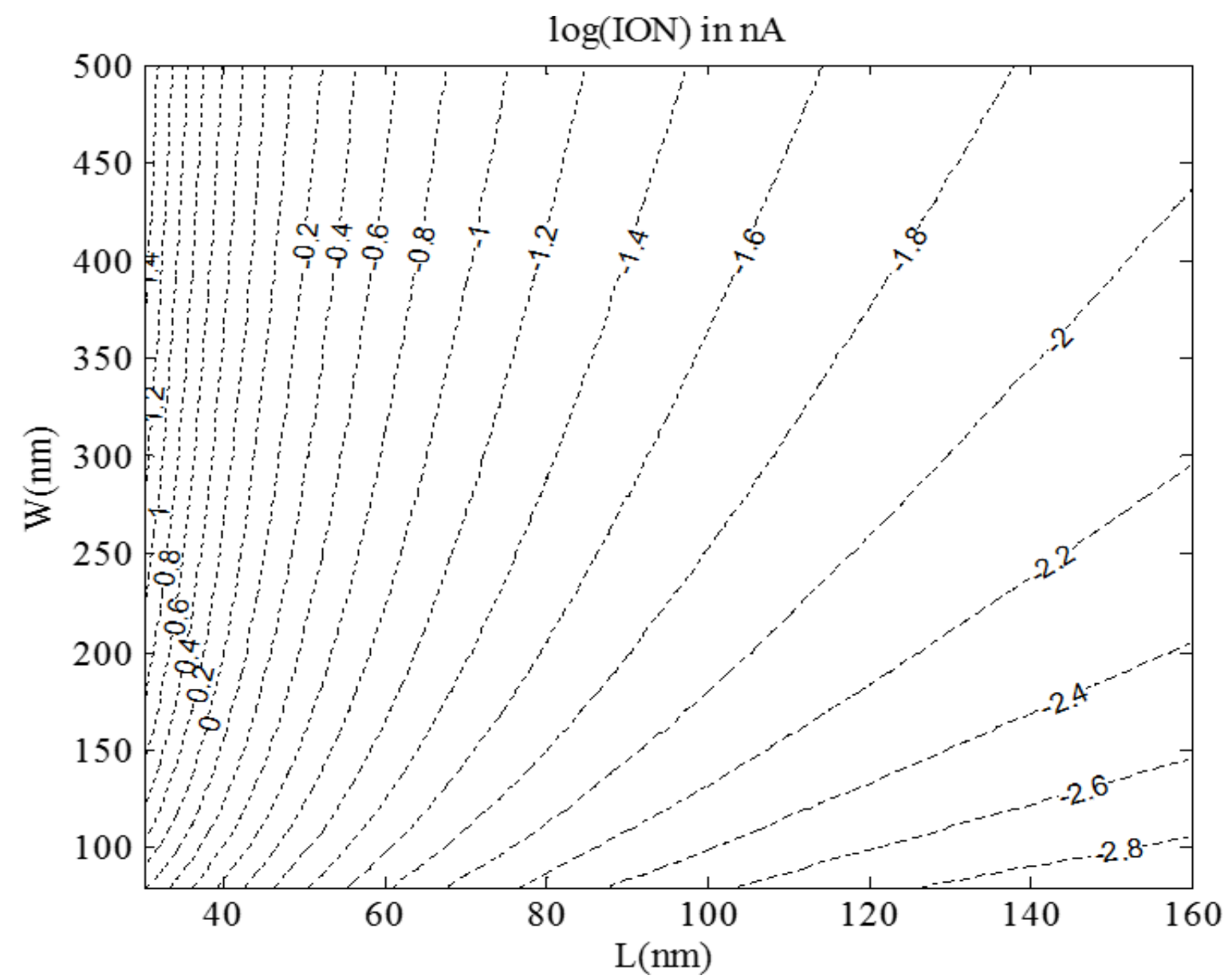

Figure $52 I_{O N}$ versus $L$ and $W\left(V_{d d}=200 \mathrm{mV}, 28 \mathrm{~nm}\right.$ process, lvt-PMOS). 


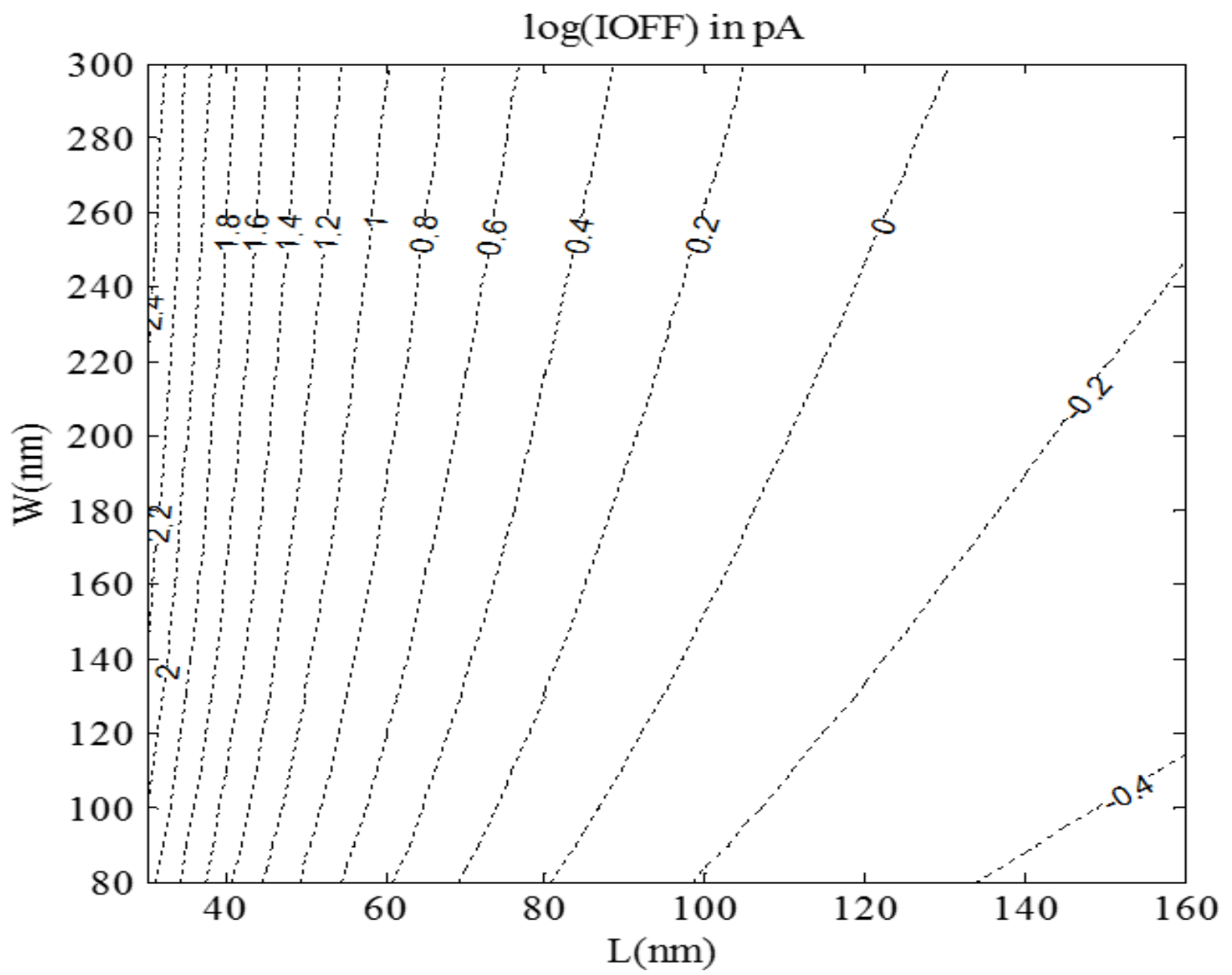

Figure 53 IofF versus $L$ and $W\left(V_{d d}=200 \mathrm{mV}, 28 \mathrm{~nm}\right.$ process, Ivt-NMOS $)$. 


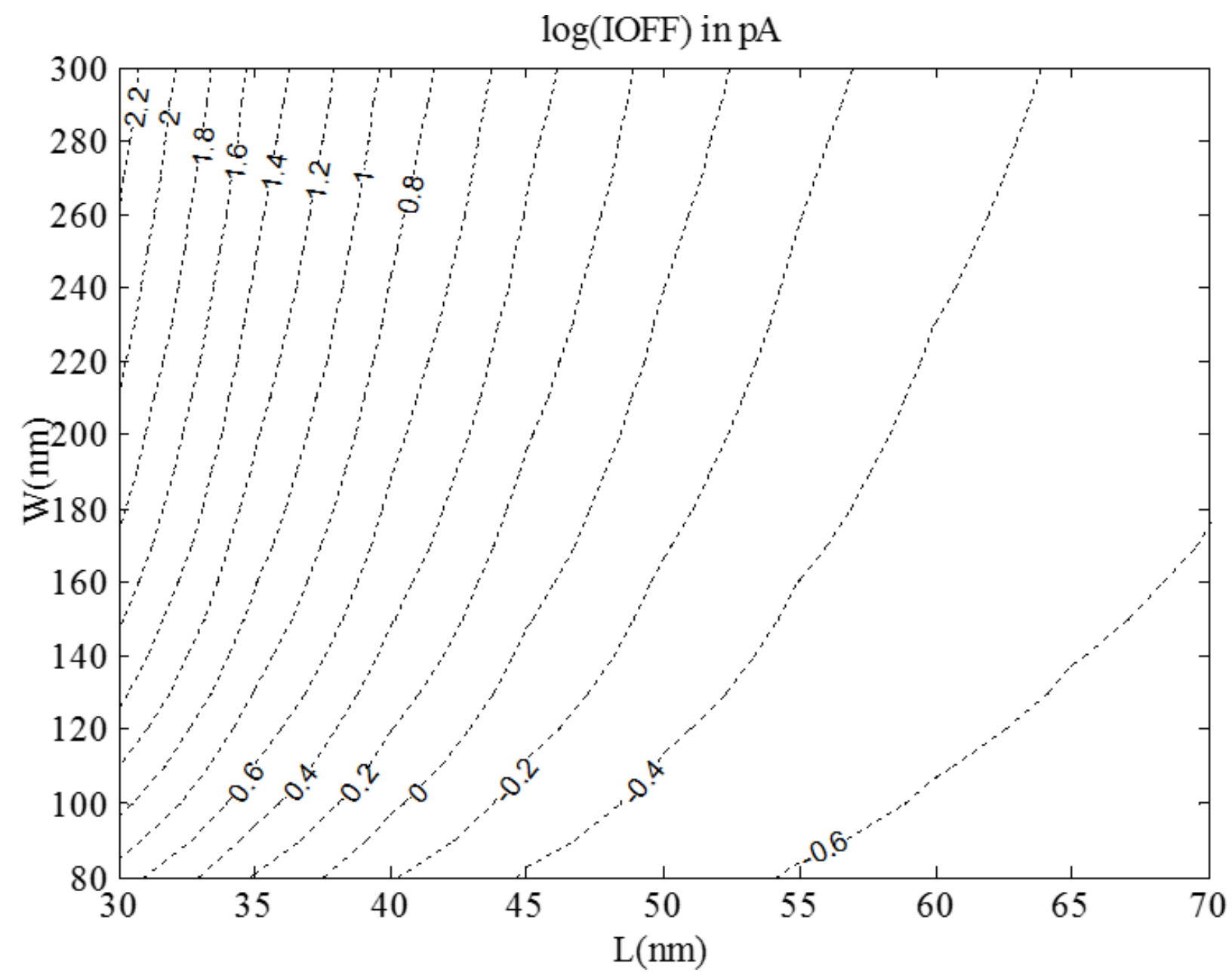

Figure $54 I_{\text {ofF }}$ versus $L$ and $W\left(V_{d d}=200 \mathrm{mV}, 28 \mathrm{~nm}\right.$ process, lvt-PMOS).

\subsection{Summary}

In this chapter, we presented a new technique for transistor sizing by $3 \mathrm{D}$ contour plots using the $28 \mathrm{~nm}$. In the next chapter, this techniques will be applied to design reliable and efficient flip-flops. 


\section{Chapter 7. Reliable Subthreshold Flip-Flop De- sign by transistor Sizing}

In this chapter, we apply the framework presented in Chapter 6 to the design of reliable and efficient flip-flop circuits in the $28 \mathrm{~nm}$ technology dedicated to subthreshold operation.

Beside the test for proper functionality at the typical process case, room temperatrue of $27{ }^{\circ} \mathrm{C}$, and nominal supply voltage of $200 \mathrm{mV}$, we report the simulation results for the circuits under PVT variations. For this purpose, the four processing corners, an extreme cold temperature condition of $-40{ }^{\circ} \mathrm{C}$ and an extreme warm temperature condition of $80{ }^{\circ} \mathrm{C}$, and $\pm 10 \%$ variations in the supply voltage (i.e. $V_{d d}=180 \mathrm{mv}$ and $V_{d d}=220 \mathrm{mv}$ ) are considered.

The delay, power and energy consumption figures for the flip-flop circuits are reported at these temperature and $V_{d d}$ conditions. Monte Carlo simulations are also performed at these conditions to report the dispersion of the circuits' delays. The minimum operational $V_{d d}$ and the minimum-energy $V_{d d}$ for each circuit are also found and reported.

\subsection{Design of PVT-Tolerant Subthreshold Flip-Flops}

In this section, we use transistor sizing technique to improve the yield of TG and SP flipflop based on Monte Carlo simulations. First, we examine the yield of these circuits subjected to process variations when all their transistors are of minimum size (i.e. $\mathrm{L}=30 \mathrm{~nm}$ and $\mathrm{W}=80 \mathrm{~nm}$ ). Minimum-size transistors are commonly used in subthreshold circuits because, often, they guarantee the minimum energy consumption. On the other hand, the TG flip-flop is known to be a ratio-less circuit, i.e. a circuit that functions properly independent of its transistors' sizes [3]. We, however, show that this is only true if the flip-flop is operating in the super-threshold region. The reason is that subthreshold circuits are far more sensitive to process variations that prevents some contentions in the flip-flop to resolve favourably.

Then we use the contour plots of Chapter 6 to adjust the sizes of the critical transistors in the circuits to improve the yield. We visually examine the simulation waveforms 
of the test bench in Figure 27 produced for the TT case and the four process corners FF, SS, FS, and SF. Next, we evaluate the yield by Monte Carlo simulations. The criterion we use to ensure that a flip-flop implementation functions properly, is by asking the CAD tool to count the number of $D, Q$, and clock pulses in the test bench after each Monte Carlo simulation. If the flip-flop is functional, the number of $D$ and $Q$ pulses should be equal and half of the number of clock pulses. Of course, $D, Q$, and internal nodes of the flip-flop are initialized before each round of simulation.

\subsubsection{Transmission Gate Flip-Flop (TG-FF)}

The schematic of the TG-FF is depicted in Figure 55. Circuit simulations show that this circuit in the smallest size implementation only works best in the SF corner, and fails in the FS corner.

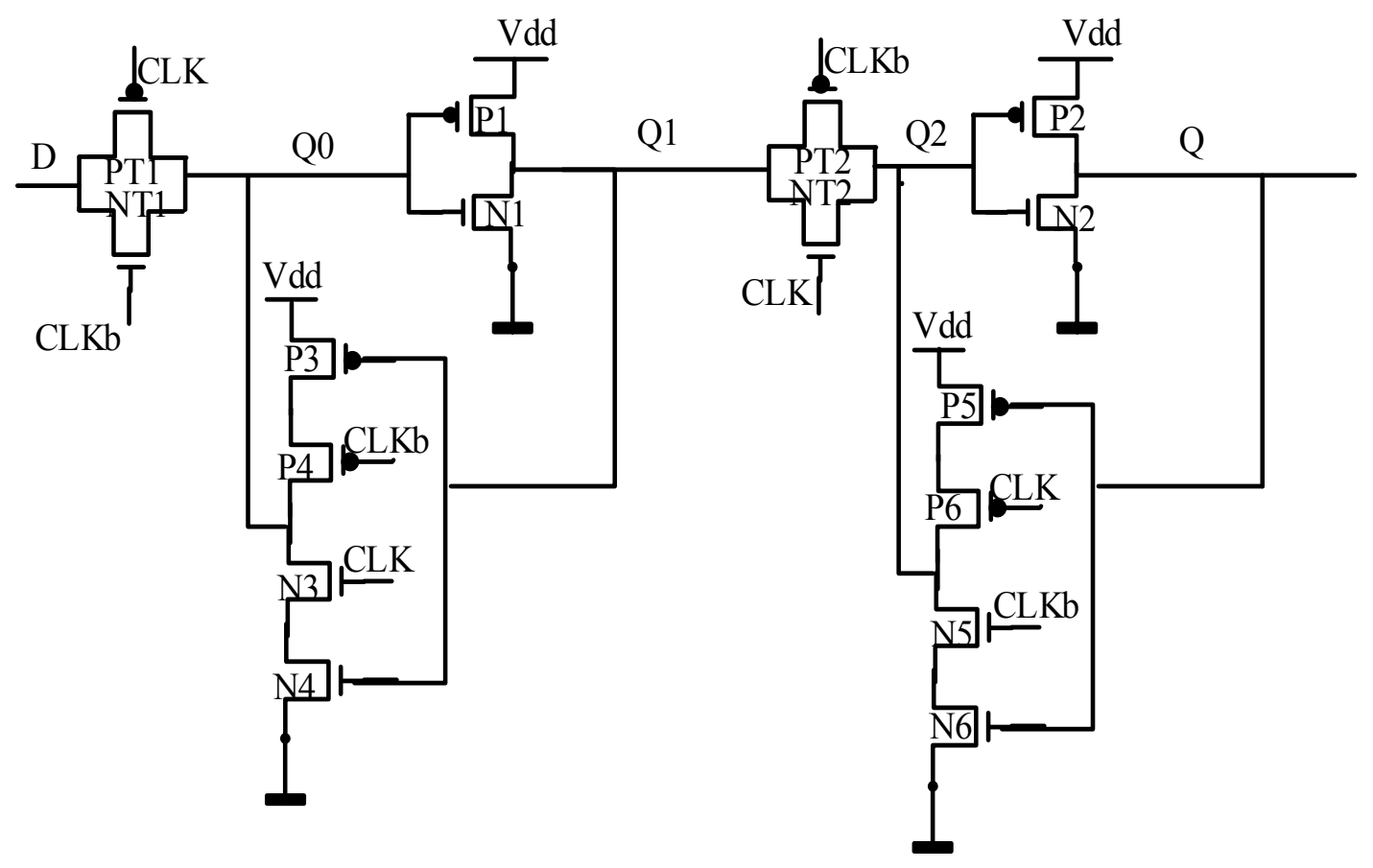

Figure 55 Transmission gate flip-flop schematic. 
Figure 56 shows the timing diagram of signals $C L K, D$, and $Q$ in the FS (top waveforms) and SF (bottom waveforms) corners. In the SF corner, $Q$ is an inverted version of $D$ and follows it properly. The frequency of $D$ and $Q$ is also, as expected, half the $C L K$ frequency. The rise time of the output $Q$ in the SF corner is $2.9 \mu \mathrm{s} \mathrm{s}$ and its fall time is 3.4 $\mu \mathrm{s}$, whereas they should be as close as possible to each other. In the FS corner, however, $D$ and $Q$ are clearly misbehaving.

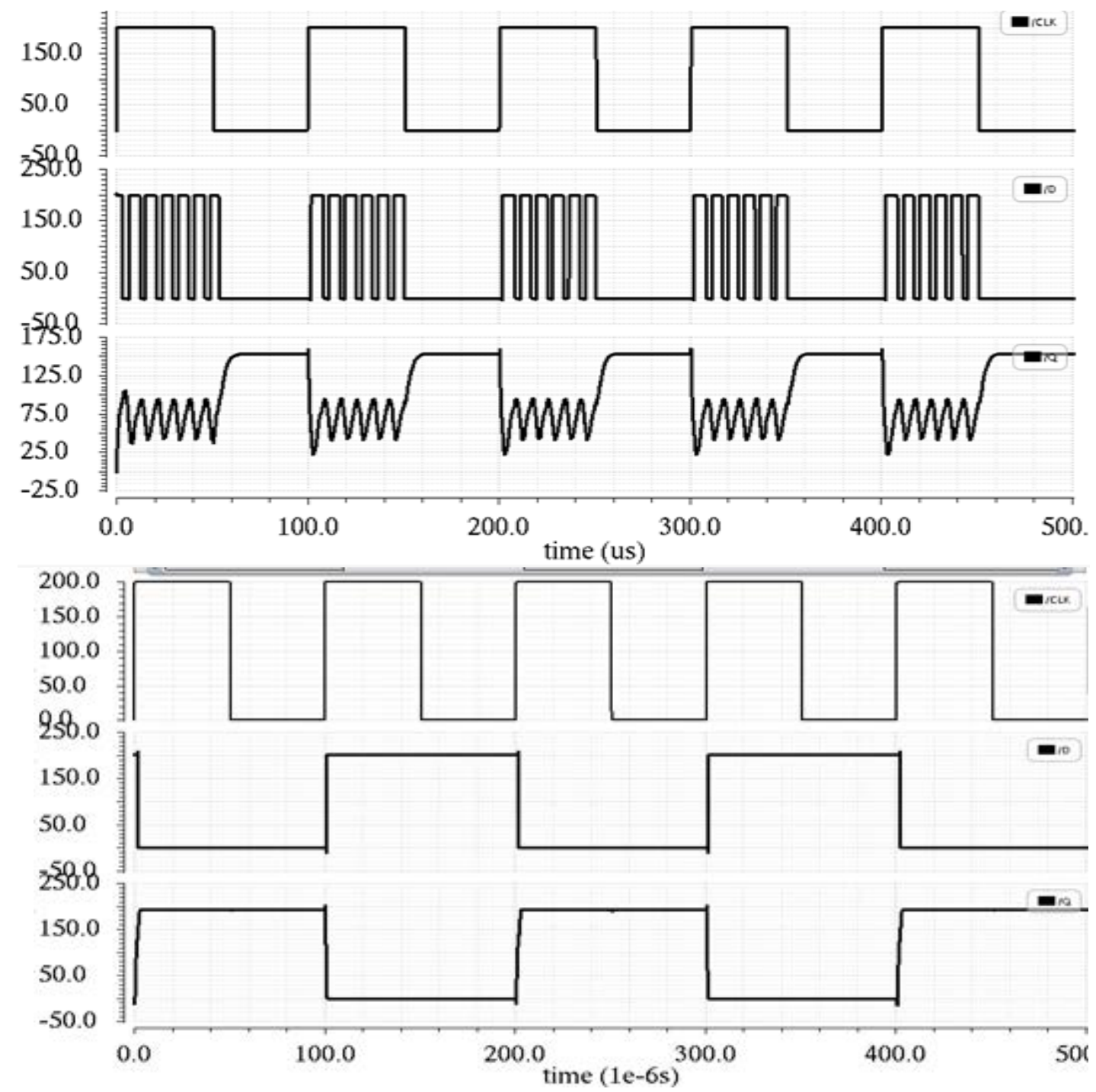

Figure 56 Transient response of minimum size TG flip-flop at FS and SF corner respectively $\left(V_{d d}=\right.$ $200 \mathrm{mV}, 28 \mathrm{~nm}$ process, svt). 
On the other hand, Monte Carlo simulations on 200 samples of the minimum-size TG-FF show that only 26 samples work properly, i.e. a yield of $13 \%$. Note that in the SF corner NMOS transistors are faster and the PMOS transistors are slower than their typical cases. The fact that the circuit's behaviour is worst in the FS corner, suggests that if we resize at least some of the critical NMOS and PMOS transistors in the flip-flop such that they match in speed as closely as possible, the yield could be improved.

Hence, we refer to Figure 45 and Figure 46 to find some small possible dimensions for NMOS and PMOS transistors with equal strength. These contour plots show that there are three such cases, as indicated on the figures by circles and small arrows:

1. $\mathrm{Wp} / \mathrm{Lp}=80 / 30$ and $\mathrm{Wn} / \mathrm{Ln}=80 / 70$ (smallest size case)

2. $\mathrm{Wp} / \mathrm{Lp}=300 / 30$ and $\mathrm{Wn} / \mathrm{Ln}=80 / 35$

3. $\mathrm{Wp} / \mathrm{Lp}=210 / 30$ and $\mathrm{Wn} / \mathrm{Ln}=80 / 40$

Table 11. The power, energy, and frequency of inverter chain $\left(V_{d d}=200 \mathrm{mV}, T=100 \mathrm{us}, 28 \mathrm{~nm}\right.$ process, svt, TT).

\begin{tabular}{|l|l|l|l|l|l|l|l|l|}
\hline & $\begin{array}{l}\boldsymbol{P}_{\text {stav }} \\
(\mathbf{p W})\end{array}$ & $\begin{array}{l}\boldsymbol{P}_{\boldsymbol{d}} \\
(\mathbf{p W})\end{array}$ & $\begin{array}{l}\boldsymbol{P}_{t} \\
(\mathbf{p W})\end{array}$ & $\begin{array}{l}\boldsymbol{E}_{\text {st }} \\
(\mathbf{f J})\end{array}$ & $\begin{array}{l}\boldsymbol{E}_{\boldsymbol{d}} \\
(\mathbf{f J})\end{array}$ & $\begin{array}{l}\boldsymbol{E}_{\boldsymbol{t}} \\
(\mathbf{f J})\end{array}$ & $\begin{array}{l}\boldsymbol{f}_{\max } \\
(\mathbf{M H Z})\end{array}$ & $\begin{array}{l}\boldsymbol{t}_{\boldsymbol{r}} / \boldsymbol{t}_{\boldsymbol{f}} \\
(\boldsymbol{\mu s})\end{array}$ \\
\hline $\begin{array}{l}\mathrm{Wp} / \mathrm{LP}=80 / 30 \\
\mathrm{Wn} / \mathrm{Ln}=80 / 70\end{array}$ & 3.25 & 36.35 & 39.6 & 0.004 & 0.056 & 0.06 & 0.65 & $1.1 / 0.8$ \\
\hline $\begin{array}{l}\mathrm{Wp} / \mathrm{LP}=300 / 30 \\
\mathrm{Wn} / \mathrm{Ln}=80 / 35\end{array}$ & 8.85 & 173.8 & 182.7 & 0.01 & 0.11 & 0.12 & 1.49 & $0.5 / 0.8$ \\
\hline $\begin{array}{l}\mathrm{Wp} / \mathrm{LP}=210 / 30 \\
\mathrm{Wn} / \mathrm{Ln}=80 / 40\end{array}$ & 6.16 & 84.44 & 90.6 & 0.007 & 0.091 & 0.097 & 0.92 & $0.6 / 0.2$ \\
\hline
\end{tabular}

We simulated a closed chain of five inverters with these sizes to compare their performances and power consumptions. The inverter chain is a suitable circuit for such basic evaluations because it does not need any external stimulus to oscillate. Table 11summarizes the results for the static and dynamic power and energy dissipations beside the frequency of operation. As expected, the smallest chain has the lowest static and dynamic energy dissipation, but also the lowest frequency of operation. The largest chain has the highest static and dynamic energy dissipations, but also the highest frequency of operations. The 
medium-size chain falls in between the two cases. Although increasing the length of transistors (above the feature length) is not a common practice in digital circuits design, we had to do that in the case of subthreshold circuits to obtain small equally strong PMOS and NMOS transistors. This is also advantageous for the reliability of circuits, as longer transistors have better process variations tolerance.

Referring back to the design of the TG-FF in Figure 55, beside the aforementioned case of using all minimum-size devices that resulted in a yield of $13 \%$, the following five transistor sizing cases are considered. We attempt to improve the yield case after case by only adjusting the dimensions of selected transistors to impose the least possible cost in terms of energy consumption. The default width and length of transistors are $W_{\min }=80 \mathrm{~nm}$ and $L_{\min }=30 \mathrm{~nm}$, unless otherwise mentioned.

- Sizing Case 1: LN3=LN4=LN5=LN6=70 $\mathrm{nm} \rightarrow$ yield $=21 \%$

(i.e. length of NMOS transistors in the feedback tri-state inverters)

Rationale: to lessen the strength of the feedback in fighting the forward-path transmission-gates.

- Sizing Case 2: LNT1 $=$ LNT2 $=$ LN3 $=$ LN4 $=$ LN5 $=L N 6=70 \mathrm{~nm} \rightarrow$ yield $=80 \%$ (i.e. length of NMOS transistors in the feedback tri-state inverters and TGs)

Rationale: to further improve the yield by matching the strength of PMOS and NMOS transistors in the TGs and reduce static power dissipation.

- Sizing Case 3: All $\mathrm{LN}=70 \mathrm{~nm} \rightarrow$ yield=100\%

Best Case

(i.e. length of all NMOS transistors, like $1^{\text {st }}$ inverter chain sizing)

Rationale: Matching the strength of all NMOS and PMOS transistors in flip-flop.

- Sizing Case 4: All LN=70 nm, except for LNT1=LNT2=LN3=LN4=LN5=LN6 $=40 \mathrm{~nm}$, and WPT1 $=W P T 2=210 \mathrm{~nm} \rightarrow$ yield $=100 \%$

(i.e. similar to $3^{\text {rd }}$ inverter chain sizing)

Rationale: Another option for equally strong PMOS and NMOS transistors in the circuit, while reducing length of feedback NMOS devices to compensate for wider TG PMOS devices.

- Sizing Case 5: All LN=70 nm, except for LNT1=LNT2=LN3=LN4=LN5=LN6 $=35 \mathrm{~nm}$, and WPT1 $=W P T 2=300 \mathrm{~nm} \rightarrow$ yield $=100 \%$

(i.e. similar to $2^{\text {nd }}$ inverter chain sizing) 
Rationale: Another option for equally strong PMOS and NMOS transistors in the circuit, while reducing length of feedback NMOS devices to compensate for wider TG PMOS devices.

Therefore, by choosing the three sizing options that equalizes the strength of the forward path NMOS and PMOS transistors in the TG-FF, we were able to reduce the failure rate of the circuit to $0 \%$ even when subjected to the most severe process variations. These results are illustrated in Figure 57. Among the three successful cases, Case 3 results in best static energy dissipations, because of having longest overall length of NMOS devices, and yet best dynamic power dissipation, because of having overall smallest PMOS devices. This case also produces almost matching rise and fall times at the output, as shown in Figure 58.

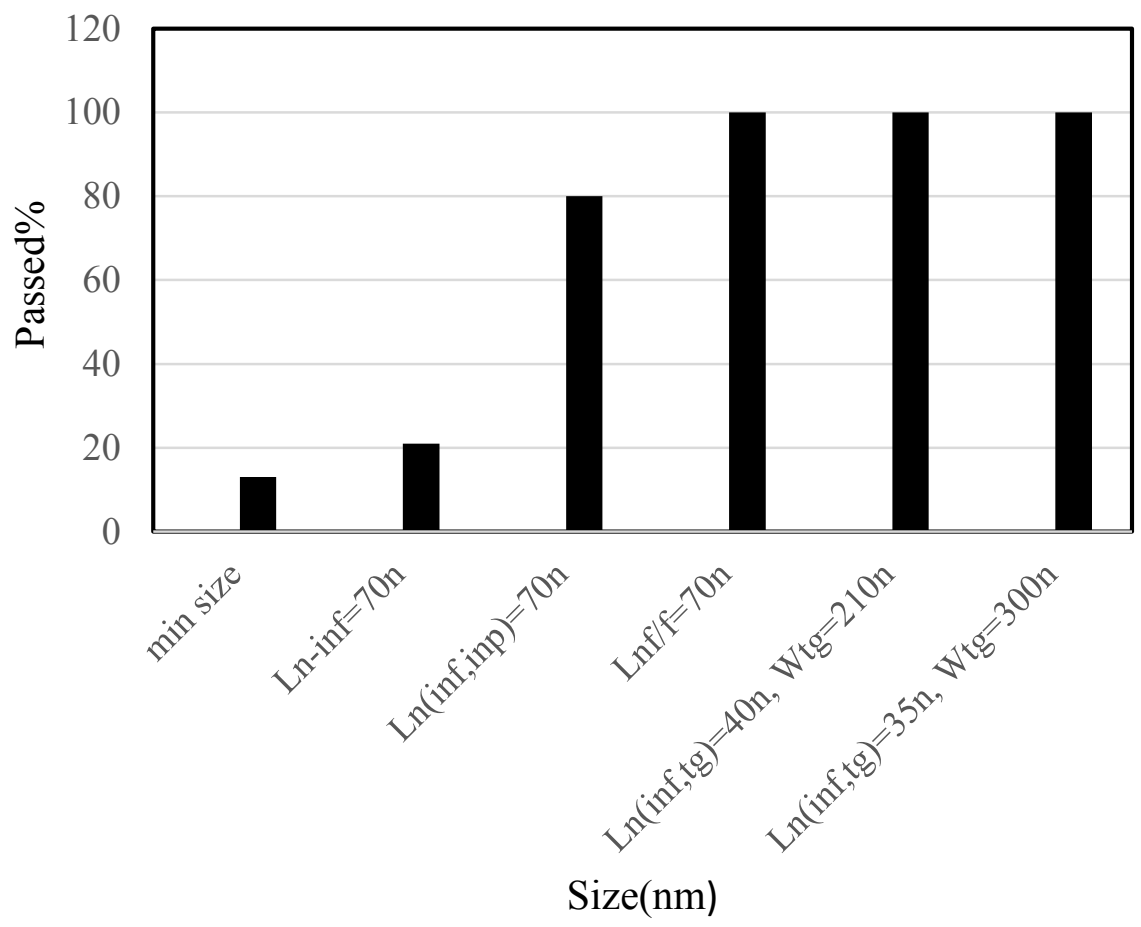

Figure 57 Number of passed of TG flip-flop for different sizes $\left(V_{d d}=200 \mathrm{mV}, 28 \mathrm{~nm}\right.$ process, svt). 


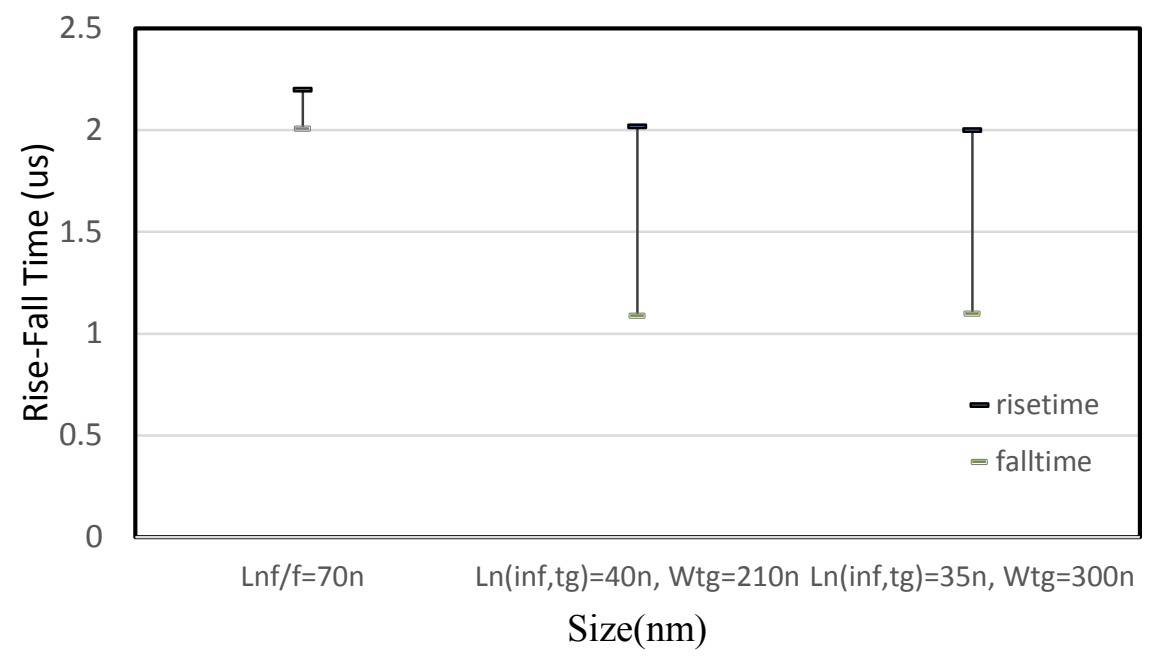

Figure 58 The rise and fall time of TG flip-flop output $\left(V_{d d}=200 \mathrm{mV}, 28 \mathrm{~nm}\right.$ process, svt).

\subsubsection{Single Phase Flip-Flop}

Figure 59 shows the schematic of the SP-FF. This circuit uses only one clock signal and is not prone to clock skew. Another advantage of this flip-flop is that it has two transistors less than the TG-FF. In the SP-FF circuit the TGs are replaced with NMOS and PMOS pass gates. Hence, the single-transistor pass gates have to pass $0 \mathrm{~s}$ and $1 \mathrm{~s}$. This particular issue makes the design of this flip-flop more challenging because of increased contentions and highly un-equal rising and falling transitions. 


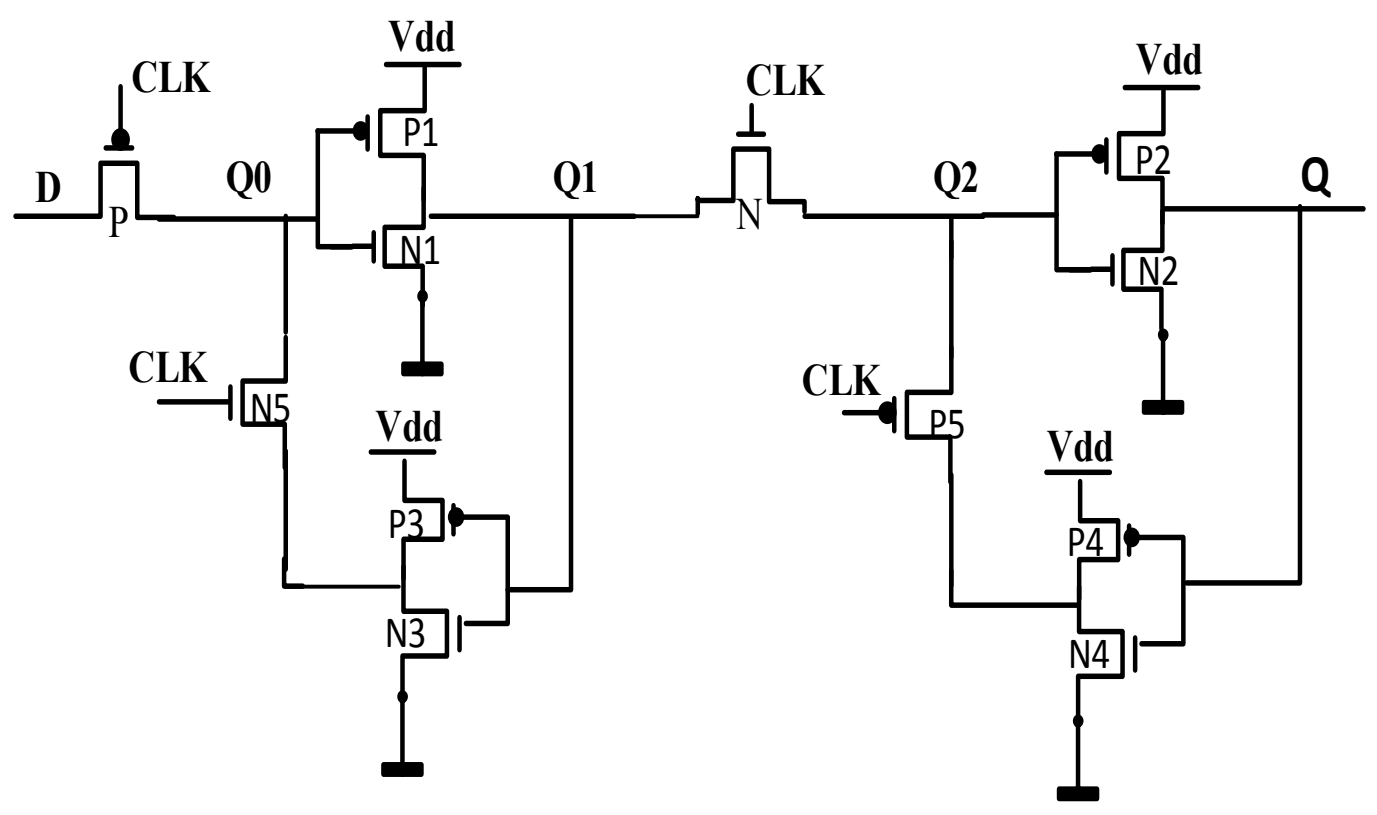

Figure 59 Single phase flip-flop schematic.

If built with all minimum-size transistors, the SP-FF fails to operate in all corners, except for the SF corner. Like in the case of the TG-FF, the worst behavior is observed in the FS corner, as illustrated in the timing diagrams of Figure 60. This again suggests that the failure is mainly due to the minimum-size NMOS transistor being much stronger than the minimum-size PMOS transistor. 


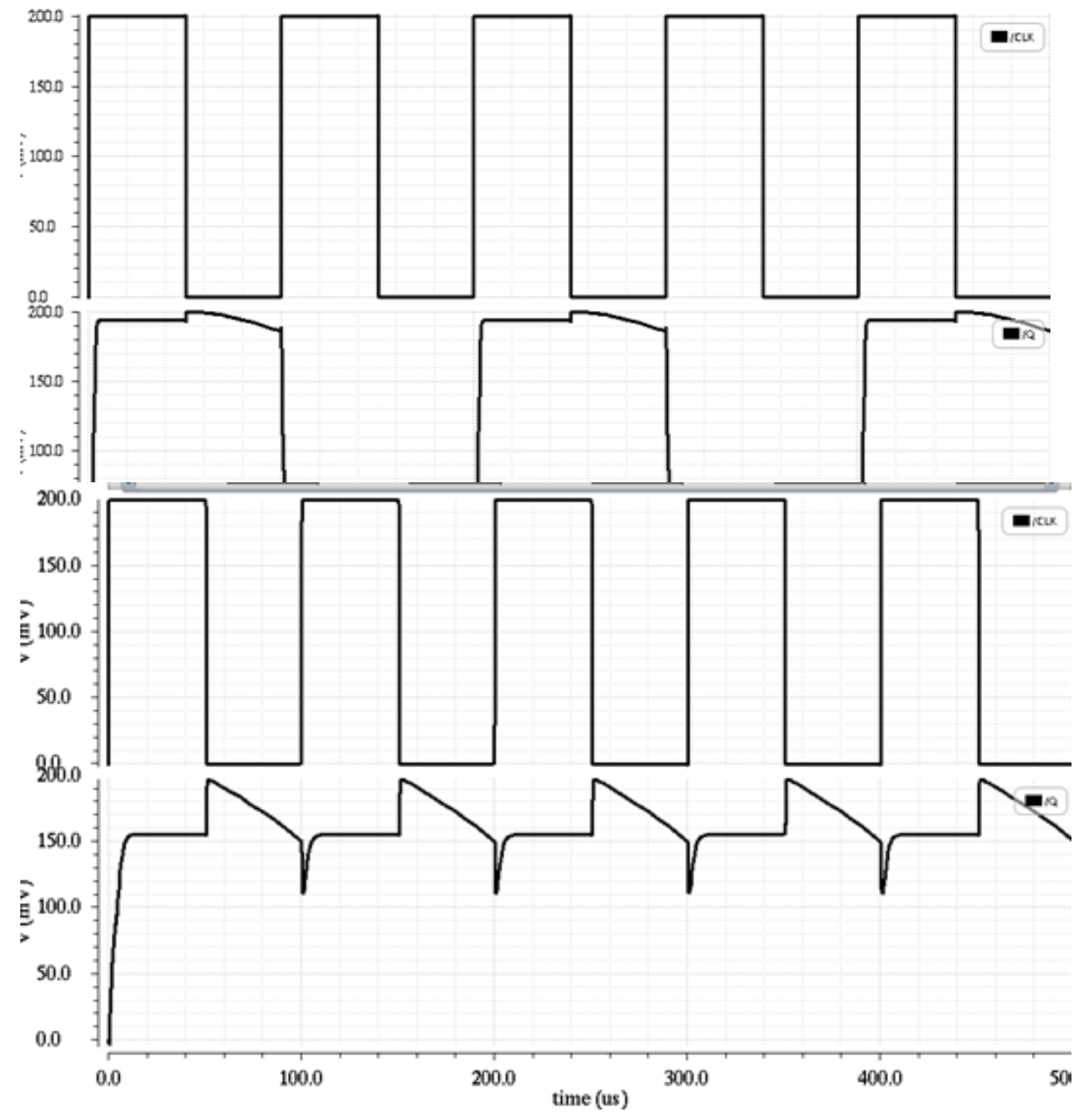

Figure 60 Transient response of TG flip-flop at SF and FS corner respectively ( $V_{d d}=200 \mathrm{mV}, 28 \mathrm{~nm}$ process, svt).

From the experience gained from the design of the TG-FF, we enlarge the length of NMOS transistors N, N2, and N5 to $70 \mathrm{~nm}$. As shown in Figure 61, the yield in this case is improved from only $8 \%$ to $78 \%$. If we further enlarge the length of all NMOS transistors in the circuit to $70 \mathrm{~nm}$ (Case 1), then the yield would be $100 \%$. The problem, however, in this case is that the falling transition at the output is much slower than the rising transition. 
Slow transitions, in turn, increase the short-circuit power dissipation of the following logic gates.

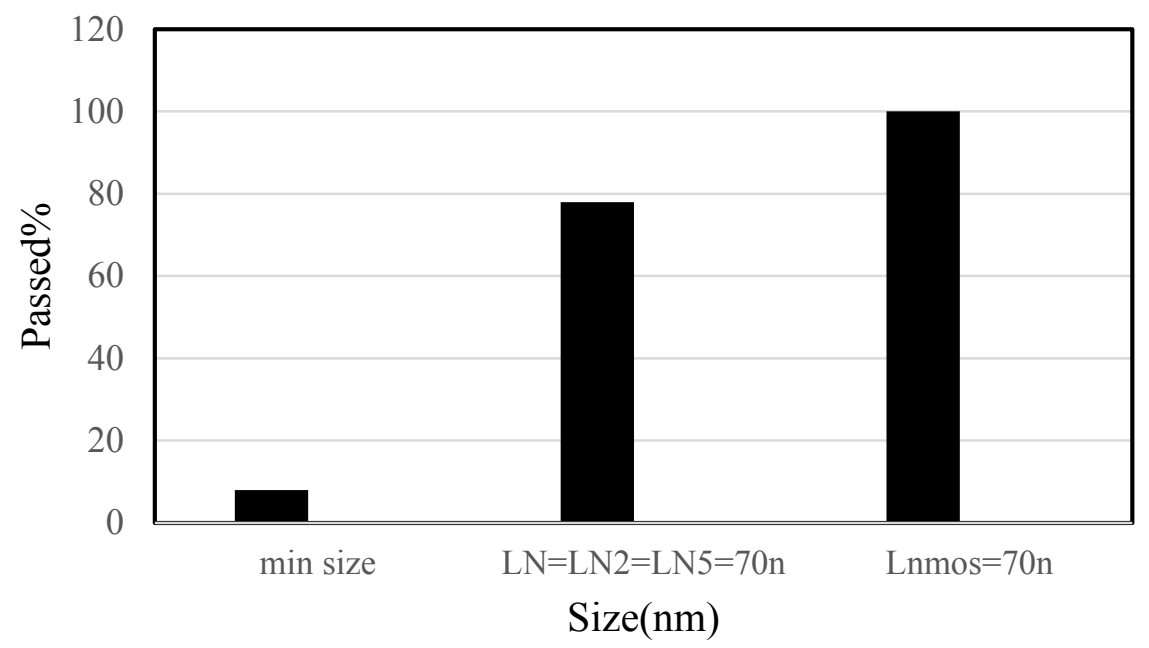

Figure 61 Number of passed of SP flip-flop ( $V_{d d}=200 \mathrm{mV}, 28 \mathrm{~nm}$ process, svt).

Table 12 shows a number of sizing attempts to bring the rise and fall times of the output closer to each other. First LN is reduced to $45 \mathrm{~nm}$ (from $70 \mathrm{~nm}$ ) to make the NMOS at the output stronger (Case 2). Although this helps, but still the fall time is twice the rise time. In the next attempt, we improve the strength of the pass gate $(\mathrm{N})$ driving the output inverter by shortening its length to $45 \mathrm{~nm}$ (Case 3). This makes the fall and rise times of the output fairly close. However, as shown in the timing diagram of Figure 62 (a), in this case the input PMOS transistor $\mathrm{P}$ is too slow in discharging node $Q 0$, which increases the short-circuit current of the inverter it drives. To remedy the problem, the width of $\mathrm{P}$ is enlarged to $300 \mathrm{~nm}$ (Case 4). As shown in Figure 62 (b) the problem at $Q 0$ is resolved while the output rise and fall times get even closer. Enlarging the PMOS pass gate naturally increases the static and dynamic energy consumptions. As alternative option, by referring to the contour plot in Figure 52, we replaced this larger P with a minimum-size lvt-type transistor (Case 5), which has the same strength but doesn't increase the energy consumptions as much. 
Table 12 The rising and falling of SP flip-flop output ( $V_{d d}=200 \mathrm{mV}, 28 \mathrm{~nm}$ process, svt).

\begin{tabular}{|l|l|l|c|}
\hline Transistor Sizing Cases for SP-FF & $\boldsymbol{t}_{\boldsymbol{r}}(\boldsymbol{\mu s})$ & $\boldsymbol{t}_{\boldsymbol{f}}(\boldsymbol{\mu} \mathbf{s})$ & Yield \% \\
\hline 1. Lnmos=70 nm & 2.12 & 15.83 & $100 \%$ \\
\hline 2. LN2=45 nm, Lnmos=70 nm & 2.17 & 5.70 & $100 \%$ \\
\hline 3. $\mathrm{LN}=\mathrm{LN} 2=45 \mathrm{~nm}, \mathrm{Lnmos}=70 \mathrm{~nm}$ & 2.08 & 2.19 & $100 \%$ \\
\hline 4. $\mathrm{LN}=\mathrm{LN} 2=45 \mathrm{~nm}, \mathrm{Lnmos}=70 \mathrm{n}, \mathrm{WP}=300 \mathrm{~nm}$ & 2.15 & 2.19 & $100 \%$ \\
\hline 5. $\mathrm{LN}=\mathrm{LN} 2=45 \mathrm{~nm}, \mathrm{Lnmos}=70 \mathrm{n}, \mathrm{P}$ is lvt-type & 2.15 & 2.19 & $100 \%$ \\
\hline
\end{tabular}

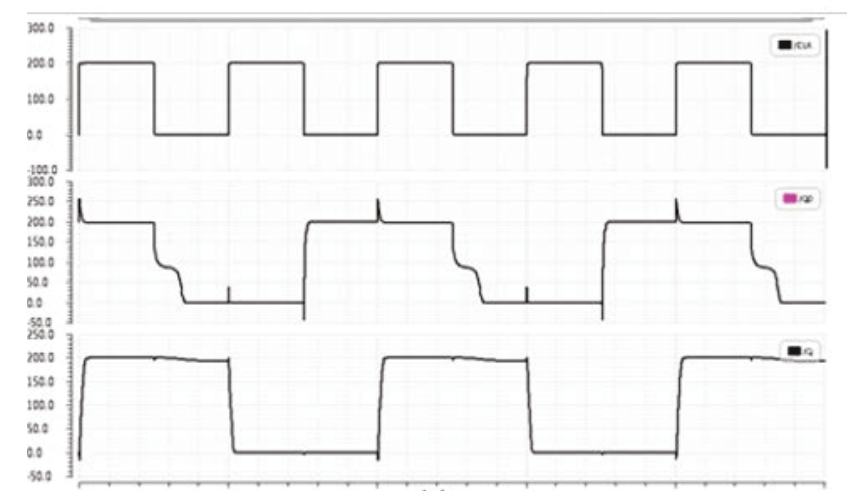

(a)
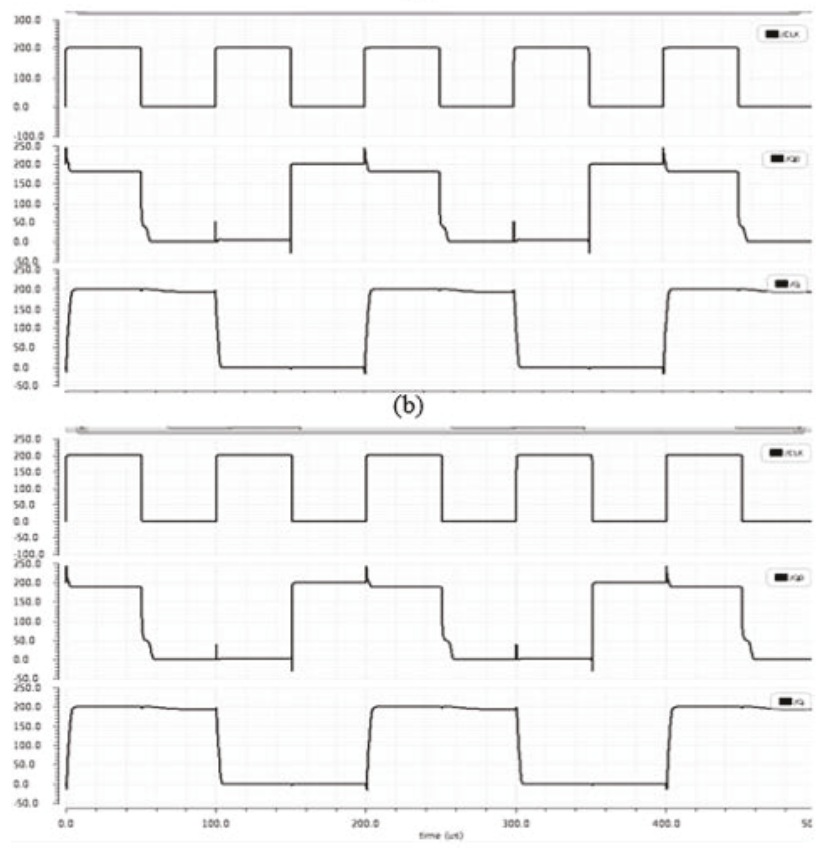

(c)

Figure 62 The output waveform $P$ of first latch of SP flip-flop (a) LN=LN2=45 nm (b) WP=300 $\mathrm{nm}$ (c) $P$ of lvt-type PMOS ( $V_{d d}=200 \mathrm{mV}, 28 \mathrm{~nm}$ process, svt). 
In the following sections we use the sizing Cases $4(\mathrm{Wp}=300 \mathrm{~nm})$ and $5(\mathrm{lvt}-\mathrm{P})$ for the SP-FF to compare with the TG-FF using its Case 3 sizing.

\subsection{Comparison between TG flip-flop and SP-flip-flop}

Figure 63 compares the three winning FF designs so far in terms of their power dissipations at various supply voltages. Both SP-FFs have comparable dynamic power dissipations that are about $30 \%$ lower than the power dissipation of the TG-FF at lower supply voltages. As the supply voltage increases the difference with the TG-FF shrinks, and the SP-FF with $300 \mathrm{~nm} W p$. The SP-FF with lvt-P shows superiority over the SP-FF with $300 \mathrm{~nm} W p$ in terms of the dynamic power dissipation, because of its lower capacitance, and also in terms of the static power dissipation, because of its lower leakage current (300 nm W svt-P versus minimum-size lvt-P). The TG-FF has a lower static power dissipation than both, because the SP-FFs have the more leaky transistors $\mathrm{P}$ and two NMOS transistors with $\mathrm{L}=45 \mathrm{~nm}$ (instead of all being $70 \mathrm{~nm}$ long). 

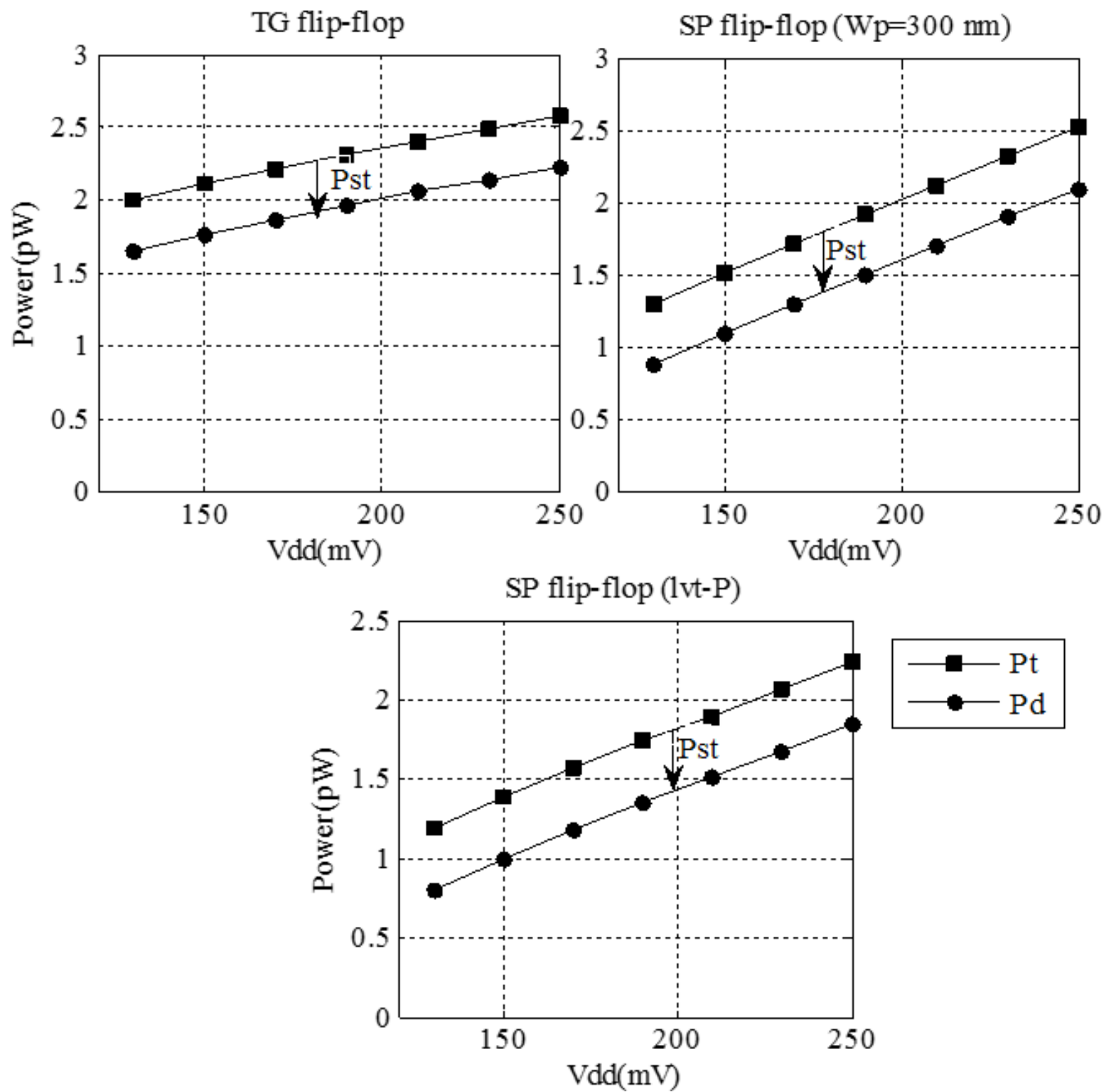

Figure 63 Total, static and dynamic power of TG, SP (WP =300nm), and SP (lvt-P) flip-flop $(T=100$ $\mu \mathrm{s}, 28 \mathrm{~nm}$ process).

Figure 64 compares the three flip-flops in terms their maximum frequency of operations. The three flip-flops require a clock period of at least 10 us (i.e. maximum frequency of $100 \mathrm{kHz}$ ) to operate properly. 

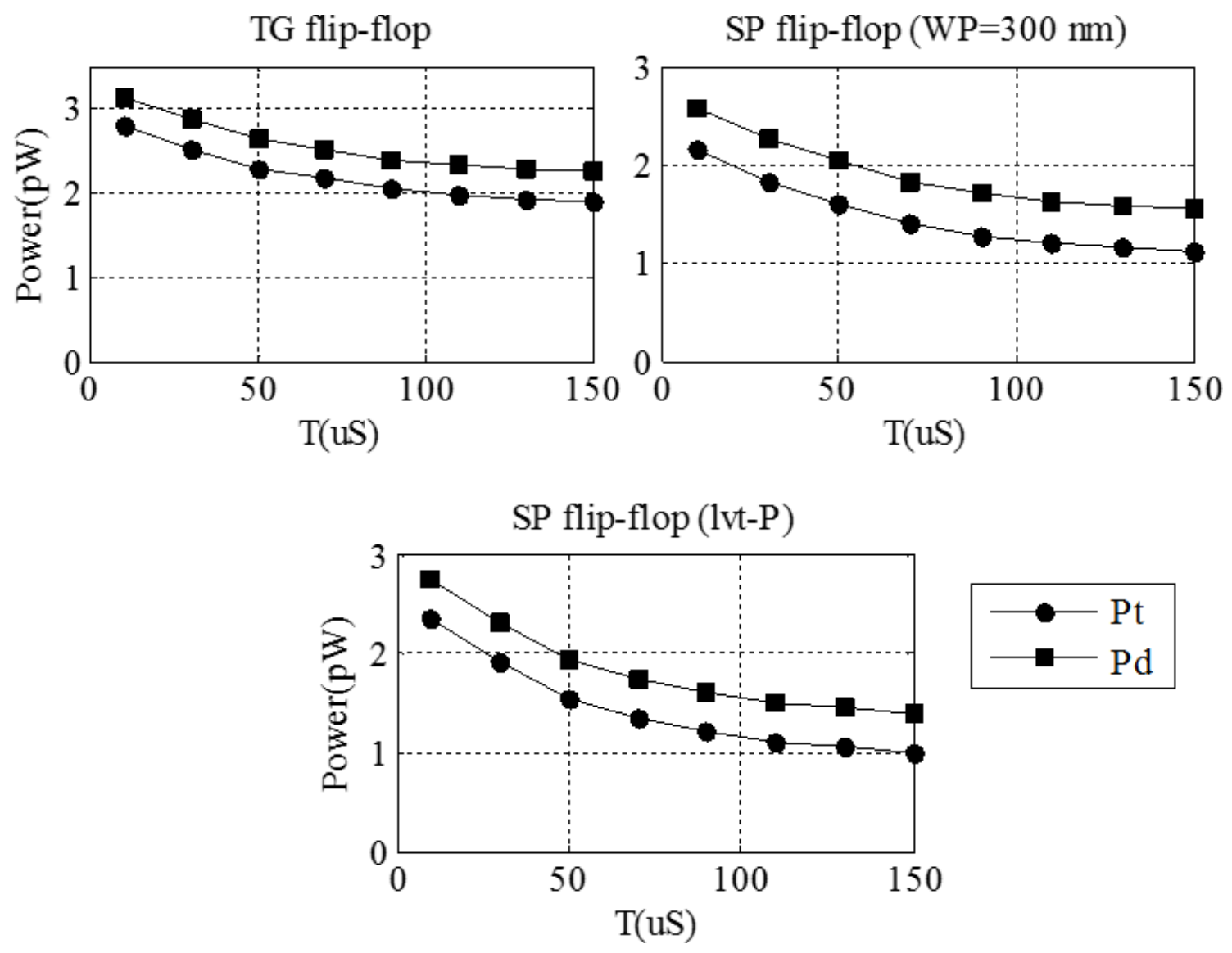

Figure $64 P_{t}, P_{d}, P_{s t}$ versus $T\left(V_{d d}=200 \mathrm{mV}, 28 \mathrm{~nm}\right.$ process $)$.

Figure 65 shows the rising and falling clock-to-output propagation delays for the three flip-flops versus the supply voltage. In the three flip-flops the rising and falling delays are fairly close over the range of $V_{d d}$. The TG-FF has higher delays because of its higher device count. However, it can operate with a minimum $V_{d d}$ of $110 \mathrm{mv}$ compared to $120 \mathrm{mv}$ in the cases of the SP flip-flops. 

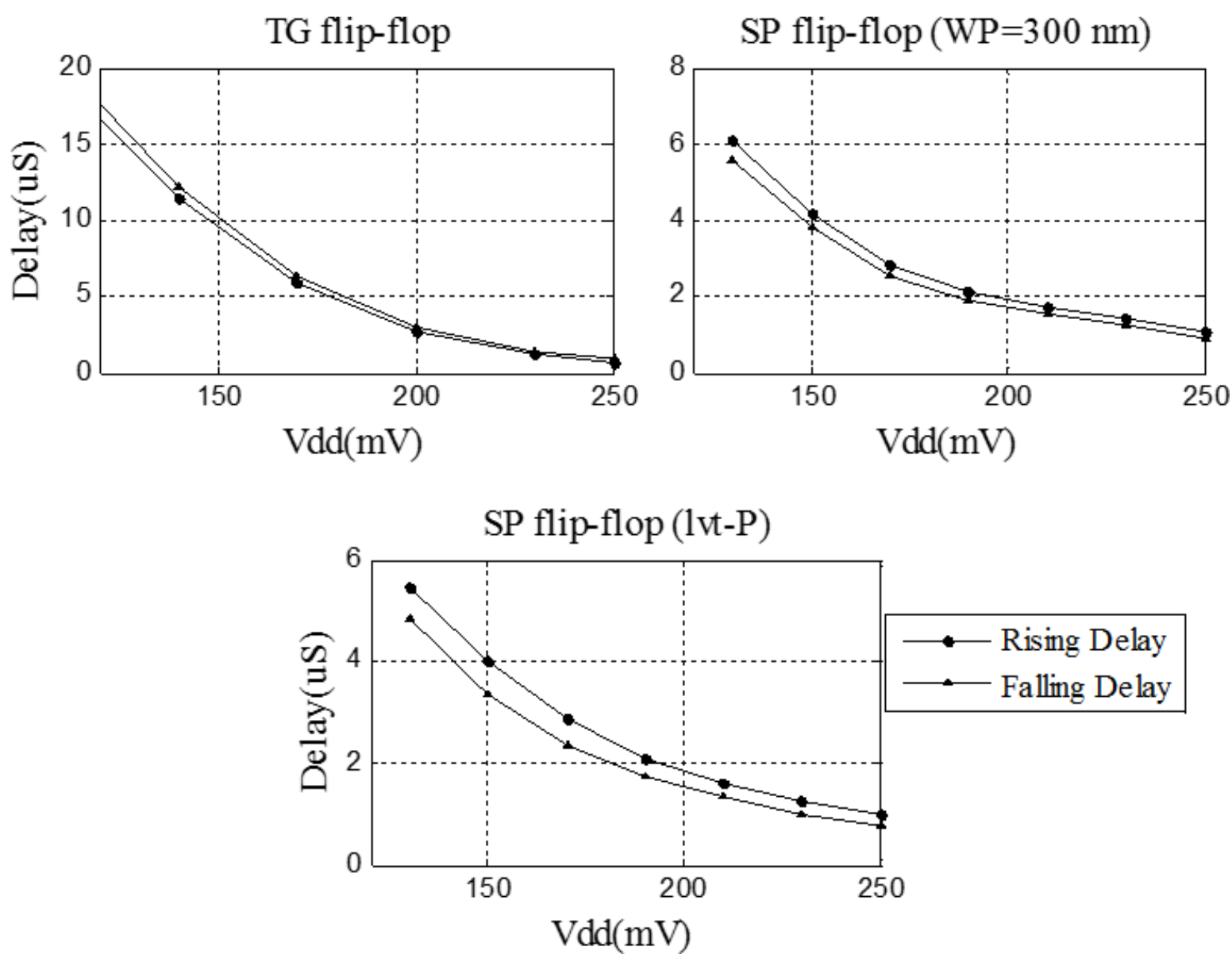

Figure $65 T_{c-q}$ versus $V_{d d}(T=100 \mu \mathrm{s}, 28 \mathrm{~nm}$ process). 

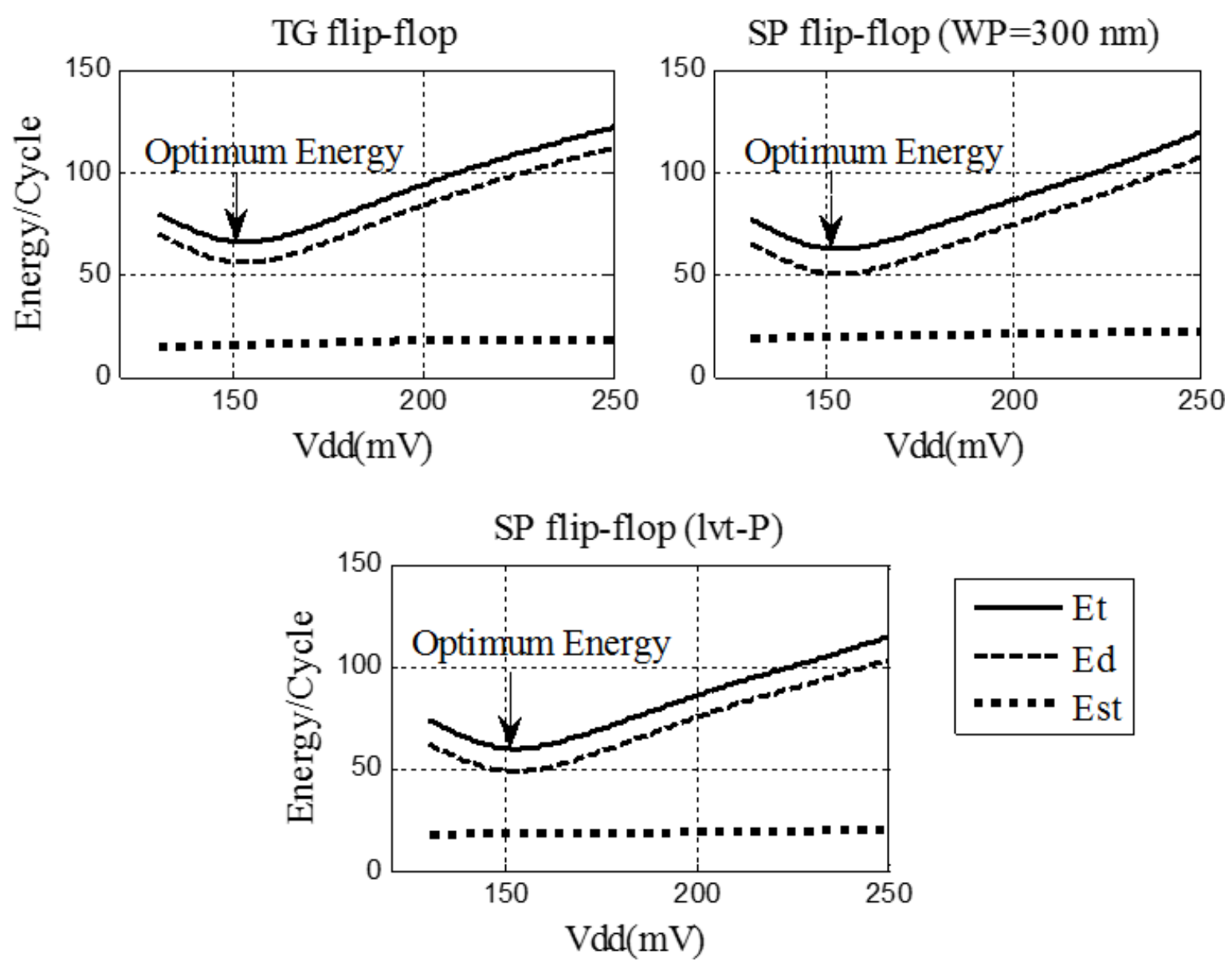

Figure 66 Energy per cycle versus supply voltage in $28 \mathrm{~nm}$ process.

Figure 66 shows the minimum-energy operation supply voltages for the three flipflops that are around $150 \mathrm{mV}$.

Finally, Table 13 shows a detailed comparison between the three flip-flops at $V_{d d}$ of $200 \mathrm{mv}$, while Table 14 presents the same comparison at the minimum-energy $V_{d d}$ of $150 \mathrm{mv}$. Based on these comparisons, we say that generally the SP flip-flops are close in features and superior to the TG flip-flop for subthreshold operation using the $28 \mathrm{~nm}$ technology. 
Table 13 Simulation results of flip-flops ( $V_{d d}=200 \mathrm{mV}, T=100 \mu \mathrm{s}, 28 \mathrm{~nm}$ process).

\begin{tabular}{|l|l|l|l|l|l|l|}
\hline Flip-Flop Configuration & $\boldsymbol{P}_{\text {stav }}$ & $\boldsymbol{P}_{\boldsymbol{d}}$ & $\boldsymbol{P}_{\text {total }}$ & $\boldsymbol{T}_{\boldsymbol{C} \text { - }}$ & $\boldsymbol{T}_{\text {setup }}$ & $\boldsymbol{f}_{\max }$ \\
\hline TG flip-flop & $0.35 \mathrm{p}$ & $2.01 \mathrm{p}$ & $2.36 \mathrm{p}$ & $2.96 \mu$ & $1.8 \mu$ & $105.17 \mathrm{k}$ \\
\hline $\begin{array}{l}\text { SP flip-flop } \\
\text { Ln=Ln2=45nm, } \\
\text { Ln5=30nm,Wp=300 nm) }\end{array}$ & $0.43 \mathrm{p}$ & $1.58 \mathrm{p}$ & $2.01 \mathrm{p}$ & $1.66 \mu$ & $1.49 \mu$ & $155.5 \mathrm{k}$ \\
\hline $\begin{array}{l}\text { SP flip-flop (lvt) } \\
\text { Ln=Ln2=45nm,Ln5=30nm } \\
\text { Transistor P is lvt-type }\end{array}$ & $0.39 \mathrm{p}$ & $1.42 \mathrm{p}$ & $1.81 \mathrm{p}$ & $1.67 \mu$ & $1.55 \mu$ & $125 \mathrm{k}$ \\
\hline
\end{tabular}

Table 14 The simulation results of flip-flop ( $V_{d d}=150 \mathrm{mV}, T=100 \mu \mathrm{s}, 28 \mathrm{~nm}$ process).

\begin{tabular}{|l|l|l|l|l|l|l|}
\hline Flip-Flop Configuration & $\boldsymbol{P}_{\boldsymbol{s}}$ & $\boldsymbol{P}_{\boldsymbol{d}}$ & $\boldsymbol{P}_{\text {total }}$ & $\boldsymbol{T}_{\boldsymbol{C}-\boldsymbol{Q}}$ & $\boldsymbol{T}_{\text {setup }}$ & $\boldsymbol{f}_{\max }$ \\
\hline $\begin{array}{l}\text { TG flip-flop } \\
\text { All Ln=70 nm }\end{array}$ & $0.33 \mathrm{p}$ & $1.9 \mathrm{p}$ & $2.13 \mathrm{p}$ & $9.98 \mu$ & $4.2 \mu$ & $47 \mathrm{k}$ \\
\hline $\begin{array}{l}\text { SP flip-flop } \\
\mathrm{Ln}=\mathrm{Ln} 2=45 \mathrm{~nm}, \mathrm{Ln} 5=30 \mathrm{~nm}, \\
\text { Wp=300 nm) }\end{array}$ & $0.42 \mathrm{p}$ & $1.09 \mathrm{p}$ & $1.51 \mathrm{p}$ & $4.11 \mu$ & $3.8 \mu$ & $52 \mathrm{k}$ \\
\hline $\begin{array}{l}\text { SP flip-flop (lvt) } \\
\text { Ln=Ln2=45 nm, Ln5=30 nm, } \\
\text { Transistor P is lvt-type }\end{array}$ & $0.38 \mathrm{p}$ & $1.03 \mathrm{p}$ & $1.41 \mathrm{p}$ & $4.11 \mu$ & $3.98 \mu$ & $51.5 \mathrm{k}$ \\
\hline
\end{tabular}

\subsection{Effect of Temperature and Supply Voltage Variations}

Table 15 shows a detailed comparison between the three flip-flops subject to temperature and $V_{d d}$ variations. The results are based on exhaustive Monte Carlo simulations at temperatures of $-40,27$, and $80^{\circ} \mathrm{C}$, and supply voltages of 180,200 , and $220 \mathrm{mV}$. As far as the TG flip-flop is concerned, its yield is $100 \%$ in all temperatures at $220 \mathrm{mv}$, but it reduces to $92 \%$ and $87 \%$ in $-40{ }^{\circ} \mathrm{C}$ at $200 \mathrm{mv}$ and $180 \mathrm{mv}$, respectively. Both SP flip-flops have a $100 \%$ yield, except in $-40^{\circ} \mathrm{C}$ at $180 \mathrm{mV}$ that is $98 \%$. The delay dispersion indicators (based on 
б) are almost the same for the three flip-flops in all cases. Thus, the three flip-flops are comparable in terms of sensitivity to PVT variations by ignoring the marginal superiority of the SP flip-flops. In terms of static and dynamic power dissipations, however, the SP flip-flops show superiority over the TG flip-flop, while the SP flip-flop with an lvt-P being an edge above the other one.

Table 15 Mean, Sigma, minimum, and maximum delay of TG and SP flip-flop (T=100 $\mu \mathrm{S}, 28 \mathrm{~nm}$ process).

\begin{tabular}{|c|c|c|c|c|}
\hline \multirow{21}{*}{$\begin{array}{c}\text { TG } \\
\text { FF } \\
\text { Delay }\end{array}$} & \multicolumn{4}{|c|}{$V_{d d}=220 \mathrm{~m}$} \\
\hline & & $\begin{array}{l}T=-40^{\circ} \mathrm{C} \\
\text { Yield }=100 \%\end{array}$ & $\begin{array}{l}T=27^{\circ} \mathrm{C} \\
\text { Yield }=100 \%\end{array}$ & $\begin{array}{l}T=80^{\circ} \mathrm{C} \\
\text { Yield }=100 \%\end{array}$ \\
\hline & Mean & $13.5 \mu \mathrm{s}$ & $1.85 \mu \mathrm{s}$ & $0.14 \mu \mathrm{s}$ \\
\hline & Sigma & $2.42 \mu \mathrm{s}$ & $0.27 \mu \mathrm{s}$ & $0.015 \mu \mathrm{s}$ \\
\hline & $\sigma$ & 0.18 & 0.14 & 0.1 \\
\hline & $D_{\min }$ & $8.89 \mu \mathrm{s}$ & $1.34 \mu \mathrm{s}$ & $0.11 \mu \mathrm{s}$ \\
\hline & $D_{\operatorname{Max}}$ & $24.03 \mu \mathrm{s}$ & $2.98 \mu \mathrm{s}$ & $0.19 \mu \mathrm{s}$ \\
\hline & \multicolumn{4}{|c|}{$V_{d d}=200 \mathrm{~m}$} \\
\hline & \multicolumn{2}{|r|}{$\begin{array}{l}T=-40{ }^{\circ} \mathrm{C} \\
\text { Yield }=92 \%\end{array}$} & $\begin{array}{l}T=27^{\circ} \mathrm{C} \\
\text { Yield }=100 \%\end{array}$ & $\begin{array}{l}T=80^{\circ} \mathrm{C} \\
\text { Yield }=100 \%\end{array}$ \\
\hline & Mean & $19.85 \mu \mathrm{s}$ & $3.054 \mu \mathrm{s}$ & $0.23 \mu \mathrm{s}$ \\
\hline & Sigma & $3.78 \mu \mathrm{s}$ & $0.46 \mu \mathrm{s}$ & $0.03 \mu \mathrm{s}$ \\
\hline & $\sigma$ & 0.19 & 0.15 & 0.12 \\
\hline & $D_{\min }$ & $12.8 \mu \mathrm{s}$ & $2.21 \mu \mathrm{s}$ & $0.17 \mu \mathrm{s}$ \\
\hline & $D_{\operatorname{Max}}$ & $36.3 \mu \mathrm{s}$ & $4.89 \mu \mathrm{s}$ & $0.3 \mu \mathrm{s}$ \\
\hline & \multicolumn{4}{|c|}{$V_{d d}=180 \mathrm{~m}$} \\
\hline & \multicolumn{2}{|r|}{$\begin{array}{l}T=-40^{\circ} \mathrm{C} \\
\text { Yield }=87 \%\end{array}$} & $\begin{array}{l}T=27^{\circ} \mathrm{C} \\
\text { Yield }=100 \%\end{array}$ & $\begin{array}{l}T=80^{\circ} \mathrm{C} \\
\text { Yield }=100 \%\end{array}$ \\
\hline & Mean & $24.66 \mu \mathrm{s}$ & $5.09 \mu \mathrm{s}$ & $0.37 \mu \mathrm{s}$ \\
\hline & Sigma & $4.98 \mu \mathrm{s}$ & $0.79 \mu \mathrm{s}$ & $0.048 \mu \mathrm{s}$ \\
\hline & $\sigma$ & 0.2 & 0.15 & 0.13 \\
\hline & $D_{\min }$ & $14.83 \mu \mathrm{s}$ & $3.59 \mu \mathrm{s}$ & $0.26 \mu \mathrm{s}$ \\
\hline & $D_{\operatorname{Max}}$ & $47.3 \mu \mathrm{s}$ & $8.04 \mu \mathrm{s}$ & $0.5 \mu \mathrm{s}$ \\
\hline \multirow{10}{*}{$\begin{array}{c}\text { SP } \\
\text { FF } \\
\text { Delay } \\
\left(\mathbf{W}_{\mathbf{P}}=\mathbf{3 0 0 n}\right)\end{array}$} & \multicolumn{4}{|c|}{$V_{d d}=220 \mathrm{~m}$} \\
\hline & \multicolumn{2}{|r|}{$\begin{array}{l}T=-40^{\circ} \mathrm{C} \\
\text { Yield }=100 \%\end{array}$} & $\begin{array}{l}T=27^{\circ} \mathrm{C} \\
\text { Yield }=100 \%\end{array}$ & $\begin{array}{l}T=80^{\circ} \mathrm{C} \\
\text { Yield }=100 \%\end{array}$ \\
\hline & Mean & $8.91 \mu \mathrm{s}$ & $1.21 \mu \mathrm{s}$ & $0.11 \mu \mathrm{s}$ \\
\hline & Sigma & $1.78 \mu \mathrm{s}$ & $0.18 \mu \mathrm{s}$ & $0.013 \mu \mathrm{s}$ \\
\hline & $\sigma$ & 0.19 & 0.14 & 0.11 \\
\hline & $D_{\min }$ & $5.16 \mu \mathrm{s}$ & $0.82 \mu \mathrm{s}$ & $0.08 \mu \mathrm{s}$ \\
\hline & $D_{\operatorname{Max}}$ & $16.12 \mu \mathrm{s}$ & $1.98 \mu \mathrm{s}$ & $0.15 \mu \mathrm{s}$ \\
\hline & \multicolumn{4}{|c|}{$V_{d d}=200 \mathrm{~m}$} \\
\hline & & $\begin{array}{l}T=-40^{\circ} \mathrm{C} \\
\text { Yield }=100 \%\end{array}$ & $\begin{array}{l}T=27^{\circ} \mathrm{C} \\
\text { Yield }=100 \%\end{array}$ & $\begin{array}{l}T=80^{\circ} \mathrm{C} \\
\text { Yield }=100 \%\end{array}$ \\
\hline & Mean & $13.31 \mu \mathrm{s}$ & $1.7 \mu \mathrm{s}$ & $0.13 \mu \mathrm{s}$ \\
\hline
\end{tabular}




\begin{tabular}{|c|c|c|c|c|}
\hline & Sigma & $2.58 \mu \mathrm{s}$ & $0.29 \mu \mathrm{s}$ & $0.014 \mu \mathrm{s}$ \\
\hline & $\sigma$ & 0.19 & 0.17 & 0.1 \\
\hline & $D_{\min }$ & $7.83 \mu \mathrm{s}$ & $1.15 \mu \mathrm{s}$ & $0.096 \mu \mathrm{s}$ \\
\hline & $D_{\operatorname{Max}}$ & $25.23 \mu \mathrm{s}$ & $2.9 \mu \mathrm{s}$ & $0.18 \mu \mathrm{s}$ \\
\hline & \multicolumn{4}{|c|}{$V_{d d}=180 \mathrm{~m}$} \\
\hline & \multicolumn{2}{|c|}{$\begin{array}{l}T=-40{ }^{\circ} \mathrm{C} \\
\text { Yield }=98 \%\end{array}$} & $\begin{array}{l}T=27^{\circ} \mathrm{C} \\
\text { Yield }=100 \%\end{array}$ & $\begin{array}{l}T=80^{\circ} \mathrm{C} \\
\text { Yield }=100 \%\end{array}$ \\
\hline & Mean & $19.51 \mu \mathrm{s}$ & $2.42 \mu \mathrm{s}$ & $0.15 \mu \mathrm{s}$ \\
\hline & Sigma & $4.01 \mu \mathrm{s}$ & $0.46 \mu \mathrm{s}$ & $0.017 \mu \mathrm{s}$ \\
\hline & $\sigma$ & 0.2 & 0.19 & 0.11 \\
\hline & $D_{\min }$ & $10.07 \mu \mathrm{s}$ & $1.55 \mu \mathrm{s}$ & $0.11 \mu \mathrm{s}$ \\
\hline & $D_{\operatorname{Max}}$ & $38.77 \mu \mathrm{s}$ & $4.26 \mu \mathrm{s}$ & $0.22 \mu \mathrm{s}$ \\
\hline \multirow{21}{*}{$\begin{array}{c}\text { SP } \\
\text { FF } \\
\text { Delay } \\
\text { (lvt) }\end{array}$} & \multicolumn{4}{|c|}{$V_{d d}=220 \mathrm{~m}$} \\
\hline & \multicolumn{2}{|r|}{$\begin{array}{l}T=-40{ }^{\circ} \mathrm{C} \\
\text { Yield }=100 \%\end{array}$} & $\begin{array}{l}T=27^{\circ} \mathrm{C} \\
\text { Yield }=100 \%\end{array}$ & $\begin{array}{l}T=80{ }^{\circ} \mathrm{C} \\
\text { Yield }=100 \%\end{array}$ \\
\hline & Mean & $8.88 \mu \mathrm{s}$ & $1.21 \mu \mathrm{s}$ & $0.11 \mu \mathrm{s}$ \\
\hline & Sigma & $1.69 \mu \mathrm{s}$ & $0.17 \mu \mathrm{s}$ & $0.012 \mu \mathrm{s}$ \\
\hline & $\sigma$ & 0.19 & 0.14 & 0.1 \\
\hline & $D_{\min }$ & $5.11 \mu \mathrm{s}$ & $0.81 \mu \mathrm{s}$ & $0.075 \mu \mathrm{s}$ \\
\hline & $D_{\operatorname{Max}}$ & $16.06 \mu \mathrm{s}$ & $2.05 \mu \mathrm{s}$ & $0.14 \mu \mathrm{s}$ \\
\hline & \multicolumn{4}{|c|}{$V_{d d}=200 \mathrm{~m}$} \\
\hline & & $\begin{array}{l}T=-40{ }^{\circ} \mathrm{C} \\
\text { Yield }=100 \%\end{array}$ & $\begin{array}{l}T=27^{\circ} \mathrm{C} \\
\text { Yield }=100 \%\end{array}$ & $\begin{array}{l}T=80{ }^{\circ} \mathrm{C} \\
\text { Yield }=100 \%\end{array}$ \\
\hline & Mean & $13.48 \mu \mathrm{s}$ & $1.71 \mu \mathrm{s}$ & $0.12 \mu \mathrm{s}$ \\
\hline & Sigma & $2.48 \mu \mathrm{s}$ & $0.3 \mu \mathrm{s}$ & $0.013 \mu \mathrm{s}$ \\
\hline & $\sigma$ & 0.18 & 0.17 & 0.1 \\
\hline & $D_{\min }$ & $7.81 \mu \mathrm{s}$ & $1.1 \mu \mathrm{s}$ & $0.09 \mu \mathrm{s}$ \\
\hline & $D_{\operatorname{Max}}$ & $27.77 \mu \mathrm{s}$ & $2.97 \mu \mathrm{s}$ & $0.17 \mu \mathrm{s}$ \\
\hline & \multicolumn{4}{|c|}{$V_{d d}=180 \mathrm{~m}$} \\
\hline & & $\begin{array}{l}T=-40{ }^{\circ} \mathrm{C} \\
\text { Yield }=98 \%\end{array}$ & $\begin{array}{l}T=27{ }^{\circ} \mathrm{C} \\
\text { Yield }=100 \%\end{array}$ & $\begin{array}{l}T=80^{\circ} \mathrm{C} \\
\text { Yield }=100 \%\end{array}$ \\
\hline & Mean & $19.71 \mu \mathrm{s}$ & $2.44 \mu \mathrm{s}$ & $0.15 \mu \mathrm{s}$ \\
\hline & Sigma & $4.1 \mu \mathrm{s}$ & $0.47 \mu \mathrm{s}$ & $0.017 \mu \mathrm{s}$ \\
\hline & $\sigma$ & 0.2 & 0.19 & 0.11 \\
\hline & $D_{\min }$ & $10.03 \mu \mathrm{s}$ & $1.51 \mu \mathrm{s}$ & $0.11 \mu \mathrm{s}$ \\
\hline & $D_{\operatorname{Max}}$ & $38.9 \mu \mathrm{s}$ & $4.33 \mu \mathrm{s}$ & $0.22 \mu \mathrm{s}$ \\
\hline
\end{tabular}




\subsection{Summary}

In this chapter, we presented the details of a new design methodology and applied it to improve the reliability of flip-flop circuits operating in the subthreshold region. We also reported detailed comparisons between the proposed flip-flop designs in terms of power and energy consumptions, delay, minimum operational supply voltage, minimum-energy supply voltage, and tolerance towards PVT variations. Conclusions and suggestions for future work are discussed in the next chapter. 


\section{Chapter 8. Conclusions}

Ultra-low-power integrated circuits take advantage of operating at the subthreshold region. Subthreshold circuits, however, suffer from very low tolerance towards process, supply voltage, and temperature (PVT) variations. The task of designing reliable subthreshold circuits becomes further challenging in the case flip-flops that have inherent timing constraints. Subthreshold flip-flops cannot be simply designed with the commonly-used minimum-size devices only that are usually best suited for minimizing energy dissipation.

This thesis addresses the challenge of designing reliable and energy-efficient subthreshold flip-flops. A 16-transistor dual-clock-phase flip-flop (TG-FF) and a 12-transistor single-clock-phase flip-flop (SP-FF) configurations are considered and their gradual design modifications are reported towards perfecting their yields at minimum cost in terms of energy consumption. In addition, the two optimized flip-flop designs are compared in details based on the criteria of static and dynamic energy consumptions, delay, and reliability in face of PVT variations. For the purpose of accurate evaluations, we designed a test bench and developed methods for measuring the metrics.

A $65 \mathrm{~nm}$ bulk CMOS technology kit and a newer $28 \mathrm{~nm}$ FD-SOI CMOS technology kit were used during the course of this work. In both cases, the work started by device characterization to understand the behaviour of the subthreshold current as the device dimensions change. In the $65 \mathrm{~nm}$ technology this was done using 2D graphs, while in the 28 $\mathrm{nm}$ technology we improved the technique by using more convenient and more informative $3 \mathrm{D}$ contour graphs. Furthermore, in the $65 \mathrm{~nm}$ technology multi-threshold MOSFETs were incorporated for yield optimization, while in the $28 \mathrm{~nm}$ technology ultimately a combination transistor sizing and multi-threshold incorporation was used.

In the $65 \mathrm{~nm}$ technology we were able improve the reliability of the TG-FF from $29 \%$ in the room temperature at the nominal $V_{d d}$ of $200 \mathrm{mv}$ to $100 \%$ in the same condition, and to $75 \%$ in the extreme cold at $180 \mathrm{mV}$. The reliability of the SP-FF was also improved from $17 \%$ in the room temperature at nominal condition to $100 \%$, and to $87 \%$ in the extreme cold at $180 \mathrm{mV}$. In the $28 \mathrm{~nm}$ technology the reliability of the TG-FF was improved 
from $13 \%$ at the nominal condition to $100 \%$, and to $87 \%$ in the extreme cold at $180 \mathrm{mV}$. The reliability of the SP-FF was also improved from $8 \%$ at the nominal condition to $100 \%$, and to $98 \%$ in the extreme cold at $180 \mathrm{mV}$.

Comparing the TG-FF to the SP-FF, we may say that overall the SP-FF can be designed to be superior for subthreshold operation in terms of static and dynamic energy dissipations, delay, and tolerance with respect to PVT variations.

\subsection{Thesis Contributions}

1. Characterization of the NMOS/PMOS low- $V_{t h} /$ standard- $V_{t h} /$ high- $V_{t h}$ devices in the $65 \mathrm{~nm}$ bulk CMOS technology through analyzing the effect of manipulating the channel width and length of on the ON (operating) current, OFF (idle) current, and threshold voltage

2. Introducing an innovative and convenient method of device characterization through the use of contour graphs to analyse the behaviour of the operating and idle current, threshold voltage, and dispersion of these parameters as the channel length and width change

3. Characterization of the NMOS/PMOS low- $V_{t h} /$ standard- $V_{t h}$ in the $28 \mathrm{~nm}$ FDSOI CMOS technology by the use of the contour graphs mentioned above (as the first Carleton user of this most advanced IC technology kit available to $\mathrm{Ca}-$ nadian academia)

4. Introducing a test bench and measurement techniques for a fair comparison between flip-flop circuits and evaluating the static and dynamic power and energy consumptions beside the delay and timing figures of flip-flop circuits

5. Performing reliability analysis of the basic circuit components, including inverter, transmission gate, and tri-state buffer

6. Introducing an innovative method to automatically determine the failure of a flip-flop circuit operation

7. Use of exhaustive Monte Carlo simulations to evaluate the reliability of flipflop circuits subjected to PVT variations 
8. Incorporating multi-threshold MOS devices to improve the reliability of a common dual-clock-phase flip-flop (TG-FF) and a single-phase flip-flop (SP-FF) for subthreshold operation, with minimal cost in energy

9. Using transistor-sizing technique to improve the reliability of the TG-FF and SO-FF for subthreshold operation, with minimal cost in energy

10. Combining transistor-sizing technique and incorporation of multi-threshold devices (in carefully selected positions) to improve the reliability of the TG-FF and SP-FF for subthreshold operation, with minimal cost in energy

11. Enhancing the reliability of the TG-FF and SP-FF, operating under nominal temperature and supply voltage condition $\left(27^{\circ} \mathrm{C}\right.$ and $\left.200 \mathrm{mV}\right)$, from a combined average of $23 \%$ to $100 \%$ in the $65 \mathrm{~nm}$ technology and from a combined average of $11 \%$ to 100 in the $28 \mathrm{~nm}$ technology

12. Enhancing the reliability of the TG-FF and SP-FF, operating under adverse temperature and supply voltage condition $\left(-40{ }^{\circ} \mathrm{C}\right.$ and $\left.180 \mathrm{mV}\right)$, to a combined average of $81 \%$ in the $65 \mathrm{~nm}$ technology and a combined average of $93 \%$ in the $28 \mathrm{~nm}$ technology

13. Performing a detailed comparison between the TG-FF and SP-FF as optimized for reliability based on the lowest operational supply voltage, minimum-energy supply voltage, and maximum frequency of operation

14. Recommending the SP-FF as the better choice overall for subthreshold operation (in contrast to superthreshold operation)

\subsection{Future work}

Some suggestions for future work are as follows.

1. Implementing the layouts of the optimized flip-flops (preferably in the $28 \mathrm{~nm}$ ) technology and evaluating the metrics based on extracted layout to include

2. Performing Monte Carlo simulations based on the extracted layout

3. Fabrication and testing of the flip-flops and testing the dies under nominal and adverse temperature and supply voltage conditions, and evaluating the yields. 
4. Design, fabrication, and test of subthreshold circuit applications for the flipflops such as shift registers, counters, and FSMs

5. Extending the work to the design of other flip-flop topologies for subthreshold operation

6. Extending the work to the design of reliable subthreshold memory cells 


\section{References}

[1] A. Wang, A. P. Chandraksan, and S. V. Kosonocky, "Optimal supply and threshold scaling for subthreshold CMOS circuits," in Proceedings of IEEE Computer Society Annual Symposium on VLSI, Pittsburgh, 2002.

[2] J. Burr and A. Peterson, "Ultra low power CMOS technology," in Proceeding of 3rd NASA Symposium on VLSI Design, 1991.

[3] A. Wang, B. Calhoun, and A. chandrakasan, Sub-threshold Design for Ultra Lowpower Systems, Newyork: Springer, 2006.

[4] B. H. Calhoun and A. Chandrakasan, "Characterizing and modeling minimum energy operation for subthreshold circuits," in Proc. of IEEE International Symposium on Low Power Electronics and Design Conference (ISPLED'04), Newport Beach, CA, Aug. 2004.

[5] F. Leuenberger and E. Vittoz, "Complementary-MOS low-power low-voltage integrated binary counter," Proceedings of the IEEE , vol. 57, no. 9, pp. 1528-1532, Sept. 1969.

[6] R. M. Swanson and J. D. Meindl, "Ion-implanted complementary MOS transistors in low-voltage circuits," IEEE Journal of Solid-State Circuits, vol. 7, no. 2, pp. 146153, Apr. 1972.

[7] A. Wang and A. Chandrakasan, "A $180 \mathrm{mV}$ subthreshold FFT processor using a minimum energy design methodology," IEEE Journal of Solid-State Circuits, vol. 40, no. 1, pp. 310-319, Jan. 2005.

[8] B. H. Calhoun, A.Wang, and A. Chandrakasan, "Modeling and sizing for minimum energy operation in subthreshold circuits," IEEE Journal of Solid-State Circuits, vol. 40, no. 9, pp. 1778-1786, Sep. 2005.

[9] J. Chen, L. T. Clark, and T.-H. Chen, "An ultra-low-power memory with a subthreshold power supply voltage," IEEE Journal of Solid-State Circuits, vol. 41, no. 10, pp. 2344-2353, Oct. 2006.

[10] A. Argarwal, N. Banerjee, S. K. Hsu, R. K. Krishnamurthy, and K. Roy, "A 200mV to $1.2 \mathrm{~V}, 4.4 \mathrm{MHz}$ to $6.3 \mathrm{GHz}, 48 \mathrm{x} 42 \mathrm{~b} 1 \mathrm{R} / 1 \mathrm{~W}$ programmable register file in $65 \mathrm{~nm}$ CMOS," in IEEE International conference on Solid State Circuits, Munich, 2007.

[11] S. Hanson, B. Zhai, M. Seok, B. Cline, K. Zhou, M. Singhal, M. Minuth, J. Olson, L. Nazhandali, T. Austin, D. Sylvester, and D. Blaauw, "Exploring variability and performance in a sub-200-mV processor," IEEE Journal of Solid-State Circuits, vol. 43, no. 4, p. 881-891, Apr. 2008.

[12] MattmannMoritz , Bechstein, Daniel , C. Roman, Chikkadi, Kiran, Hierold, Christofer, "Reduction of gate hysteresis above ambient temperature via ambipolar pulsed gate sweeps in carbon nanotube field effect transistors for sensor applications," Applied Physics Letters, vol. 97, no. 15, pp. 153103-153103-3, 2010. 
[13] H. Soeleman, K. Roy, and B.C. Paul, "Robust subthreshold logic for ultra-low power," IEEE Transactions on Very Large Scale Integration (VLSI) Systems, vol. 9, no. 1, pp. 90-99, 2001.

[14] A. Tajalli, M. Alioto and Y. Leblebici, "Improving Power-Delay Performance of Ultr-Low Power Subthreshold SCL Circuits," IEEE Transactions on Circuits and Systems II: Express Briefs, vol. 56, no. 2, pp. 127-131, 2009.

[15] M. Muker and M. Shams, "Designing digital subthreshold CMOS circuits using parallel transistor stacks," Electronic Letters, vol. 47, no. 6, pp. 372-374, 2011.

[16] A. Hasanbegovic and S. Aunet, "Low-power subthreshold to above threshold level shifter in $90 \mathrm{~nm}$ process," in Norchip, Trondheim, 2009.

[17] Bo Fu and P. Ampadu, "Comparative analysis of ultra-low voltage flip-flops for energy efficiency," in IEEE International Symposium on Circuits and Systems. ISCAS 2007, New Orleans, LA, 2007.

[18] H.P. Alstad and S. Aunet, "Seven subthreshold flip-flop cells," in Norchip, Aalborg, 2007.

[19] J. M. Rabaey, A. Chandrakasan, and B. Nikolic, Digital Integrated Circuits A Design Perspective, New jersey: Prentice Hall, Upper Saddle River, 2003.

[20] A. Chavan, G. Dukle, B. Graniello, and E. MacDonald, "Robust ultra-low power subthreshold logic flip-flop design for reconfigurable architectures," in IEEE International Conference on Reconfigurable Computing and FPGA's, 2006. ReConFig 2006, San Luis Potosi, 2006.

[21] N. H. E. Weste and D. M. Harris, CMOS VLSI Design, A Circuits and Systems Perspective, Toronto: Addison-Wesley, 2011.

[22] A. Morgenstein, A. Fish, I. Wagner, "An Efficient Implementation of D-Flip-Flop Using the GDI Technique," in Proceedings of the 2004 International Symposium on Circuits and Systems, ISCAS 04., May 2004.

[23] Sagi Fisher, Adam Teman, Dmitry Vaysman, Alexander Gertsman,Orly YadidPecht, "Ultra-Low Power Subthreshold Flip-Flop Design," in IEEE International Symposium on Circuits and Systems, ISCAS 2009. , Taipei, 2009.

[24] Neelam Swami, Neha Arora, Prof. B.P.Singh, "Low Power Subthreshold D FlipFlop," in International Conference onvDevices and Communications (ICDeCom), Mesra, 2011.

[25] A. Chatterjee, M. Nandakumar, Ih-Chin Chen , "An Investigation of the Impact of Technology Scaling on Power Wasted as Short-circuit Current in Low Voltage Static CMOS Circuits," in International Symposium on Low Power Electronics and Design, Monterey, CA, 1996.

[26] B. Razavi, Design of Analog CMOS Integrated Circuits, Boston: Mc Graw Hill, 2001.

[27] K. Roy, S. Mukhopadhhyay and H. Mahmoodi-Meimand, "Leakage Current Mechanisms and Leakage Reduction Techniques in Deep-Submicrometer CMOS Circuits," Proceedings of The IEEE, vol. 91, no. 2, pp. 305-327, Feb. 2003.

[28] S. Borkar, "Low power design challenges for the decade," in Proceedings of the ASPDAC 2001. on Design Automation, Yokohama, January 2001. 
[29] J. Kao, S. Narendra, and A. Chandrakasan, "Subthreshold leakage modeling and reduction techniques," in IEEE/ACM International Conference on Computer Aided Design, ICCAD 2002. , November 2002.

[30] J. B. Kuo and J. H. Lou, Low-Voltage CMOS VLSI Circuits, Newyork: Wiley, 1999.

[31] B.H. Calhoun, D.C. Daly, Naveen Verma, D.F. Finchelstein, D.D. Wentzloff, A. Wang, Seong-Hwan Cho, and A. Chandrakasan, "Design considerations for ultra-low energy wireless microsensor nodes," IEEE Transactions on Computers, vol. 54, no. 6, p. 727-740, June 2005.

[32] G. Moore, "Progress in digital integrated electronics," IEEE journal of Solid-State Circuits Society Newsletter, vol. 20, no. 3, pp. 11-13, Sept. 2006.

[33] A. Raychowdhury, S. Mukhopadhyay, and K. Roy, "Modeling and estimation of leakage in sub-90nm devices," in Proceedings of 17th International Conference on VLSI Design, 2004.

[34] S. Mukhopadhyay, A. Raychowdhury, and K. Roy, "Accurate estimation of total leakage in nanometer-scale bulk CMOS circuits based on device geometry and doping profile," Transactions on Computer-Aided Design of Integrated Circuits and Systems, vol. 24, no. 3, p. 363-381, March 2005.

[35] D. W. Greve, Field Effect Devices and Aplications, London: Printice-Hall, 1998.

[36] A. Bellaouar, A. Fridi, M.I. Elmasry, and K. Itoh, "Supply voltage scalin for temperature insensitive cmos circuit operation," IEEE Transactions on Circuits and Systems II: Analog and Digital Signal Processing, vol. 45, no. 3, pp. 415-417, March 1998.

[37] M. H. Abu-Rahma, M. Anis, "A Statistical Design-Oriented Delay Variation Model Accounting for Within-Die Variations," IEEE Transactions on Computer-Aided Design of Integrated Circuits and Systems, vol. 27, no. 11, pp. 1983-1995, 2008.

[38] B. Voss and M. Glesner, "A Low Power Sinusoidal Clock," in IEEE International Symposium on Circuits and Systems, ISCAS 2001, Sydney, NSW, 2001.

[39] M. Alioto, E. Consoli, and G. Palumbo, "Physical design aware selection of energyefficient and low-energy nanometer flip-flops," in International Conference on Microelectronics (ICM), Cairo, 2010.

[40] M. Alioto, E. Consoli, and G. Palumbo, "Analysis and Comparison in the EnergyDelay-Area Domain of Nanometer CMOS Flip-Flops: Part I - Methodology and Design Strategies," IEEE Transactions on Very Large Scale Integration (TVLSI) Systems, vol. 19, no. 5, pp. 725-736, May 2011.

[41] M. Hamada, T. Terazawa, T. Higashi, S. Kitabayashi, S. Mita, Y. Watanabe, M. Ashino, H. Hara, T. Kuroda, "Flip-Flop Selection Technique for Power-Delay Tradeoff," in IEEE International Conference on Solid-State Circuits, San Francisco, CA, Feb.1999.

[42] B. Nicolick, V. Stojanovic, V. G. Oklobdzija, W. Jia, J. chiu, M. Leung, "Sense Amplifier Based Flip-Flop," in IEEE International Conference on Solid-State Circuits, San Francisco, CA, Feb. 1999. 
[43] B. Kong, S.-S. Kim, and Y.-H. Jun, "Conditional Capture Flip-Flop Technique for Statistical Power Reduction," in IEEE International Conference on Solid-State Circuits, San Francisco, CA, USA, Feb. 2000.

[44] Linfeng Li and Jianping Hu, "A Transmission Gate Flip - Flop Based on DualThreshold CMOS Techniques," in 52nd IEEE International Midwest Symposium on Circuits and Systems, Cancun, 2009.

[45] N. Weste, K. Eshraghian, Principles of CMOS Digital Design, Addison-Wesley, January 1993.

[46] L. Wei, Z. Chen, M. Johnson, K. Roy and V. De, "Design and Optimization of Low Voltage High Performance Dual Threshold CMOS Circuits," in Proceedings of IEEE International Conference on Design Automation, San Francisco, CA, USA, March 1999.

[47] S. Mutoh, S. Shigematsu, Y. Matsuya, H. Fukuda, "A 1V Multi-Threshold Voltage CMOS DSP with an Efficient Power Management Technique for Mobile Phone Application," IEEE Journal of Solid-State Circuits, vol. 30, no. 8, p. 1795-1802, 1996.

[48] John P. Uyemura, Introduction to VLSI Circuits and Systems, Wiley, 2002.

[49] V. Suzuki, K. Odagawa, T. Abe, "Clocked CMOS calculator circuitry," IEEE Journal of Solid-State Circuits, vol. 8, no. 6, pp. 462-469, 1973.

[50] S. Amarchinta, H. Kanitkar, D. Kudithipudi, "Robust and high performance subthreshold standard cell design," in 52nd IEEE International Midwest Symposium on Circuits and Systems, Cancun, 2009. 


\section{Appendix A: Body-Bias Mechanisms}

The body effect occurs due to potential difference between the source and body of a transistor [9] . It can be demonstrated as being an increase in the threshold voltage $V_{t h}$ for a NMOS transistor that occurs when the source and substrate have different voltage potentials. With this effect taken into account, the threshold voltage for a n-channel transistor is [9]:

$$
\mathrm{V}_{\mathrm{th}}=\mathrm{V}_{\mathrm{th} 0}+\gamma\left(\sqrt{\left|-2 \emptyset_{f}+V_{S B}\right|}-\sqrt{\left|2 \emptyset_{F}\right|}\right.
$$

where $V_{\text {tho }}$ is the threshold voltage without body effect $\left(\mathrm{V}_{\mathrm{SB}}=0\right), \varnothing_{F}$ is the difference between the Fermi potential of the substrate and intrinsic silicon and also factor $\gamma$ is called the body-effect constant, as shown in Equation (A-2):

$$
\gamma=\frac{\sqrt{2 q N_{A} K_{S} \varepsilon_{0}}}{C_{o x}}
$$

where $N_{A}$ is the doping concentration, $K_{S}$ is the relative permittivity of silicon, $\varepsilon_{0}$ is the permittivity of free space and Cox is the gate oxide capacitance. The body-effect constant is proportional to the doping concentration.

Body-bias rules can improve the inverse subthreshold slope $\mathrm{S}$ due to reduced shortchannel effects and reduce the junction capacitances by increasing the junction depletion widths [3]. These effects lead to faster operation and lower power consumption in a subthreshold device.

Body-bias methods have been offered as a method for decreasing threshold voltage variations. This is presented in Equation (A-3):

$$
V_{t h}=V_{t h 0}-\lambda_{d s} V_{d s}-\lambda_{b s} V_{b s}
$$


Where $V_{t h 0}$ is the threshold voltage without the body effect, $\lambda_{d s}$ is the drain induced barrier lowering the DIBL coefficient, and $\lambda_{b s}$ is the body effect coefficient. As Equation (A-3) shows, threshold voltage linearly changes $V_{d s}$ and $V_{b s}$. Figure A.1shows that the value of $\lambda$ is indicated in the linear slope of threshold voltage versus the $V_{d s}$ and $V_{b s}$ plots. In addition, $\lambda$ is independent in temperature but intercepts changes in temperature. As Table (A.1) shows, $\lambda_{d s}$ is small, so the second term of Equation (A-3) can be neglected. Hence, as $V_{b s}$ increases, threshold voltage decreases.

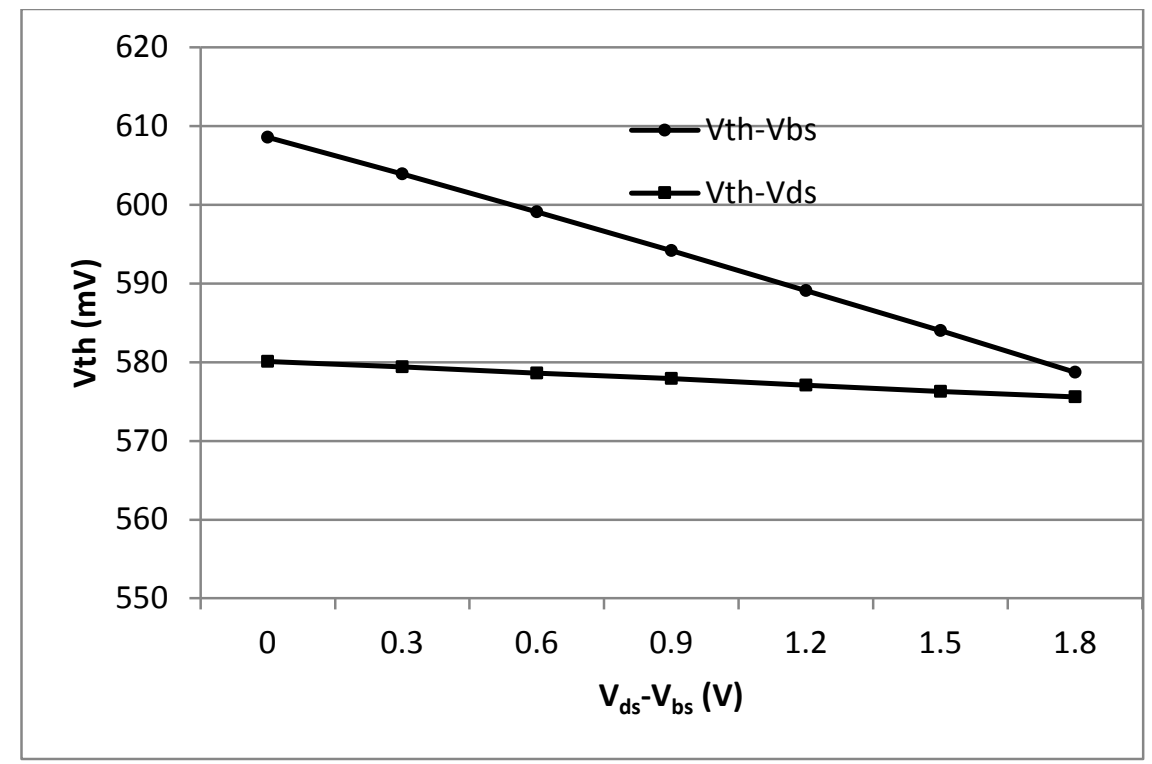

Figure A.1 $V_{t h}$ versus $V_{d s}$ and $V_{b s}\left(L=L_{\min }=60 \mathrm{~nm}, W=W_{\min }=120 \mathrm{~nm}, V_{d d}=200 \mathrm{mV}, 65 \mathrm{~nm}\right.$ process, hvt-NMOS).

Table A.1. $\lambda$ values $\left(L=L_{\min }=60 \mathrm{~nm}, W=W_{\min }=120 \mathrm{~nm}, V_{d d}=200 \mathrm{mV}, 65 \mathrm{~nm}\right.$ process, hvt-NMOS).

\begin{tabular}{|c|c|c|}
\hline & $\lambda_{d s}$ & $\lambda_{b s}$ \\
\hline NMOS-hvt & 0.01 & 0.2 \\
\hline
\end{tabular}

In this section, dynamic threshold voltage logic is presented and shows an important improvement in stability with regard to temperature and process variations. 


\section{A.1. Dynamic Threshold Voltage (DTMOS)}

As Figure A. 2 shows, sub-DTMOS logic uses transistors whose gates are connected to their substrate [10]. Threshold voltage changes as the substrate voltage changes. The characteristics of a DTMOS transistor are exactly the same as those of regular MOS transistor. Both have the same properties, such as the same OFF-current, subthreshold slope, and threshold voltage. In the on-state, however, the substrate-source voltage is forward-biased, which reduces the threshold voltage of DTMOS transistor. The decreased threshold voltage is due to the reduction of body charge.

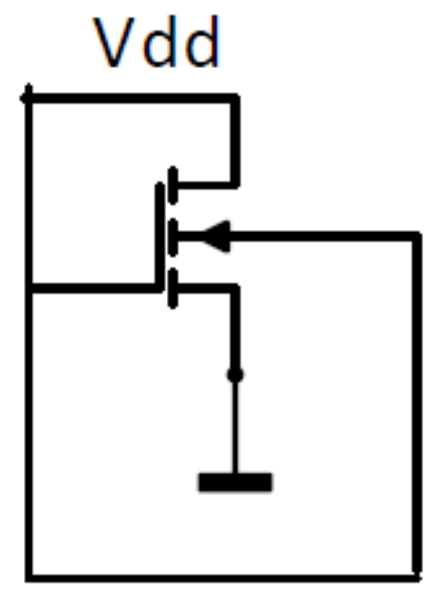

Figure A. 2 Test bench of DNMOS.

The current and threshold voltage results are shown in Table (A.2) for hvtNMOS and hvt-DTMOS in TSMC $65 \mathrm{~nm}$. This shows the performance improvement of DTMOS at the expense of higher gate leakage current. .

Table A.2. Current for hvt-NMOSwith different threshold voltages $\left(L=L_{\min }=60 \mathrm{~nm}, W=W_{\min }=\right.$ $120 \mathrm{~nm}, V_{d d}=200 \mathrm{mV}, T=27^{\circ} \mathrm{C}, 65 \mathrm{~nm}$ process).

\begin{tabular}{|l|r|c|}
\hline & hvt-NMOS & hvt-DTNMOS \\
\hline Current (pA) & 122.2 & 355.5 \\
\hline Gate Current (fA) & 1.277 & 202.6 \\
\hline Threshold Voltage (mV) & 612.8 & 570.1 \\
\hline
\end{tabular}


The frequency and current of operation of a 5-stage inverter ring oscillator shown in Figure A. 3 are recorded in Table (A.3) using hvt and hvt-DNMOS devices. As it can be seen, the frequency and current have increased by $20 \%$ and $75 \%$ respectively using a hvtDT-NMOS ring oscillator. Since current is proportional to frequency and DTMOS results in higher current, the improvement in speed was expected.

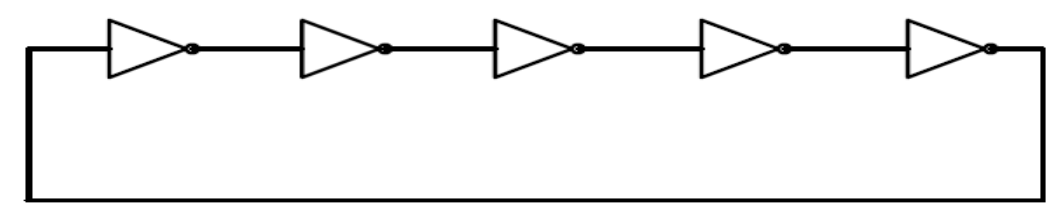

Figure A. 3. 5-Stages Inverter.

Table A.3. Current and Frequency ring oscillator for different body-bias $\left(L=L_{\min }=60 \mathrm{~nm}, W=W_{\min }\right.$ $=120 \mathrm{~nm}, V_{d d}=200 \mathrm{mV}, T=27^{\circ} \mathrm{C}, 65 \mathrm{~nm}$ process, hvt).

\begin{tabular}{|c|c|c|c|}
\hline & RO & D-RO & Increase\% \\
\hline$I$ & $17.09 \mathrm{p}$ & $30.05 \mathrm{p}$ & $75 \%$ \\
\hline$f$ & $41.1 \mathrm{k}$ & $48.64 \mathrm{k}$ & $20 \%$ \\
\hline$P$ & $5.041 \mathrm{p}$ & $8.1 \mathrm{p}$ & $48 \%$ \\
\hline
\end{tabular}

As shown in Figure A. 4, the frequency versus supply voltage is plotted for ring oscillator and dynamic ring oscillator. This plot indicates that connecting body to gate improves frequency. The increase in frequency and current is not the same as frequency depends on short circuits, load capacitance and power. The power consumption and short circuit current rise almost $48 \%, 7 \%$ respectively for DNMOS, and load capacitances changes are insignificant. Hence, dynamic and static power consumptions cause a decline in frequency.

From the current diagram in Figure A. 5, we can see that the reason for higher power dissipation is the greater amount of leakage (static) current when the inverters are 
not switching. This is because of reduction in $V_{t h}$. Thus, we learned that connecting the body to gate improves the speed but at the expense of higher static power dissipation.

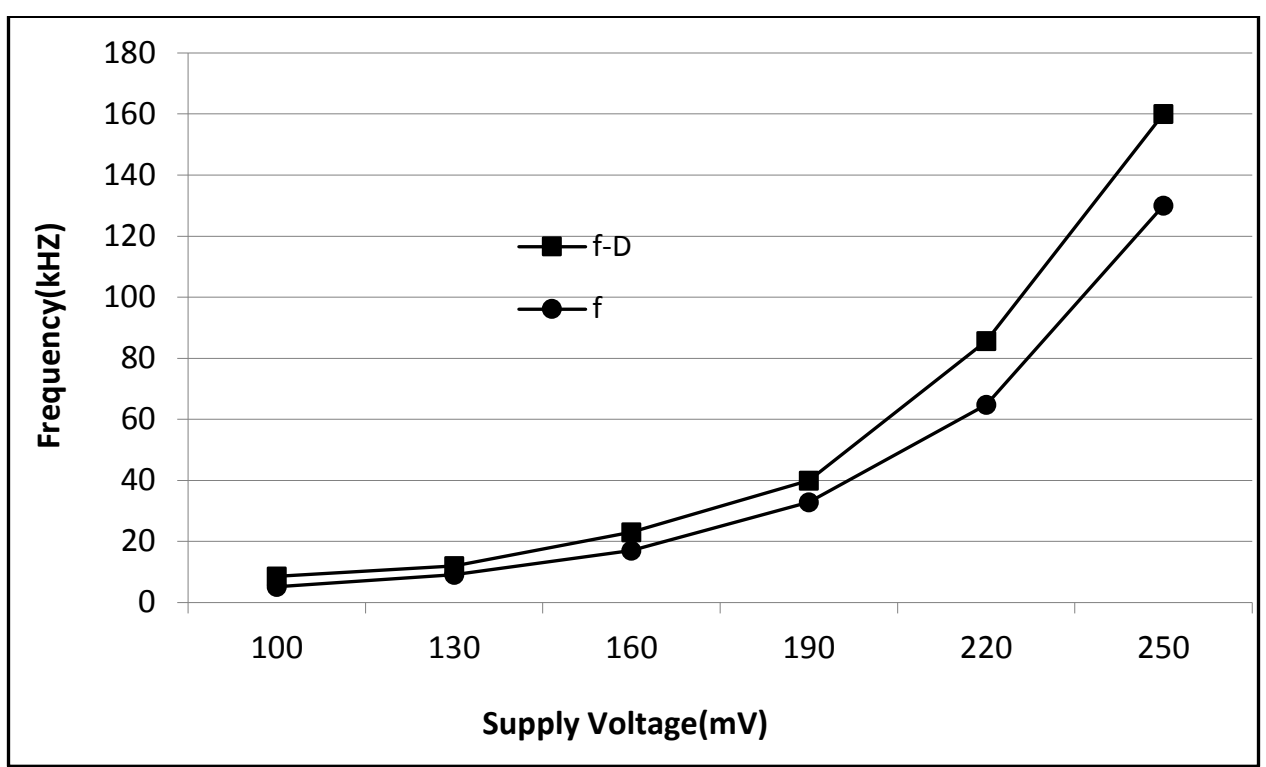

Figure A. 4. Frequency versus supply Voltage for RO and D-RO $\left(L=L_{\min }=60 \mathrm{~nm}, W=W_{\min }=\right.$ $120 \mathrm{~nm}, V_{d d}=200 \mathrm{mV}, T=27^{\circ} \mathrm{C}, 65 \mathrm{~nm}$ process, hvt).

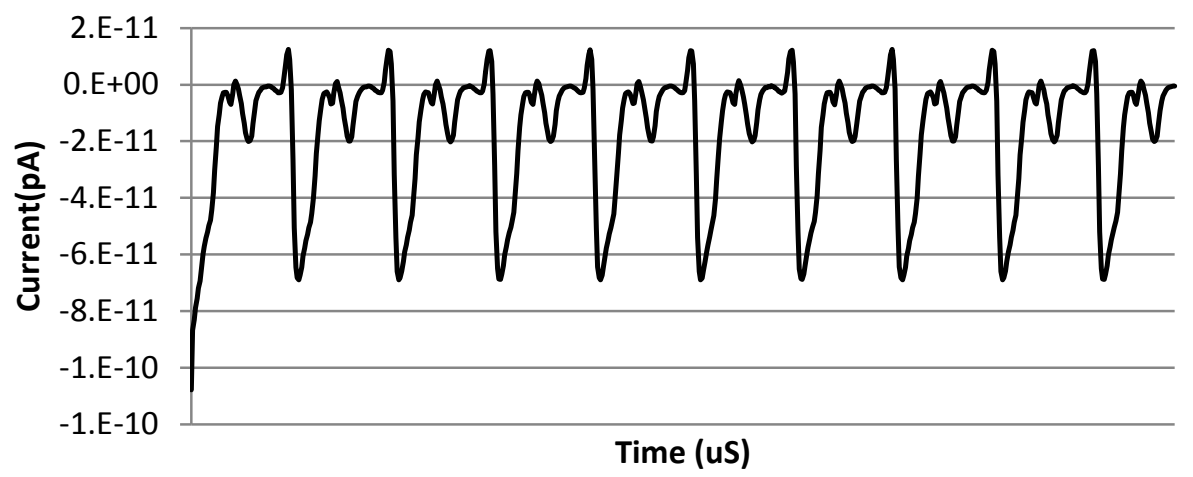

Figure A. 5. Current versus time $\left(L=L_{\min }=60 \mathrm{~nm}, W=W_{\min }=120 \mathrm{~nm}, V_{d d}=200 \mathrm{mV}, T=27^{\circ} \mathrm{C}, 65 \mathrm{~nm}\right.$ process, hvt).

Forward biasing the substrate of a MOS transistor can reduce the threshold voltage of the device. In the following section, several possible biasing mechanisms are tried to improve the frequency and robustness to process variation for an inverter. 


\section{A.2. Different Methods of Body Biasing}

The biasing mechanism shown in Figure A.6, in which the substrate of PMOS and NMOS are biased using a connection between respective gates and substrates, is termed Gate-Gate (gg) biasing [50]. The Supply-Ground (SG) biasing method for the substrate of NMOS is biased using the supply voltage, and the substrate of PMOS is biased with ground as shown in Figure A.6-b. In addition, one more biasing mechanisms involving PMOS bulk connected to the ground and NMOS to the gate (Gate-Ground or $\mathrm{gG}$ ) [50]. The results for power consumption and delay using a supply voltage of $0.2 \mathrm{~V}$ are shown in Table (A.3). As it can be seen, the Supply-Ground configuration results in the least delay, while the Zero-Bias structure yields the least power consumption.

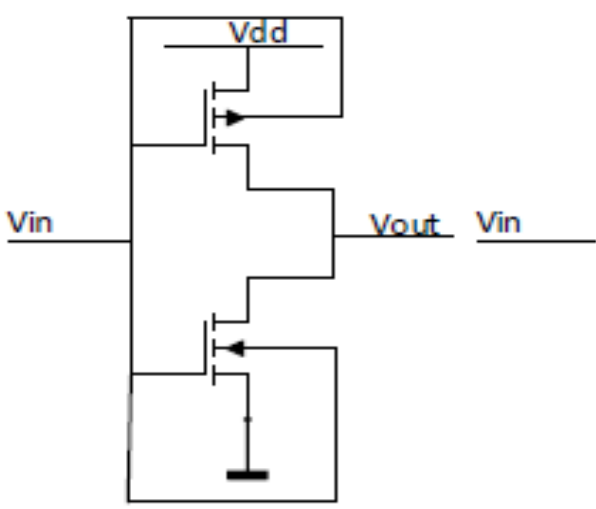

(a)

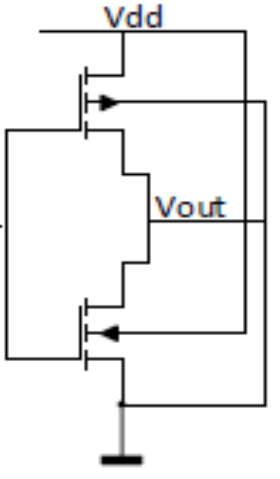

(b)

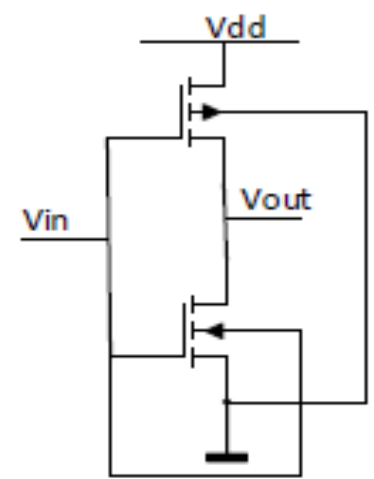

(c)

Figure A.6. Several biasing mechanisms for an inverter (a) Gate-Gate biasing (b) Supply-Ground bias-ing (c) Gate-Ground biasing.

Table A.3. Delay and Power values for inverter $\left(L=L_{\min }=60 \mathrm{~nm}, W=W_{\min }=120 \mathrm{~nm}, V_{d d}=200 \mathrm{mV}\right.$, $65 \mathrm{~nm}$ process, hvt).

\begin{tabular}{|l|l|l|}
\hline & Delay & Power \\
\hline Zero-Bias & $1.89 \mu \mathrm{s}$ & $641 \mathrm{fW}$ \\
\hline Gate-Gate & $1.3 \mu \mathrm{s}$ & $1.18 \mathrm{pW}$ \\
\hline Supply-Ground & $593.3 \mathrm{~ns}$ & $917.5 \mathrm{fW}$ \\
\hline Gate-Ground & $983.5 \mathrm{~ns}$ & $1.018 \mathrm{pW}$ \\
\hline
\end{tabular}


The body biasing for the case of Gate-Gate biasing is instantaneously applied to input signal transitions. The Supply-Ground biasing method has the greatest impact on delay when compared to other biasing schemes. This is because other structures involve substrate to gate connection. The logic states of these nodes do not necessarily provide biasing at all times with regards to input state switching. In case of Supply-Ground biasing, PMOS and NMOS are biased at all times as they are independent of input transition.

\section{A.3. Monte Carlo Simulation}

The statistical Monte Carlo simulations would be useful for in-depth examination of yield numbers. From mathematic, standard deviation $(\sigma)$ measures the amount of variation from average. Hence, lower standard deviation shows that data points are closer to the mean. In addition, $\sigma / \mu$ indicates the robustness of the circuit. Since the Monte Carlo simulation considers all corners, temperature should be set up manually.

Figure A.7 shows the relative sigma of NMOS and DNMOS at $27{ }^{\circ} \mathrm{C}, 77^{\circ} \mathrm{C}$, and -20 ${ }^{\circ} \mathrm{C}$. DNMOS is more reliable than NMOS because dynamic NMOS devices have lower relative sigma.

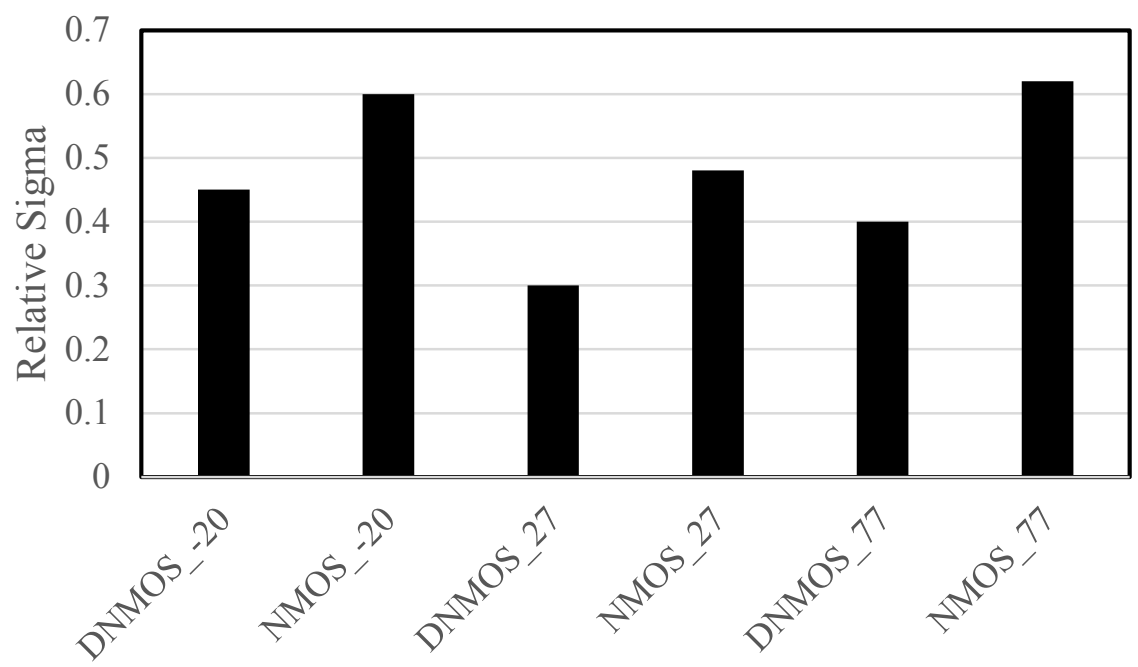

Figure A.7 Relative sigma of NMOS and DNMOS $\left(L=L_{\min }=60 \mathrm{~nm}, W=W_{\min }=120 \mathrm{~nm}, V_{d d}=200\right.$ $\mathrm{mV}, 65 \mathrm{~nm}$ process, hvt). 
Monte Carlo simulation of delay of three inverter that their body are biased as gate-gate (gg), supply-ground (SG), and gate-ground ( $\mathrm{gG})$, are considered for different process and temperature. As shown in Figure A. 8, supply-ground inverter is more reliable because has less relative sigma.

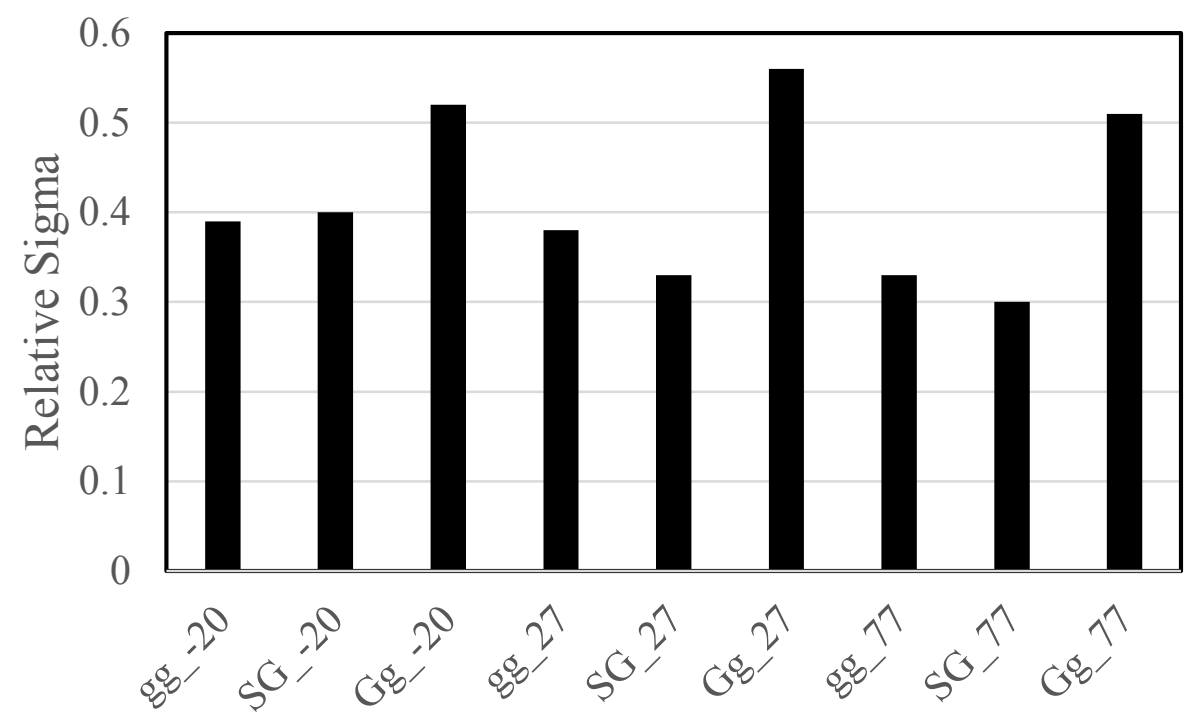

Figure A. 8. Relative Sigma of three different body-bias on inverter $\left(L=L_{\min }=60 \mathrm{~nm}, W=W_{\min }=\right.$ $120 \mathrm{~nm}, V_{d d}=200 \mathrm{mV}, 65 \mathrm{~nm}$ process, hvt).

\section{A.4. Summary}

In this appendix, we presented that dynamic threshold voltage transistors have attained improved reliability. Furthermore, three different body-bias methods have been applied to an inverter and compared for reliability using Monte Carlo simulations. In appendix B, the layout of TG flip-flop is implemented and compared to schematic. 


\section{Appendix B: Layout of Transmission Gate Flip- Flop}

The layout of designs is required for realistic simulations in the simulation environment.

By simulating on the schematic, parameters depending on the physical layout of the design cannot be modelled accurately. For example, interconnect capacitance and parasitic effects depend on length of wires and other selections done in the physical layout. In this section, the extraction of TG flip-flop schematic are considered and compared to schematic simulation results. The flip-flop layout is shown in Figure B.1.The area of layout is 6.6 $\mu \mathrm{m} \times 4.36 \mu \mathrm{m}$.

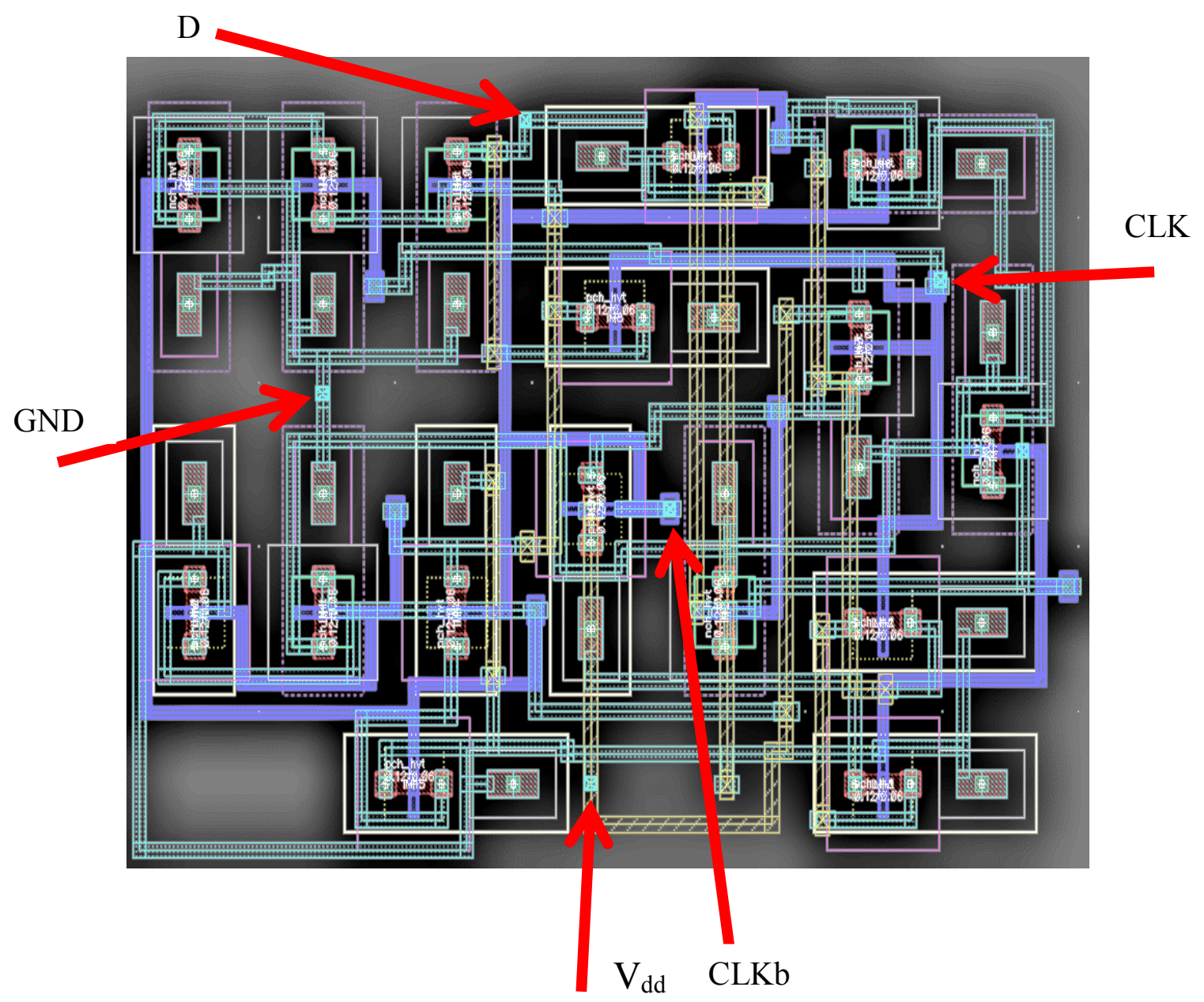

Figure B.1.Transmission gate flip-flop layout. 


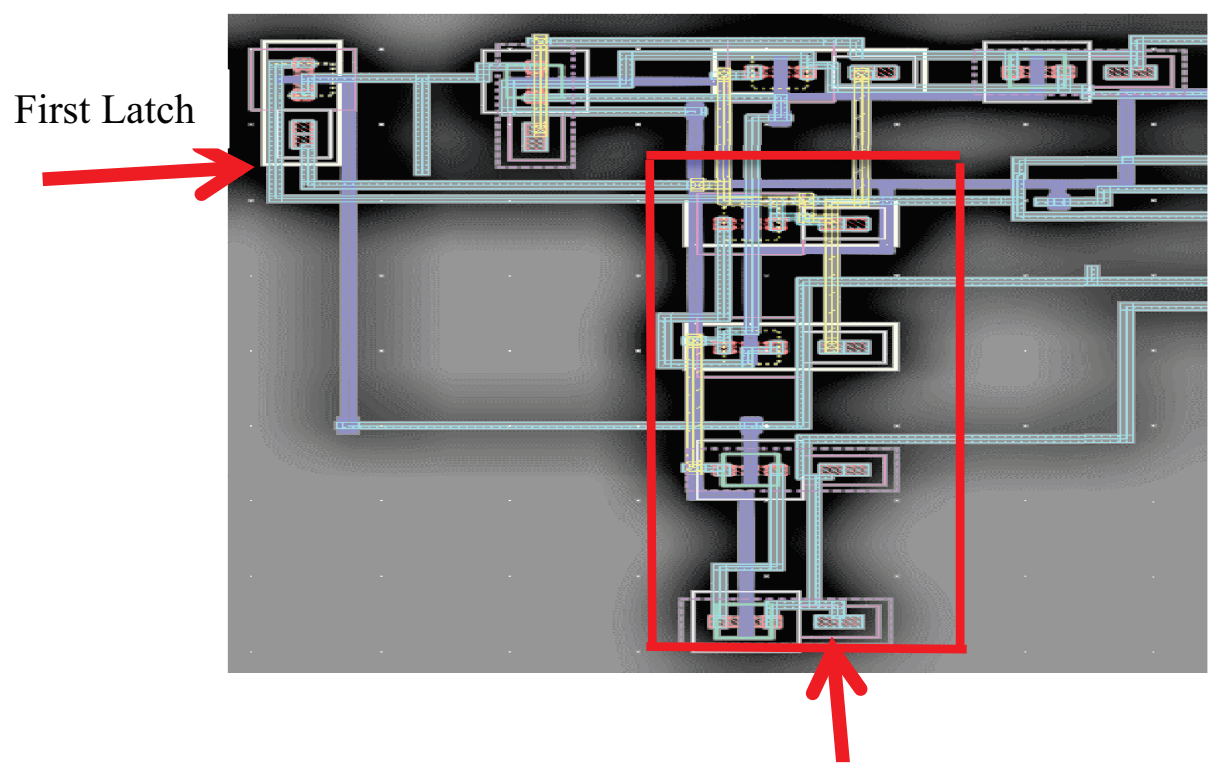

TS-Inverter

Figure B.2. Tri-State inverter in first latch layout.

Minimum size flip-flop is implemented in the layout. We used two different metals because of interconnection metal, and poly for gate connection. Tri-State inverter in feedback is shown in Figure B.2. This inverter has smaller delay and more reliability as explained in section 5.1.4.

\section{B.1 Parasitic Extraction}

By running the extraction tools, parasitic data are extracted for the different nets in the layout as is shown in Table (B.1). 
Table B.1. Layout parasitic for test circuit.

\begin{tabular}{|l|l|l|l|l|}
\hline Net & Count & C Total(F) & CC Total(F) & C+CC Total (F) \\
\hline CLK & 39 & $1.38 \mathrm{E}-15$ & $9.06 \mathrm{E}-16$ & $2.29 \mathrm{E}-15$ \\
\hline D & 18 & $2.06 \mathrm{E}-16$ & $2.32 \mathrm{E}-16$ & $4.3 \mathrm{E}-16$ \\
\hline CLKb & 58 & $7.12 \mathrm{E}-16$ & $5.25 \mathrm{E}-16$ & $1.23 \mathrm{E}-15$ \\
\hline Vdd & 106 & $1.39 \mathrm{E}-15$ & $1.90 \mathrm{E}-15$ & $3.25 \mathrm{E}-15$ \\
\hline GND & 111 & $1.62 \mathrm{E}-15$ & $1.21 \mathrm{E}-15$ & $2.84 \mathrm{E}-15$ \\
\hline Q & 46 & $1.28 \mathrm{E}-15$ & $9.65 \mathrm{E}-15$ & $2.25 \mathrm{E}-15$ \\
\hline
\end{tabular}

Figure B. 3 compares the schematic and layout total power consumption as the supply voltage changes. The results show that the total power consumption is close for the schematic and layout at $100 \mathrm{mV}$. However, as supply voltage increases, the layout consumes more power consumption compared to schematic view. At lower supply voltages, the dominating power is static. Meanwhile, as the supply voltage increases, the dynamic power consumption begins to govern. Thus, the increase in parasitic output capacitance causes the layout view to have higher total power consumption. The power consumption can increase up to $53 \%$ due to layout parasitic as shown in the plot. 


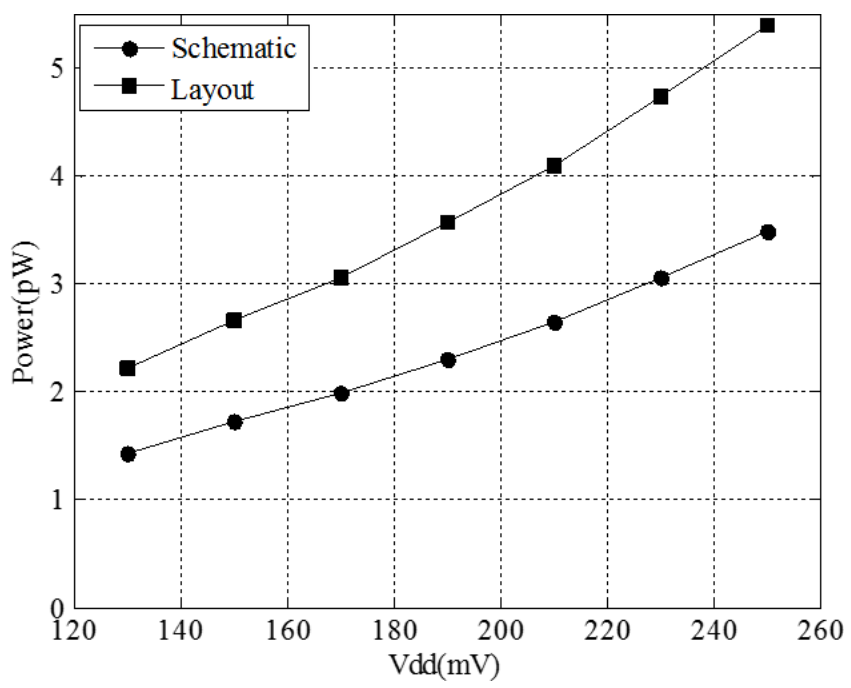

Figure B.3. Total power versus $V_{d d}\left(L=L_{\min }=60 \mathrm{~nm}, W=W_{\min }=120 \mathrm{~nm}, V_{d d}=200 \mathrm{mV}, T=27^{\circ} \mathrm{C}, 65\right.$ nm process).

The layout has higher propagation delay because of capacitance, as shown Table (B.1). The increase in delay is $38 \%$.

Table B.1. Rising and falling delay of layout and schematics $\left(L=L_{\min }=60 \mathrm{~nm}, W=W_{\min }=120 \mathrm{~nm}, V_{d d}\right.$ $=200 \mathrm{mV}, 65 \mathrm{~nm}$ process).

\begin{tabular}{|l|r|c|}
\hline & Rising-Delay & Falling-Delay \\
\hline TG-Schematic & $10.7 \mathrm{u}$ & $11.89 \mathrm{u}$ \\
\hline TG-Layout & $14.7 \mathrm{u}$ & $16.3 \mathrm{u}$ \\
\hline
\end{tabular}

The relative sigma is highest for both schematic and layout at $-20{ }^{\circ} \mathrm{C}$, in Figure B.4. 


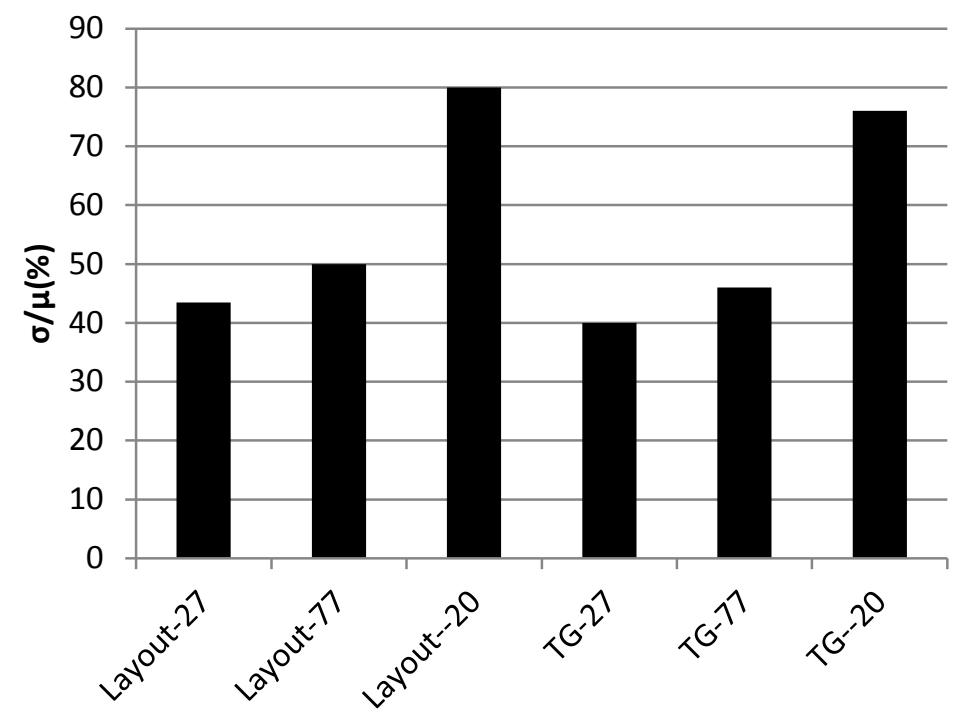

Figure B.4. Relative Sigma of schematics and layout $\left(L=L_{\min }=60 \mathrm{~nm}, W=W_{\min }=120 \mathrm{~nm}, V_{d d}=200\right.$ $\mathrm{mV}, 65 \mathrm{~nm}$ process).

As shown in Figure (B.4), the schematic view is more reliable than layout as transistor parameters and their variations are modelled more precisely using layout.

\section{B.2. Summary}

In this appendix, we have implemented the transmission gate flip-flop and compared post-layout to schematic simulations. Additional parasitic cause an increase in power and delay while decreasing reliability for the flip-flop after layout. 